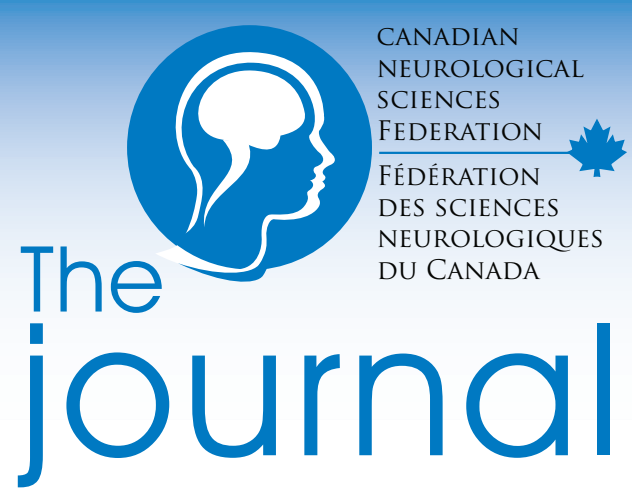

Canadian Journal of Neurological Sciences

Volume 40 Number 3 (Supplement 1) May 2013

\title{
48th Annual Congress of the \\ Canadian Neurological Sciences Federation
}

Montreal, Quebec, Canada

48e congrès annuel de la

Fédération des sciences

neurologiques du Canada

\section{ABSTRACTS / RÉSUMÉS}

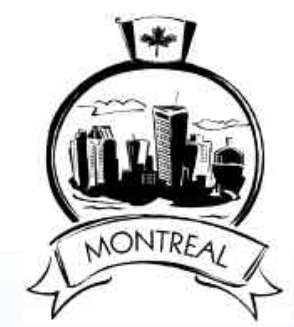




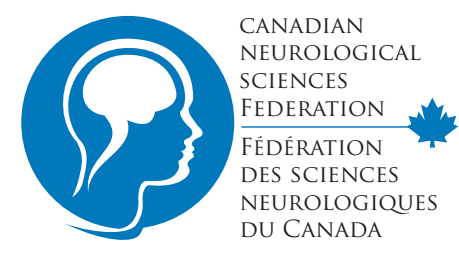

Congress Agenda as of March 21, 2013

\section{Canadian Neurological Sciences Federation Annual Congress June 12-14, 2013 - Fairmont Queen Elizabeth Hotel, Montreal, Quebec \\ Pre-Congress June 11, 2013 *SIG - Special Interest Groups}

8:30 am - 5:00 pm $6: 00 \mathrm{pm}-8: 00 \mathrm{pm}$ $6: 00 \mathrm{pm}-8: 00 \mathrm{pm}$ 6:00 pm - 8:00 pm $6: 00 \mathrm{pm}-8: 00 \mathrm{pm}$ $6: 00 \mathrm{pm}-8: 00 \mathrm{pm}$

9:00 am - 5:00 pm 9:00 am - 5:00 pm 9:00 am - 12:00 pm 9:00 am - 12:00 pm 9:00 am - 12:00 pm 12:15 pm - 1:45 pm

12:15 pm - 1:45 pm 2:00 pm - 5:00 pm 2:00 pm - 5:00 pm 2:00 pm - 5:00 pm 5:00 pm - 7:00 pm

8:30 am - 11:00 am 11:15 am - 12:15 pm 11:15 am - 12:15 pm $11: 15 \mathrm{am}-12: 15 \mathrm{pm}$ 12:15 pm - 1:45 pm

2:00 pm - 5:00 pm 2:00 pm - 5:00 pm 2:00 pm - 5:00 pm 2:00 pm - 5:00 pm 2:00 pm - 5:00 pm 5:00 pm - 6:30 pm

8:00 am - 11:00 am 11:15 am - 1:00 pm 1:00 pm - 2:15 pm 2:15 pm - 5:15 pm 2:15 pm - 5:15 pm 2:15 pm - 5:15 pm 2:15 pm - 5:15 pm 2:15 pm - 5:15 pm
Tuesday, June 11, 2013

Epilepsy: Optimizing Patient Care, from Diagnosis to Management Neurocritical Care-Brain Death SIG

Movement Disorders SIG

Epilepsy Video SIG

Headache: Migraine \& Friends SIG

Neuromuscular SIG

Wednesday, June 12, 2013

Neurosurgery Residents: Emergency Neurosurgery

Neurology Residents: Movement Disorders \& Parkinson's Disease Stroke

Hot Topics in Child Neurology

Minimally Invasive Cranial Neurosurgery

A Practical Financial Road Map for Neurological/

Neurosurgery Residents

Treatable Myopathies State of the Art in Diagnosis \& Management Headache

Neuromuscular

Neurocritical Care

Exhibitors' Reception

Thursday, June 13, 2013

Grand Plenary

Child Neurology Day: CACN Chair's Select Abstract

CNSS Chair's Select Abstract

CNS/ CSCN Chair's Select Abstract

Applying the Canadian Guidelines on Parkinson's

Disease to our Practice

Canadian Neurosurgical Innovations \& Discoveries

Child Neurology Day

Epilepsy: From Diagnosis to Intervention

MASS: Minimal Access Spine Surgery

Promises of Stem Cells in the Neurosciences

Digital Poster Author Standby

Max Findlay, Roberto Diaz

Pierre J. Blanchet, Nailyn Rasool, Serena Orr Alex Poppe, Sylvain Lanthier

Asif Doja

Kesh Reddy

Michele Seaton-Gascon, Serena Orr

Tahseen Mozaffer

Sian Spacey

Mike Nicolle, Kristine Chapman

Jeanne Teitelbaum, Draga Jichici

Michelle Demos, Craig Campbell

Brian Toyota, Ramesh Sahjpaul Michelle Demos, Craig Campbell Jorge Burneo

Eric Massicotte

Peter Dirks
Platform Sessions

Grand Rounds

Digital Poster Author Standby

Difficult Cases and Controversies in Neurosurgery (Cranial/Spinal) Multiple Sclerosis

Neurovascular \& Interventional Neuroradiology

Genetics of Neurologic \& Neurodegenerative Syndromes

Neuro-Opthalmology
Joseph Megyesi

Paul Giacomini, Catherine Larochelle Gary Redekop

Matthew Farrer

Jason Barton 


\title{
48th Annual Congress of the
}

\author{
Canadian Neurological Sciences Federation
}

\section{MONTREAL • QUEBEC ・ JUNE 11-14, 2013}

CNSF Executive Committee

J. Max Findlay, CNSF President

Jeanne Teitlebaum, CNSF Vice President

Chris Wallace, CNSF Vice President

Garth Bray, CNSF Vice President

Dan Morin, CNSF CEO

Scientific Program Committee \& Professional Development Committee Robert Loch Macdonald, CNSS, Chair SPC Draga Jichici, CNS, Vice Chair SPC

Ron Pokrupa, CNSS, Chair PDC

Bev Prieur, CACN/CNS, Vice Chair PDC J. Max Findlay, CNSF President SPC

Jeanne Teitlebaum, CNSF Vice President

Chris Wallace, CNSF Vice President PDC

Garth Bray, CNSF Vice President PDC

Lawrence Korngut, SPC

Michelle Demos, SPC

Eric Massicotte, SPC

Simon Walling, SPC

Seyed Mirsattari, SPC

Craig Campbell, SPC

Danielle Andrade, SPC

James Perry, SPC

Michael Hill, SPC

Nailyn Rasool, CNS Residents Rep

Serena Orr, CACN Residents Rep

Roberto Diaz, CNSS Residents Rep

Rudolf Arts, PDC

Roger McKelvey, PDC

Asif Doja, PDC

Joe Megyesi, PDC

Dan Morin, CNSF CEO

Lisa Bicek, CNSF

Canadian Neurological Sciences Federation

709 - 7015 Macleod Trail SW,

Calgary, Alberta, T2H 2K6, Canada

Tel: (403) 229-9544 - Fax: (403) 229-1661

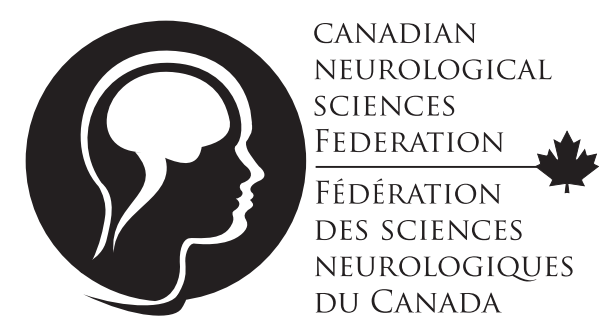

\section{ABSTRACTS AND PROGRAM}

\author{
The Canadian Journal of \\ Neurological Sciences \\ and \\ The Canadian Neurological \\ Sciences Federation
}

do not assume any responsibility or liability for any errors in the abstracts. 


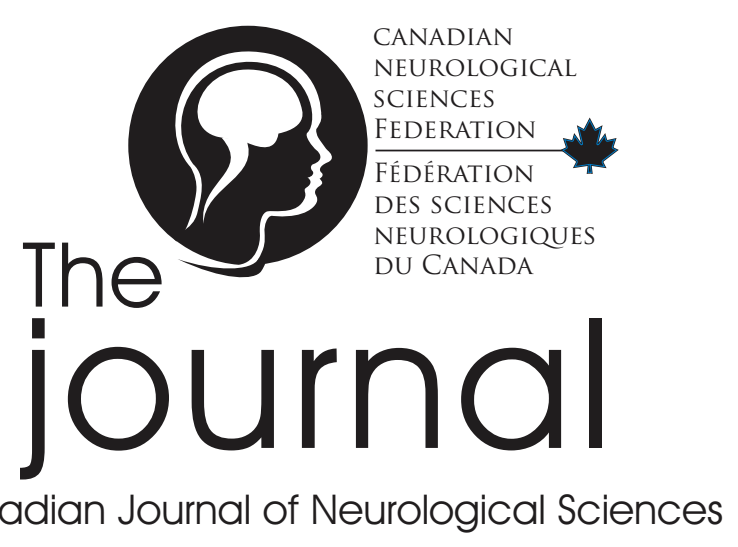

\section{Volume $40, /$ Number 3 I Supplement 1 / May 2013}

Editor-in-Chief/Rédacteur en chef

G. Bryan Young LONDON, ON

\section{Associate Editors/Rédacteurs associés}

J. Max Findlay EDMONTON, AB

Mark Sadler HALIFAX, NS

Mike Poulter LONDON, ON

Serge Gauthier VERDUN, QC

Robert Hammond LONDON, ON

Mary Connolly vancouver, BC

\section{Past Editors/Anciens rédacteurs en chef}

Douglas W. Zochodne CALGARY, AB

James A. Sharpe TORONTO, ON

Robert G. Lee CALGARY, AB

Robert T. Ross WINNIPEG, MB

(Emeritus Editor, Founding Editor)

\section{Editorial Board/Comité éditorial}

Jorge Burneo LONDON, ON

Richard Desbiens QUEBEC CITY, QC

David Fortin SHERBROOKE, QC

Mark Hamilton CALGARY, AB

Hans-Peter Hartung DUSSELDORF, GERMANY

Michael Hill CALGARY, AB

Alan C. Jackson WINNIPEG, MB

Daniel Keene OTTAWA, ON

James Perry TORONTO, ON

Oksana Suchowersky EDMONTON, AB

Brian Toyota VANCOUVER, BC

Brian Weinshenker ROCHESTER, MN, USA

Samuel Wiebe CALGARY, AB

Elaine Wirrell ROCHESTER, MN, USA

\section{SECTION EDITORS/CONSEIL DE RÉDACTION}

Neuroimaging Highlight/Neuroimagerie

David Pelz LONDON, ON

Neuropathological Conference/Conférence sur la neuropathologie

Robert Hammond LONDON, ON

Book Review/Critiques de livres

Reflections/Reflets

Andrew Kirk SASKATOON, SK

\section{Critically Appraised Topic Summaries} (CATS)

Jorge Burneo LONDON, ON

Mary Jenkins LONDON, ON
Editorial Review Board/Comité de lecture

Donald Brunet KINGSTON, ON

Jodie Burton CALGARY, AB

Lionel Carmant MONTREAL, QC

Colin Chalk MONTREAL, QC

K. Ming Chan EDMOnTON, AB

Robert Chen TORONTO, ON

Joseph Dooley HALIFAX, NS

Paolo Federico CALGARY, AB

Daryl Fourney SASKATOON, SK

Hannah Glass SAN FRANCISCO, CA, USA

Alan Goodridge ST. JOHN's, NL

Ian Grant HALIFAX, NS

Alan Guberman OTTAWA, ON

John Hurlbert CALGARY, AB

Manouchehr Javidan VANCOUVER, BC

Patrick McDonald WINNIPEG, MB

Martin McKeown vanCOUvER, BC

Joseph Megyesi LONDON, ON

Vivek Mehta EDMONTON, AB

Steven Miller TORONTO, ON

Neelan Pillay CALGARY, AB

Christopher Power EDMONTON, AB

Alex Rajput SASKATOON, SK

Jean Raymond MONTREAL, QC

Gary Redekop VANCOUVER, BC

Harvey Sarnat CALGARY, AB

John Stewart VANCOUVER, BC

Jeanne Teitelbaum MONTREAL, QC

Eve Tsai OTTAWA, ON

Shannon Venance LONDON, ON

Matt Wheatley EDMONTON, AB

Jerome Yager EDMONTON, AB

Journal Staff/Effectif du journal

Dan Morin CALGARY, AB Chief Executive Officer

Cindy Leschyshyn CALGARY, AB

Editorial Coordinator

Maggie McCallion CALGARY, AB Designer/Production Coordinator

\section{Advertising representative/}

Représentant de publicité

Dan Morin, Chief Executive Officer

Tel (403) 229-9544 Fax (403) 229-1661

E-mail: dan-morin@cnsfederation.org

\section{Printer/Imprimeur}

Unicom Graphics, 4501 Manitoba Road SE Calgary, Alberta T2G 4B9
The official journal of: / La revue officielle de :

$$
\begin{aligned}
& \text { The Canadian Neurological Society } \\
& \text { La Société Canadienne de Neurologie } \\
& \text { The Canadian Neurosurgical Society }
\end{aligned}
$$
La Société Canadienne de Neurochirurgie

The Canadian Society of Clinical Neurophysiologists La Société Canadienne de Neurophysiologie Clinique

The Canadian Association of Child Neurology

L'Association Canadienne de Neurologie Pédiatrique

The permanent secretariat for the four societies and the Canadian Neurological Sciences Federation is at: Le secrétariat des quatre associations et de la Fédération des sciences neurologiques du Canada est situe en permanence à :

7015 Macleod Trail SW, Suite 709

Calgary, Alberta, Canada T2H 2K6

CNSF (403) 229-9544 / CJNS (403) 229-9575 Fax (403) 229-1661

The Canadian Journal of Neurological Sciences is published bimonthly. The annual subscription rate for Individuals (print and online) are: C $\$ 178.00$ (Canada), C\$208.00 (US), C $\$ 292.00$ (International). Subscription rates for Institutions (print and online) are $\mathrm{C} \$ 198.00$ (Canada), C $\$ 228.00$ (US), C $\$ 312.00$ (International). "Online Only"- C $\$ 160.00$ (Individual), C \$180.00 (Institutional). See www.cjns.org for full details including taxes. Single copies $C \$ 35.00$ each plus $\mathrm{C} \$ 25.00$ shipping and handling. E-mail: journal@cjns.org. COPYRIGHT( 2013 by THE CANADIAN JOURNAL OF NEUROLOGICAL SCIENCES INC. All rights reserved. No part of this journal may be reproduced in any form without the prior permission of The Canadian Journal of Neurological Sciences. Postage paid at Calgary, Alberta.

Le Journal Canadien des Sciences Neurologiques est publié tous les deux mois. Voici les prix d'abonnement pour les personnes (imprimé et en ligne) : 178,00 \$ CA (Canada), 208,00 \$CA (É.-U.), 292,00 \$ CA (international). Voici les prix d'abonnement pour les institutions (imprimé et en ligne) : 198,00 \$ CA (Canada), 228,00 \$ CA (É.-U.), 312,00 \$ CA (international). « En ligne seulement »160,00 \$CA (personnes), 180,00 \$CA (institutions). Visiter www.cjns.org pour tous les détails incluant les taxes. Exemplaires uniques : 35,00 \$ CA l'unité, plus 25,00 \$ CA en frais de port et de manutention.Courriel : journal@cjns.org. COPYRIGHT (C) 2013 du THE CANADIAN JOURNAL OF NEUROLOGICAL SCIENCES INC. Tous droits réservés. Aucune partie de ce journal ne peut être reproduite sous quelque forme que ce soit sans la permission préalable du Journal Canadien des Sciences Neurologiques. Frais de port payés à Calgary, en Alberta.

This journal is indexed by / Cette revue est indexée par Adis International, ArticleFirst, BIOBASE, BiolAb, BiolSci, BIOSIS Prev, Centre National de la Recherche Scientifique, CSA, CurAb, CurCont, De Gruyter Saur, E-psyche, EBSCO, Elsevier, EMBASE, FRANCIS, IBZ, Internationale Bibliographie der Rezensionen Geistes-und Sozialwissenschafticher Literatur, MEDLINE, MetaPress, National Library of Medicine, OCLC, $P E \& O N$, Personal Alert, PsycFIRST, PsycINFO, PubMed, Reac, RefZh, SCI, SCOPUS, Thomson Reuters, TOCPrem, VINITI RAN, Web of Science.

ISSN $0317-1671$ 


\title{
48th Annual Congress of the
}

\section{Canadian Neurological Sciences Federation}

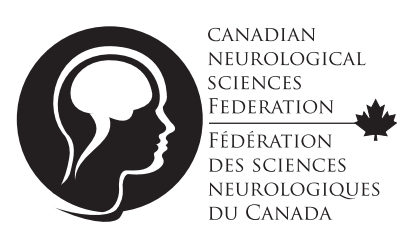

\author{
MONTREAL, QC JUNE 12-14, 2013
}

\section{Abstracts \\ Society Prize Presentations}

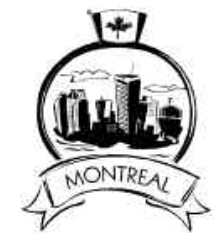

Canadian Neurological Society - André Barbeau Memorial Prize - S5

Canadian Society of Clinical Neurophysiologists - Herbert Jasper Prize - S5

Canadian Neurosurgical Society - K.G. McKenzie Prize in Clinical Neuroscience Research - S5

Canadian Neurosurgical Society - K.G. McKenzie Prize in Clinical Neuroscience Research - S6

Canadian Association of Child Neurology - The Presidents Prize - S6

\section{Platform Presentations}

\section{Thursday, June 13, 2013}

A. CNS/CSCN Chair's Select Abstract Presentations A.01 to A.04 .S7

B. CNSS Chair's Select Abstract Presentations B.01 to B.04 . .S8

C. CACN Chair's Select Abstract Presentations C.01 to C.04
Friday, June 14, 2012

D. Stroke - D.01 to D.11 ................... 10

E. Spine $-E .01$ to $E .11 \ldots \ldots \ldots \ldots \ldots \ldots \ldots$

F. Trauma \& General Neurosurgery - F.01 to F.11 . .S 16

G. Neuro-oncology \& Education - G.01 to G.11 ... .S 19

H. Epilepsy - H.01 to H.11

I. Aneurysms \& Subarachnoid Hemorrhage - I.01 to I.11 S 25

J. Pediatrics - J.01 to J.11

\section{Poster Presentations}

\section{Thursday, June 13th and Friday, June 14th, 2012}

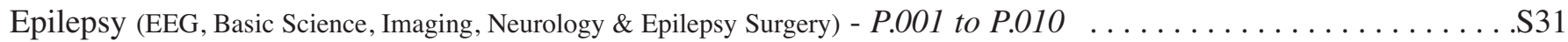

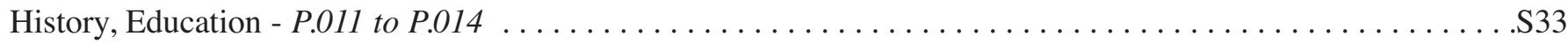

Movement Disorders (Basic Science, Neurology, Imaging \& Functional Neurosurgery) - P.015 to P.022 _ . . . . . . . . .S34

Neuro-oncology (Medical \& Radiation Oncology, Imaging, Tumour Surgery, Basic Science) - P.023 to P.033 … . . . . .S35

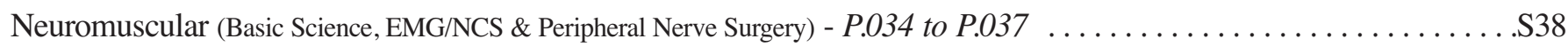

Stroke (Vascular Neurology, Imaging, Basic Science, Neurovascular/Endovascular Surgery) - P.038 to P.057 . . . . . . . . . .S39

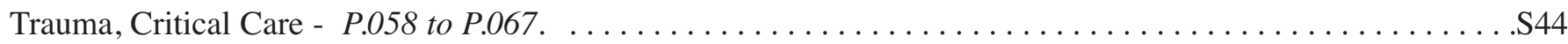

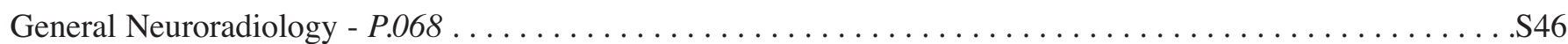

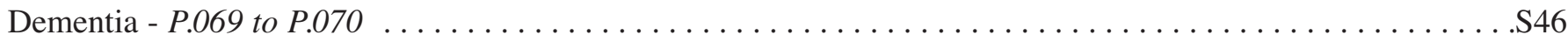

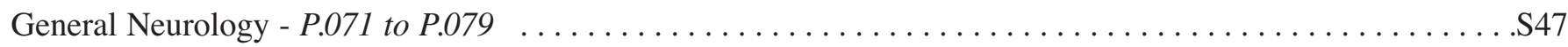

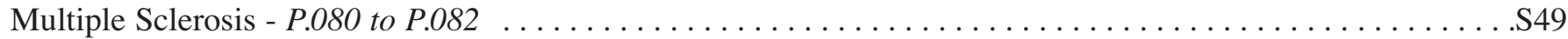

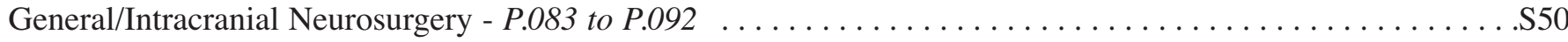

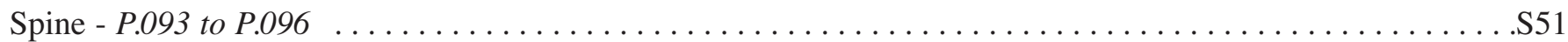

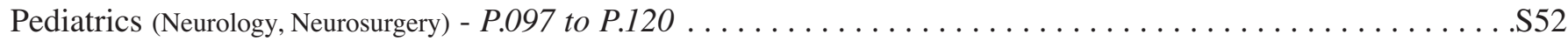

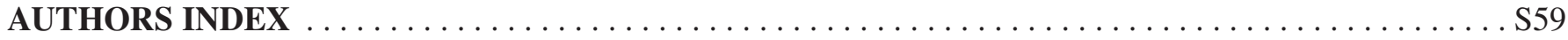

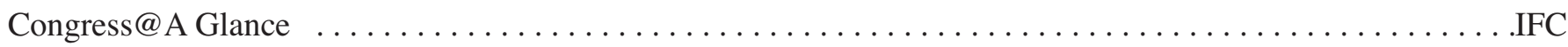




\section{CNSF Congress in Beautiful Banff Alberta}

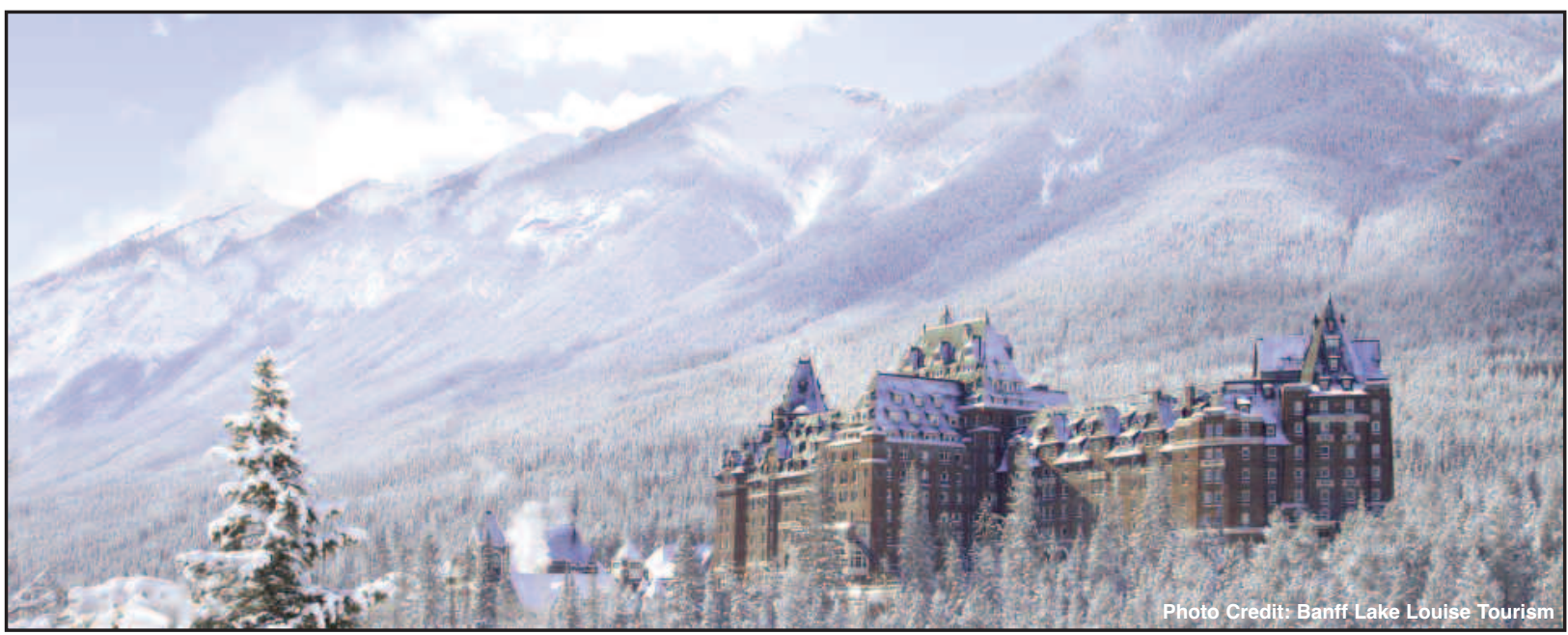

Once again we have secured an outstanding hotel property as our Congress venue and host hotel. We will be at the Fairmont Banff Springs Hotel - June 3 to June 6, 2014!

Few hotels in the world rival the majesty, hospitality and grandeur of The Fairmont Banff Springs resort, located in the heart of Banff National Park, a UNESCO World Heritage Site. This hotel was styled after a Scottish Baronial Castle and when it opened in 1888, it marked the birthplace of tourism in the Canadian Rockies.

The Fairmont Banff Springs hotel, a National Historical Site of Canada, provides unparalleled options and unique experiences to guests, from inclusive onsite getaways to days of adventure exploring the Rockies.

For the convenience of our Congress delegates, the CNSF has reserved transportation from the Calgary International Airport directly to Banff, through Brewster Corporate Event and Management. The fee is $\$ 40.50$ per person, about the same as a taxi fare from the airport to a downtown Calgary Hotel. Enjoy the ride and Enjoy the scenery.

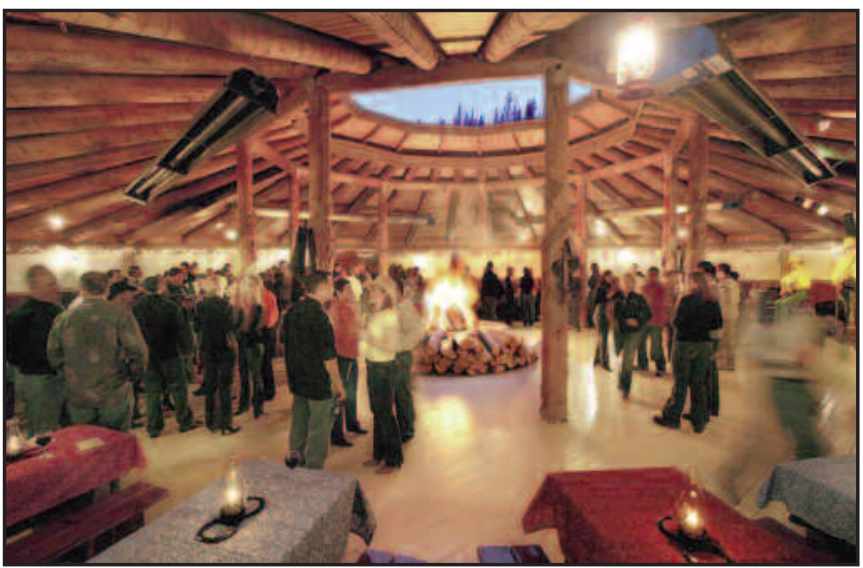

During your stay in our Rocky Mountains, we invite you to join your colleagues for a truly Western barbeque experience amid the majestic mountain peaks.

Just minutes from Banff at Brewster's Mountainview location we will gather 'round the indoor central bonfire for an evening of entertainment and feasting.

Transportation to and from the venue is provided.

Then, looking forward to 2015! The CNSF 50th Annual Congress!

Hope to see you all at the Fairmont Royal York Hotel in Toronto, June 9 to June 12, 2015. 


\section{Society Prize Papers}

\section{ANDré Barbeau Memorial Prize}

\section{Canadian Neurological Society}

\section{A.03}

Titin founder mutation is a common cause of myofibrillar myopathy with early respiratory failure

G Pfeffer (Vancouver)* R Barresi (Newcastle) IJ Wilson (Newcastle) SA Hardy (Newcastle) H Griffin (Newcastle) J Hudson (Newcastle) HR Elliott (Newcastle) AV Ramesh (Newcastle) A Radunovic (London) J Winer (Birmingham) S Vaidya (London) A Raman (Hull) $M$ Busby (Leeds) ME Farrugia (Glasgow) A Ming (Hull) C Everett (London) HC Emsley (Manchester) R Horvath (Newcastle) V Straub (Newcastle) H Lochmuller (Newcastle) PF Chinnery (Newcastle) A Sarkozy (Newcastle)

Objective: Titin gene (TTN) mutations have been described in 8 families with hereditary myopathy with early respiratory failure (HMERF). Some of the original patients had features resembling myofibrillar myopathy (MFM), arguing that TTN mutations could be a cause of MFM. Methods: We studied 127 undiagnosed patients with clinical presentation compatible with MFM. Sanger sequencing for the two previously described TTN mutations in HMERF (p.C30071R in the 119th fibronectin-3 (FN3) domain, and p.R32450W in the kinase domain) was performed in all patients. Results: We identified 5 new families with the p.C30071R mutation who were clinically similar to previously reported cases, and muscle pathology demonstrated diagnostic features of MFM. Two further families had novel variants in the 119th FN3 domain (p.P30091L and p.N30145K). No patients had mutations at p.32450. Conclusions: Mutations in TTN are a cause of MFM, and titinopathy is more common than previously thought. The finding of the p.C30071R mutation in $3.9 \%$ of our study population is likely due to a British founder effect. The occurrence of novel FN3 domain variants, although still of uncertain pathogenicity, suggests that other mutations in this domain may cause MFM, and that the disease is likely globally distributed. We suggest that HMERF due to mutations in the TTN gene be nosologically classified as MFMtitinopathy.

\section{Herbert Jasper Prize}

\section{Canadian Society of Clinical NEUROPHYSIOLOGISTS \\ H.01}

Using StatNet EEGs in the emergency department shortens diagnosis delay

F Moien Afshari (Saskatoon)* JA Edlow (Boston) HT Susan (Boston) M Barnard (Boston) TD Pang (Boston)

Background: Delays in diagnosing non-convulsive status epilepticus increases emergency department (ED) mortality. The conventional EEG set up is time consuming even by well-trained technologists. The StatNet electrode can be applied after minimal training. Methods: Hypothesis is that StatNet EEG decreases EEG recording and interpretation delay and facilitates patients' disposition from the ED. We compared several parameters between StatNet EEG with control and historical control (HC) using one-way ANOVA. Results: EEG set up was shorter in the StatNet $(34 \pm 4$, $\mathrm{N}=20)$ vs. control $(25 \pm 3.3, \mathrm{~N}=24, \mathrm{p}<0.05)$. The delay from EEG order to completion was the shortest in the StatNet group (86 \pm 9.3 , $\mathrm{N}=25)$ vs. control group $(170 \pm 25, \mathrm{~N}=25)$ vs. $\mathrm{HCs}(225 \pm 46, \mathrm{~N}=23$, $\mathrm{p}<0.01)$. Time from EEG completion to interpretation was shorter for StatNet $(39 \pm 7, \mathrm{~N}=25, \mathrm{p}<0.001)$ and control $(59 \pm 5, \mathrm{~N}=21$, $\mathrm{p}<0.01)$ groups vs. HCs $(158 \pm 48, \mathrm{~N}=7, \mathrm{p}<0.01)$. Finally, time from EEG order to ED disposition was shorter in the StatNet $(185 \pm 19$, $\mathrm{N}=20, \mathrm{p}<0.001)$ and control $(180 \pm 19, \mathrm{~N}=15, \mathrm{p}<0.01)$ vs. HCs $(374$ $\pm 37, \mathrm{~N}=19)$. Conclusions: We showed that using StatNet EEG in $\mathrm{ED}$ is feasible, faster, and does not require EEG technicians. Thus, it is a desirable alternative to conventional EEG and may improve patients' care in the ED.

\section{K.G. McKenzie Prize for Clinical Neuroscience Research}

\section{Canadian Neurosurgical Society \\ B.02}

Phase I trial of deep brain stimulation of the subcallosal cingulum for treatment-refractory anorexia nervosa

$N$ Lipsman (Toronto)* B Woodside (Toronto) P Giacobbe (Toronto) C Hamani (Toronto) AM Lozano (Toronto)

Background: Anorexia Nervosa (AN) is among the most common psychiatric conditions in young adults and is associated with the highest mortality rate of any psychiatric illness. Current treatment approaches have been largely ineffective in altering the natural history of the most chronic and treatment-refractory patients. 
Deep Brain Stimulation (DBS) has shown promise in modulating activity in neural circuits underlying disordered mood and anxiety, both of which feature prominently in chronic AN. Here we evaluate the use of DBS in AN. Methods: We designed and undertook a Phase I pilot trial of DBS of the subcallosal cingulum in patients with chronic, severe, and treatment-refractory AN. Patients were identified through Canada's largest eating disorders program and underwent baseline medical, psychometric and neuroimaging investigations. Results: At 9-months following surgery, 3/6 patients $(50 \%)$ were at a body-mass-index significantly higher than their historic baseline. Chronic stimulation was associated in responders with significant improvements in mood, anxiety, AN-related rituals and preoccupations and cerebral metabolic changes, consistent with a reversal of baseline abnormalities in AN-relevant structures. Conclusions: DBS appears to be a safe and promising option in patients with chronic and treatment-refractory AN. Additional, larger trials are required to verify the results of this small, open-label study.

\section{K.G. McKenzie Prize for Clinical Neuroscience Research}

\section{Canadian Neurosurgical Society B.04}

\section{Visualizing plasticity in the injured human spinal cord with fMRI}

DW Cadotte (Toronto)* P Stroman (Kingston) D Mikulis (Toronto) $R$ Bosma (Kingston) M Fehlings (Toronto)

Background: Evidence of CNS plasticity after traumatic spinal cord injury has been observed in animal models and human brain fMRI studies. Herein, we provide novel evidence for plasticity of the human spinal cord and demonstrate that these changes persist in persons who fully recover. Methods: Heat $\left(44^{\circ} \mathrm{C}\right)$ was applied to 2 dermatomes above and 2 below the level of SCI. Spinal fMRI data was collected on a $3 \mathrm{~T}$ system $(\mathrm{SSFSE}, \mathrm{TE}=30 \mathrm{msec}, \mathrm{TR}=1 \mathrm{sec})$. Data were analyzed using the general linear model $(\mathrm{P}=0.001)$. We conducted a functional connectivity analysis between the spinal segment stimulated and other regions of the spinal cord and brainstem. Clinical measures included AISA examination. Results: 35 people were examined: 20 control, 9 incomplete SCI and 6 ASIA E patients. We demonstrate that dermatomes of abnormal sensation negatively correlate with the number of active voxels $(\mathrm{R} 2=0.93, \mathrm{p}$ $<0.001)$. The number of intra-spinal connections is significantly higher in incomplete SCI patients in a dermatome of normal sensation, $\mathrm{p}=0.045$. This was also observed in ASIA E patients $(\mathrm{p}=0.03)$. Conclusions: For the first time, we show evidence for spinal plasticity in incomplete SCI patients that persist in those who fully recover from their injury.

\section{The President's Prize}

\section{Canadian Association of Child NeUROLOGY \\ G.06}

\section{Subgroup-specific patterns of recurrence in medulloblastoma}

$V$ Ramaswamy (Toronto)* M Remke (Toronto) E Bouffet (Toronto) $U$ Schüeller (Munich) S Gururangan (Durham) S Dunn (Vancouver) $N$ Jabado (Montreal) C Hawkins (Toronto) RJ Packer (Washington) S Pfister (Heidelberg) A Korshunov (Heidelberg) MD Taylor (Toronto)

Background: Recurrent medulloblastoma remains an enormous treatment challenge is almost always uniformly fatal. Recent studies confirmed that medulloblastoma comprises four distinct subgroups. We sought to delineate subgroup-specific differences in recurrent medulloblastoma. Methods: We identified a screening cohort of all recurrent medulloblastomas at the Hospital for Sick Children between 1994-2012, and subgrouped cases using a nanoString-based gene expression class predication algorithm. Our findings were confirmed through analysis of two independent non-overlapping cohorts. Results: A screening cohort of 30 recurrent medulloblastoma was assembled; 9 with local tumor bed recurrences, and 21 metastatic recurrences. Notably, SHH tumors recurred more frequently in the tumor bed $(8 / 11,73 \%)$ whereas Group 3 and Group 4 recur more frequently with metastases $(16 / 18,89 \%$; p<0.001). Latency to death post recurrence was significantly longer in Group 4 patients $(p=0.03)$. The subgroup-specific location of recurrence was confirmed in a multicenter validation cohort $(\mathrm{p}=0.02, \mathrm{n}=40)$, and an independent validation cohort comprising 96 recurrences (SHH in 21/24 local recurrences, Group 3/4 tumors represented $69 / 72$ metastatic relapses) $(p<0.001)$. Strikingly, in all 40 cases where matched primary and recurrent pairs were available, the subgroup affiliation remained stable at recurrence. Conclusions: Significant differences in the pattern of recurrence exist across medulloblastoma subgroups further highlighting the clinical differences between the four principle subgroups. Intensified local therapy should be considered upon initial treatment for $\mathrm{SHH}$ patients. 


\section{Platform Presentations}

\section{CNS / CSCN Chair's Select Abstract Presentations}

\section{A.01}

Lysine restricted diet as novel therapy for pyridoxine dependent epilepsy

$C$ van Karnebeek (Vancouver)* H Hartmann (Hannover) $S$ Jaggumantri (Vancouver) L Bok (Veldhoven) G Sinclair (Vancouver) $J$ van Hove (Aurora) S Gospe (Seattle) B Plecko (Zurich) S Sylvia (Vancouver)

Background: Pyridoxine Dependent Epilepsy (PDE) classically presents as a neonatal onset epileptic encephalopathy. This cerebral aciduria is caused by deficiency of $\alpha$-aminoadipic semialdehyde dehydrogenase (ALDH7A8), an enzyme facilitating lysine catabolism. Despite adequate seizure control with pyridoxine, neurodevelopmental outcome is poor; $75 \%$ suffer developmental deficits. Our Objective was to evaluate effects of dietary lysine restriction as add-on therapy in PDE patients. Methods: A pilot study with observational n-of-1 cohort design was performed; seven patients were started at mean age six years on lysine restriction 50$60 \mathrm{mg} / \mathrm{kg} /$ day and regular monitoring and outcome assessments during a 12-24mos period. Results: The diet was well tolerated; good compliance, no reports of adverse-events. Reduction of plasma pipecolic acid on diet was $20-67 \%$ of pre-treatment level; for urine $\alpha$-AASA $13-72 \%$, for CSF pipecolic acid $87.2 \%$ and AASA $81.7 \%$. Improvement in age-appropriate skills was observed in 4 out of 5 patients showing pre-diet delays, and seizure control was maintained or improved in 6 out 7 children. Conclusions: This study provides Level 4 evidence that lysine restriction is well-tolerated with significant decrease of potentially neurotoxic biomarkers and potential to improve developmental outcomes. An international PDE consortium has been established to conduct future studies using novel trial methodologies and digital tools (www.pdeonline.org).

\section{A.02}

Magnetic resonance guided focused ultrasound (MRgFUS) for thalamotomy in treatment-refractory essential tremor

N Lipsman (Toronto)* ML Schwartz (Toronto) Y Huang (Toronto) K Hynynen (Toronto) T Sankar (Edmonton) AM Lozano (Toronto)

Background: Essential Tremor (ET) is the most common movement disorder, and is associated with significant functional impairment and disability. Although pharmacologic treatments are available, a substantial proportion of patients remain medicationresistant and thus eligible for surgical neuromodulation. Current options include stereotactic thalamotomy, deep brain stimulation (DBS), and gamma knife radiosurgery (GKRS). Here, we explore a fourth option; magnetic resonance guided focused ultrasound (MRgFUS), a non-invasive, image-guided approach that provides real-time feedback and immediate results. Methods: A pilot trial of MRgFUS thalamotomy for medication-refractory ET was designed.
Eligible patients had their diagnosis confirmed by a movement disorder neurologist and underwent baseline tremor assessment and neuroimaging. Assessments were then repeated at 1- and 3-months following treatment. Results: All enrolled patients experienced immediate tremor improvement following treatment $(\mathrm{N}=5)$. Mean tremor reduction in the dominant (treated) arm was $89 \%$ at 1-month $(\mathrm{N}=4)$ and $78 \%$ at 3 -months $(\mathrm{N}=3)$, with significant associated improvements in functional impairment. There were no serious neurological adverse events, with one patient experiencing persistent paresthesias at 3-months. Conclusions: MRgFUS appears to be a safe and effective means of non-invasively generating thalamic lesions in patients with medication-refractory ET. Procedures were well tolerated, with lesion size and temperature monitored in real time under image-guidance.

\section{A.03}

Titin founder mutation is a common cause of myofibrillar myopathy with early respiratory failure

G Pfeffer (Vancouver)* R Barresi (Newcastle) IJ Wilson (Newcastle) SA Hardy (Newcastle) H Griffin (Newcastle) J Hudson (Newcastle) HR Elliott (Newcastle) AV Ramesh (Newcastle) A Radunovic (London) J Winer (Birmingham) S Vaidya (London) A Raman (Hull) $M$ Busby (Leeds) ME Farrugia (Glasgow) A Ming (Hull) C Everett (London) HC Emsley (Manchester) R Horvath (Newcastle) V Straub (Newcastle) H Lochmuller (Newcastle) PF Chinnery (Newcastle) A Sarkozy (Newcastle)

Objective: Titin gene (TTN) mutations have been described in 8 families with hereditary myopathy with early respiratory failure (HMERF). Some of the original patients had features resembling myofibrillar myopathy (MFM), arguing that TTN mutations could be a cause of MFM. Methods: We studied 127 undiagnosed patients with clinical presentation compatible with MFM. Sanger sequencing for the two previously described TTN mutations in HMERF (p.C30071R in the 119th fibronectin-3 (FN3) domain, and p.R32450W in the kinase domain) was performed in all patients. Results: We identified 5 new families with the p.C30071R mutation who were clinically similar to previously reported cases, and muscle pathology demonstrated diagnostic features of MFM. Two further families had novel variants in the 119th FN3 domain (p.P30091L and p.N30145K). No patients had mutations at p.32450. Conclusions: Mutations in TTN are a cause of MFM, and titinopathy is more common than previously thought. The finding of the p.C30071R mutation in $3.9 \%$ of our study population is likely due to a British founder effect. The occurrence of novel FN3 domain variants, although still of uncertain pathogenicity, suggests that other mutations in this domain may cause MFM, and that the disease is likely globally distributed. We suggest that HMERF due to mutations in the TTN gene be nosologically classified as MFMtitinopathy. 


\section{A.04}

\section{Synchrotron imaging of photothrombotic stroke model in mice}

\section{Z Ivanishvili (Saskatoon)*}

Background: Synchrotron imaging is a new method for studying stroke, which utilizes wide range of monochromatic beams allowing imaging at various levels of tissue organization including inorganic elements and organic molecules. Photothrombotic stroke model is a well-characterized animal model, which has not been imaged using synchrotron. This model provides well-localized reproducible stroke lesions, allowing for accurate targeting of the synchrotron beams. Methods: We imaged photothrombotic stroke lesions in mice with synchrotron using mid-Infrared (mid-IR) and X-ray Fluorescence (XRF) beam-lines. The stroke lesions were imaged at various time points post-induction: 1 hour, 4.5 hours, 6 hours, 1 week, 2 weeks, and 3 weeks. The lesions were characterized using basic immunohistochemistry methods as well, and the results were correlated with the imaging findings. Results: The mid-IR imaging maps showed gradient of oxidized protein, oxidized lipids and glycogen demonstrating "core" of the stroke lesion with surrounding "penumbra" and normal tissue. The XRF maps showed variability in the elemental (Potassium, Calcium, Zinc and Iron) distribution in the "core" across the time points. Conclusions: We present first synchrotron imaging results in photothrombotic stroke model, allowing to map the stroke lesion at the molecular and atomic levels.

\section{CNSS ChaIr's Select Abstract Presentations}

\section{B.01}

\section{Diffusion weighted imaging in the prognostication of glioblastoma multiforme}

A Bata (Halifax) K Ritchie (Haliax) S Walling (Halifax) JJ Shankar (Halifax)*

Background: Glioblastoma Multiforme(GBM) are known to have poor prognosis with no available imaging marker that can predict the survival at the time of diagnosis. Diffusion weighted images (DWI) are used in characterisation of cellularity and necrosis of GBM. The purpose of this study was to assess whether pattern or extent of DWI help in prognositcation of patients with GBM. Material and Methods: We retrospectively analyzed 102 consecutive patients with confirmed GBM on biopsy or resection. Spearman's correlations explored relationships among: total volume of tumor on FLAIR images, post gadolinium T1W images, total volume of tumor showing restricted diffusion on DWI, lowest Apparent Diffusion Coefficient (ADC) in tumor region and in normal-appearing contralateral white matter, age, and survival (days). Cox regression tested for the model that best predicts survival. Results: Two strongest correlations with survival were between age $(-0.335$, p < $0.01)$ and minimum relative $\operatorname{ADC}(0.390, \mathrm{p}<0.001)$. Both age $(\operatorname{Exp}(\mathrm{B})=1.043(95 \% \mathrm{CI} 1.022,1.066)$ and minimum relative ADC value $(\operatorname{Exp}(B)=0.481(0.269,0.86)$ were significant predictors of survival $(\mathrm{p}<0.05)$. Conclusion: We may potentially estimate survival in patients with GBM at the time of diagnosis based on the diffusion characteristics of the tumour irrespective of the treatment received.
B.02

Phase I trial of deep brain stimulation of the subcallosal cingulum for treatment-refractory anorexia nervosa

$N$ Lipsman (Toronto)* B Woodside (Toronto) P Giacobbe (Toronto) C Hamani (Toronto) AM Lozano (Toronto)

Background: Anorexia Nervosa (AN) is among the most common psychiatric conditions in young adults and is associated with the highest mortality rate of any psychiatric illness. Current treatment approaches have been largely ineffective in altering the natural history of the most chronic and treatment-refractory patients. Deep Brain Stimulation (DBS) has shown promise in modulating activity in neural circuits underlying disordered mood and anxiety, both of which feature prominently in chronic AN. Here we evaluate the use of DBS in AN. Methods: We designed and undertook a Phase I pilot trial of DBS of the subcallosal cingulum in patients with chronic, severe, and treatment-refractory AN. Patients were identified through Canada's largest eating disorders program and underwent baseline medical, psychometric and neuroimaging investigations. Results: At 9-months following surgery, 3/6 patients $(50 \%)$ were at a body-mass-index significantly higher than their historic baseline. Chronic stimulation was associated in responders with significant improvements in mood, anxiety, AN-related rituals and preoccupations and cerebral metabolic changes, consistent with a reversal of baseline abnormalities in AN-relevant structures. Conclusions: DBS appears to be a safe and promising option in patients with chronic and treatment-refractory AN. Additional, larger trials are required to verify the results of this small, open-label study.

\section{B.03}

Independent risk factors and risk factor selection modeling for the recurrence of chronic subdural hematomas

A Jack (Edmonton)* CJ O'Kelly (Edmonton) J Findlay (Edmonton)

Introduction: Chronic subdural haematoma $(\mathrm{CSDH})$ recurrence remains a challenge with reported rates approaching $20 \%$. This study investigates independent factors predicting recurrence in a large cohort of $\mathrm{CSDH}$ patients. Methods: We retrospectively reviewed 331 consecutive operative CSDH cases between 20052009. Univariate, multivariate, and statistical selection modeling was completed identifying factors influencing recurrence requiring repeat drainage within 2-months. A model was developed to identify and quantify patients' recurrence risk. Results: The majority of patients were male $(80.66 \%)$. The mean post-operative haematoma volume was $63.55 \mathrm{cc}$. Factors associated with higher post-operative haematoma volume were loculated (septated) CSDHs, pre-operative volume, midline shift, age, and atrophy. All factors except midline shift were independently predictive of larger post-operative haematoma. A $12 \%$ recurrence rate of repeat drainage was found. Factors associated with reoperation were the type of operation, midline shift, pre-operative and post-operative haematoma volume. Loculated CSDHs was the only independent predictor. A scoring system for quantifying recurrence was created based on patient age, haematoma volume, and presence of loculations. Higher recurrence rates were found amongst higher patient scores. Conclusion: Haematoma volume, age, and presence of loculations are found to most strongly influence operative recurrence. A scoring model for identifying higher risk patients for recurrence was developed. 


\section{B.04}

Visualizing plasticity in the injured human spinal cord with fMRI

DW Cadotte (Toronto)* P Stroman (Kingston) D Mikulis (Toronto) $R$ Bosma (Kingston) M Fehlings (Toronto)

Background: Evidence of CNS plasticity after traumatic spinal cord injury has been observed in animal models and human brain fMRI studies. Herein, we provide novel evidence for plasticity of the human spinal cord and demonstrate that these changes persist in persons who fully recover. Methods: Heat $\left(44^{\circ} \mathrm{C}\right)$ was applied to 2 dermatomes above and 2 below the level of SCI. Spinal fMRI data was collected on a $3 \mathrm{~T}$ system $(\mathrm{SSFSE}, \mathrm{TE}=30 \mathrm{msec}, \mathrm{TR}=1 \mathrm{sec})$. Data were analyzed using the general linear model $(\mathrm{P}=0.001)$. We conducted a functional connectivity analysis between the spinal segment stimulated and other regions of the spinal cord and brainstem. Clinical measures included AISA examination. Results: 35 people were examined: 20 control, 9 incomplete SCI and 6 ASIA E patients. We demonstrate that dermatomes of abnormal sensation negatively correlate with the number of active voxels $(\mathrm{R} 2=0.93, \mathrm{p}$ $<0.001)$. The number of intra-spinal connections is significantly higher in incomplete SCI patients in a dermatome of normal sensation, $\mathrm{p}=0.045$. This was also observed in ASIA E patients $(\mathrm{p}=0.03)$. Conclusions: For the first time, we show evidence for spinal plasticity in incomplete SCI patients that persist in those who fully recover from their injury.

\section{CACN Chair's Select Abstract Presentations}

\section{C.01}

Predictors of seizure outcomes in children with tuberous sclerosis and intractable epilepsy undergoing resective epilepsy surgery: an individual participant data meta-analysis

A Fallah (Toronto)* GH Guyatt (Hamilton) C Snead (Toronto) S Ebrahim (Hamilton) GM Ibrahim (Toronto) A Mansouri (Toronto) D Reddy (Hamilton) SD Walter (Hamilton) AV Kulkarni (Toronto) M Bhandari (Hamilton) L Banfield (Hamilton) $N$ Bhatnagar (Hamilton) S Liang (Beijing) F Teutonico (Pavia) J Liang (Shenzhen) JT Rutka (Toronto)

Introduction: We performed an individual participant data metaanalysis to identify preoperative factors associated with a good seizure outcome in children with Tuberous Sclerosis Complex undergoing resective epilepsy surgery. Methods: We searched electronic databases (MEDLINE, EMBASE, CINAHL and Web of Science), and archives of major epilepsy and neurosurgery meetings with no language or date restrictions. We included cohort studies of consecutive participants undergoing resective epilepsy surgery. To identify predictors of a good seizure outcome (i.e. Engel Class I or II) we used logistic regression adjusting for length of follow-up for each preoperative variable. Results: Of 9863 citations, 20 articles reporting on 181 participants were eligible. Good seizure outcomes were observed in $126(69 \%)$ participants (Engel Class I: 102(56\%); Engel class II: $24(13 \%))$. In univariable analyses, absence of generalized seizure semiology $(\mathrm{OR}=3.1,95 \% \mathrm{CI}=1.2-8.2, \mathrm{p}=0.022)$, no or mild developmental delay $(\mathrm{OR}=7.3,95 \% \mathrm{CI}=2.1-24.7$, $\mathrm{p}=0.001$ ), unifocal ictal scalp electroencephalographic (EEG) abnormality $(\mathrm{OR}=3.2,95 \% \mathrm{CI}=1.4-7.6, \mathrm{p}=0.008)$ and EEG/Magnetic resonance imaging concordance $(\mathrm{OR}=4.9,95 \% \mathrm{CI}=1.8-13.5$, $\mathrm{p}=0.002$ ) were associated with a good postoperative seizure outcome. Conclusions: Small retrospective cohort studies are inherently prone to bias, some of which are overcome using individual participant data. We identified four preoperative factors predictive of good seizure outcomes following resective epilepsy surgery. Given the low incidence of children with Tuberous Sclerosis Complex undergoing epilepsy surgery, large long-term prospective multicenter observational studies are required to evaluate these predictors.

\section{C.02}

Ipsilateral corticomotor projections in perinatal stroke: effects of rTMS

O Damji (Calgary)* O Kotsovsky (Calgary) $R$ Chen (Toronto) A Kirton (Calgary)

Background: Unilateral perinatal stroke (PS) causes most hemiparetic cerebral palsy. Developmental neuroplasticity models suggest persistence of ipsilateral projections from unlesioned motor cortex (M1) is potentially maladaptive. Transcranial magnetic stimulation (TMS) can assess ipsilateral neurophysiology while repetitive TMS (rTMS) may modulate it for therapeutic effect. Methods: Children with PS (6-18yrs) were recruited to a clinical trial (PLASTIC CHAMPS) of intensive motor learning therapy and randomization to daily inhibitory rTMS (unlesioned M1, 10 days) or sham. TMS neurophysiology and motor function measures (Melbourne, MA; Assisting-Hand, AHA) were completed at baseline and 1 week post. Outcomes included rest motor threshold (RMT), stimulus response curves (SRC), short-latency intracortical inhibition (SICI), and intracortical facilitation (ICF). Mean change and correlations between ispi- and contra- parameters were analyzed. Results: Of 35 children, 17(49\%) had ipsilateral projections. Affected hand MA scores improved in the rTMS group $(\mathrm{p}=0.045)$. AHA scores were unchanged. Contralateral physiology showed increased RMT $(\mathrm{p}<0.05)$ and decreased SRC slope $(p=0.017)$ after rTMS. Similar changes were observed ipsilaterally. rTMS-induced changes in ipsi- and contra-lateral cortical systems were correlated: SICI $(\mathrm{p}=0.04)$, ICF $(\mathrm{p}=0.009)$. Procedures were well tolerated. Conclusions: rTMS is feasible in PS children. Hand function does not decrease and may improve. rTMS-induced shifts in neurophysiology correlate bilaterally. 


\section{C.03}

Mutations in ATP1A3 cause cerebellar ataxia, areflexia, pes cavus, optic atrophy, and sensorineural hearing loss (CAPOS) syndrome

MK Demos (Vancouver)* C van Karnebeek (Vancouver) C Ross (Vancouver) Y Shen (Vancouver) G Horvath (Vancouver) M Suri (Nottingham) JM Friedman (Vancouver) A Fryer (Liverpool) KM Boycott (Ottawa) SJ Jones (Vancouver)

Background: In 1996, CAPOS syndrome was first described in a single family in which previously healthy individuals developed early onset cerebellar ataxia following a febrile illness, along with progressive optic atrophy and sensorineural hearing loss. All affected family members also had areflexia and pes cavus. The pattern of inheritance was presumed autosomal dominant. A second affected family was identified in our clinic so we aimed to find the disease-causing gene using next generation sequencing. Methods: Whole-exome sequencing was performed on DNA from each of the two index cases to identify rare variants supporting autosomal dominant inheritance. Sanger sequencing was performed to confirm our results. Results: A missense mutation in the $\mathrm{Na}+\mathrm{K}+-$ ATPase alpha3 gene (ATP1A3) was identified and confirmed in both probands. The same mutation was also shown to segregate with disease in both families and targeted sequencing of ATP1A3 in a third affected family with CAPOS syndrome identified the same mutation in this gene. Conclusions: Mutations in ATP1A3 have already been demonstrated to cause Rapid-onset DystoniaParkinsonism and more recently Alternating hemiplegia of childhood-2. This work shows that mutations in ATP1A3 are also responsible for CAPOS syndrome which further expands the spectrum of phenotypes associated with mutations in this gene.

\section{C.04}

Race, ethnicity and geographic distribution of pediatric chronic ataxia in Manitoba

M Salman (Winnipeg)* S Masood (Winnipeg) M Azad (Edmonton) B Chodirker (Winnipeg)

Background: Genetic and environmental factors are important determinants of disease distribution. Several disorders associated with ataxia occur more commonly in certain religious or ethnic groups e.g. disequilibrium syndrome in the Hutterites. Our aim was to determine the racial, ethnic/religious and geographic distribution of pediatric patients with chronic ataxia in Manitoba. Methods: We identified 184 patients with chronic ataxia during 1991-2008 from multiple sources. Their diagnosis, race/ ethnicity and place of residence were determined following a chart review. Results: Most patients resided in Manitoba ( $\mathrm{N}=177)$. Thirty five were Aboriginal, 28 Mennonite and 11 Hutterite. The latter two groups were significantly overrepresented in our cohort. Ataxia telangiectasia, mitochondrial disorders, and non-progressive ataxia of unknown etiology associated with pyramidal tracts signs and developmental delay were significantly more common in Mennonite patients. All four patients with neuronal migration disorders associated with chronic ataxia were Aboriginal. Few isolated disorders with chronic ataxia occurred in the 11 Hutterite patients. Conclusion: Three disorders associated with chronic ataxia were more prevalent than expected in Mennonites in Manitoba. Few rare disorders were more prevalent in the Hutterite and Aboriginal population. Further research is needed to determine the risk factors underlying these variations in prevalence within different ethnic groups.

\section{STROKE}

\section{D.01}

Octogenarians should not be excluded from acute stroke endovascular trials as major clinical responses common with reperfusion

M Almekhlafi (Calgary) J Desai (Calgary) S Mishra (Calgary)* V Nambiar (Calgary) $O$ Volny (Calgary) B Menon (Calgary) M Eesa (Calgary) AM Demchuk (Calgary) M Goyal (Calgary) W Morrish (Calgary)

Background: Octogenarians were excluded from many endovascular stroke trials based on expected delays in achieving successful reperfusion due to tortuous vascular anatomy and a perceived poor potential for recovery. We assessed the safety of the stentrievers technology in those patients. Methods: This is a longitudinal cohort of anterior circulation stroke patients treated in our center. Octogenarians were considered for IA stroke therapy in the absence of a pre-existing disability (Barthel index $\geq 90$ ) or terminal illness. Results: Characteristics are shown in Table 1 below. Octogenarians had a non-significant increase in the in-hospital mortality; all occurred in patients who did not reperfuse successfully. In a multivariable logistic regression, age did not impact NIH improvement in 24-hours. There was a non-significant trend toward increased puncture-to-reperfusion times with increasing age ( $p$ 0.8). Conclusion: Octogenarians can be treated in a safely and fast when selected carefully. The impact of age on longterm functional outcome was not assessed. Excluding these patients from randomized trials of acute stroke therapy may need to be revisited. Table 1 (D.01)

\begin{tabular}{|l|c|c|}
\hline & Octogenarians & Non-Octogenarians \\
\hline $\mathbf{N}$ & $\mathbf{2 0}$ & $\mathbf{6 6}$ \\
\hline Median age (iqr) & $83(4)$ & $65(15)$ \\
\hline Median baseline NIHSS & $17(11)$ & $18(9)$ \\
\hline Median baseline ASPECTS & $9(2.5)$ & $8(3)$ \\
\hline Proportion treated with IV tPA & $60 \%$ & $47 \%$ \\
\hline Median door to imaging & $22(13)$ & $18(9)$ \\
\hline Median imaging to puncture & $60(45)$ & $62(14)$ \\
\hline Median puncture to reperfusion & $42(38)$ & $39(13)$ \\
\hline Median onset to reperfusion & $269(79)$ & $233(184)$ \\
\hline Proportion with successful reperfusion (TICI 2b-3) & $75 \%$ & $83.3 \%{ }^{*}$ \\
\hline Median 24-hour NIHSS & $9(14)$ & $7(10) \dagger$ \\
\hline Median 24-hour ASPECTS & $8(4)$ & $6(3) \dagger$ \\
\hline Median NIH drop in 24 hours & $7(6)$ & $10(10)$ \\
\hline Proportion with 50\% or more drop in NIH in 24 hours & $47 \%$ & $58.5 \%$ \\
\hline In-hospital mortality & $15 \%$ & $6 \%$ \\
\hline & ${ }^{*}$ Chi-square $\mathrm{p}>0.05$ & 十U test p-value $>0.05$ \\
\hline
\end{tabular}

\section{D.02}

Not all successful reperfusion patients are equal: the need for a TICI2c score

$M$ Almekhlafi (Calgary) M Eesa (Calgary) B Menon (Calgary) J Desai (Calgary) S Mishra (Calgary)* V Nambiar (Calgary) $O$ Volny (Calgary) AM Demchuk (Calgary) M Goyal (Calgary)

Objective: Many studies used the TICI system for angiographic outcomes. We propose a new scoring system that separates 
successful but incomplete reperfusion into two categories. Methods: This is a cohort of anterior circulation stroke treated using intraarterial stentrievers in our center. Successful reperfusion was defined as $2 b$ : substantial perfusion with distal branch filling of $\geq 50 \%$ of territory visualized, 2c: near complete perfusion except for slow flow in a few distal cortical vessels, or presence of small distal cortical emboli, 3: complete perfusion with normal filling of all distal branches. Results: In 101 patients, 78 achieved successful reperfusion $(77.2 \%)$. The proportion of patients who had significant improvement of their clinical deficits in 24 hours (24-hour NIHSS score drop by $75 \%$ ) was significantly higher with TICI2c reperfusion than those with TICI $2 \mathrm{~b}$ (50\% vs. $24 \%$, Fisher's exact $\mathrm{p}$ 0.04). Conclusions: Categorizing successful but incomplete reperfusion into 2 different classes has merits. We found evidence toward different short-term clinical and imaging outcomes in patients with TICI2b vs. TICI2c scores. The results are presented in the table below. Table 1 (D.02)

\begin{tabular}{|l|c|c|c|}
\hline & TICl 2b & TICI 2c & TICI3 \\
\hline $\mathrm{N}$ & 32 & 26 & 20 \\
\hline Median age & 67.5 & 67 & 68 \\
\hline Median baseline NIHSS & 18 & 17 & 17 \\
\hline Median baseline ASPECTS & 8 & 8 & 8 \\
\hline Proportion treated with IV tPA & $43.8 \%$ & $50 \%$ & $65 \%$ \\
\hline Median imaging to reperfusion & 115.5 & 96 & 74 \\
\hline Median puncture to reperfusion & 48 & 35 & 33 \\
\hline Median onset to reperfusion & 265 & 218 & 197 \\
\hline & & & 2 \\
\hline Median 24-hour NIHSS & 11 & 5 & 8 \\
\hline Median 24-hour ASPECTS & 5 & 7 & 11 \\
\hline Median NIH drop in 24 hours & 8 & $50 \%$ & $65 \%$ \\
\hline $\begin{array}{l}\text { Proportion with 75\% or more drop in NIH in } \\
\text { 24 hours }\end{array}$ & $24 \%$ & $7.7 \%$ & $5 \%$ \\
\hline $\begin{array}{l}\text { Proportion with 24-hour symptomatic hem- } \\
\text { orrhage (SITS-MOST) }\end{array}$ & $12.5 \%$ & & \\
\hline
\end{tabular}

\section{D.03}

Imaging-to-stent deployment time interval is shorter during daytime hours' vs. evening times in endovascular therapy for acute ischemic stroke

$V$ Nambiar (Calgary)* MA Almekhlafi (Calgary) S Mishra (Calgary) J Desai (Calgary) O Volny (Calgary) AM Demchuk (Calgary) M Goyal (Calgary)

Background: Stentrievers can deliver blood to the ischemic brain once the stent is deployed. A potential factor that may introduce delays is the timing of the intervention. We assessed the feasibility of achieving short imaging to stentriever deployment during the daytime hours. Method: This is a cohort of acute endovascular strokes patients. The imaging to first stentriever deployment time was defined as the time from the completion of $\mathrm{CT}$ angiogram to the first angiographic run that shows that the stentriever has been deployed in the target occluded artery. This interval was compared between daytime procedures (0700-1800 hours) vs. outside. Results: 99 patients were analysed. The median imaging to stentriever deployment was 86 minutes (80.5 minutes during the daytime vs. 97 minutes outside; U-test $\mathrm{p}$ value 0.038). The proportion of patients treated during daytime with imaging to stentriever deployment time $<120$ minutes was $86 \%$ vs. $65.8 \%$ outside (Chi-square p 0.02). The proportion of successful reperfusion (TICI $2 \mathrm{~b}$ or 3 ) during daytime was $76.7 \%$ vs.76.9\% outside. None of the patients who did not meet this target time achieved mRS score $\leq 1$ compared to $55 \%$ of those with an imaging to stentriever deployment time $<120$ minutes (Chi-square p 0.002). Conclusions: Imaging to stentriever deployment time $<120$ minutes is feasible and was achieved more consistently during daytime hours. It was associated with better outcomes.

\section{D.04}

Cerebral flow augmentation and outcomes in moderate severity stroke and good baseline ASPECTS

M Eesa (Calgary) A Demchuk (Calgary) J Modi (Calgary) T Stewart (Calgary) B Menon (Calgary) M Almekhlafi (Calgary) A Shuaib (Edmonton) $M$ Goyal (Calgary)*

Background: Small core determined by ASPECTS is an important determinant of outcomes after stroke. Given the variation in stroke-severity; this with ASPECTS can be important factors determining prognosis. Flow augmentation with the NeuroFlo device may be an alternative therapy for patients outside traditional time windows. We present an exploratory post-hoc analysis in patients with small core and moderate-severity stroke as determinants on outcomes from the SENTIS trial. Methods: SENTIS was a randomized, multicenter trial comparing standard therapy with and without NeuroFlo in patients with baseline NIHSS of 5-18 where treatment could be initiated within $14 \mathrm{~h}$. Long-term outcome was measured with 90-day mRS. We compared mRS in patients with NIHSS 8-15 and good baseline ASPECTS(7-10) between treatment and control arms. Results: A total of 515 patients were enrolled. Of these 152 patients had small core and NIHSS 8-15. There were 73 patients in the treated group [mean age 68.0 14.3 ; median NIHSS

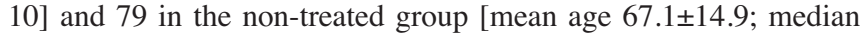
NIHSS 11] and no imbalances in baseline characteristics. mRS 0-2 was seen in $52.2 \%$ of the treatment arm and $41.4 \%$ of the nontreatment arm. A 3-level shift analysis for the three mRS groups 02/3-4/5-6 showed a p-value of 0.14. Conclusions: This post-hoc analysis suggests a trend towards good outcomes in moderate severity stroke with good baseline ASPECTS when treated with NeuroFlo.

\section{D.05}

Achieving an IV needle to arterial puncture time under 60 minutes in acute endovascular stroke therapy is feasible

$S$ Mishra (Calgary)* MA Almekhlafi (Calgary) V Nambiar (Calgary) J Desai (Calgary) O Volny (Calgary) AM Demchuk (Calgary) M Goyal (Calgary)

Background: The IMS-III trial reports an 88-minute time interval from IV tPA administration to arterial groin puncture. We assessed the feasibility of achieving a shorter time interval in routine practice. Methods: This a cohort of acute anterior circulation strokes. Times were prospectively collected. Results: Out of 105 patients, 64 $(60.6 \%)$ received IV tPA. The median age was 64 years with a median NIHSS score of 18 (iqr 9). The onset to IV needle time was 130 minutes (iqr 111). All patients were treated via endovascular therapy with a median and mean times of IV needle to arterial puncture times of 46 and 49.7 minutes, respectively. Of all treated with IV tPA, 39 patients $(60.9 \%)$ had the arterial puncture within 60 minutes of the IV bolus time. Endovascular recanalization was achieved in 78.1\% resulting in a median 24-hour NIHSS score of 6 (iqr 11). Significant improvement in the 24-hour NIHSS scores (drop of $50 \%$ or more from baseline to 24 hours) was noted in 
70.3\%. Conclusion: An IV needle to arterial puncture time under 60 minutes was feasible in about $60 \%$ of patients. Potential delays in randomized trials may include the time needed to obtain consent for enrolment and time to activate the endovascular team. Future trials should still aim to achieve a fast IV needle to puncture time given the potential for clinical improvement.

\section{D.06}

Optimizing acute stroke imaging for maximizing information and minimizing acquisition, post processing and interpretation times: analysis of data from a prospective imaging cohort study $M$ Goyal (Calgary)* BK Menon (Calgary)

Aim: To compare utility and efficiency of perfusion CT (PCT) to NCCT/multiphase CTA (mCTA) paradigm in making treatment decisions in patients with acute ischemic stroke. Methods: mCTA (patent pending) is a new technique and significantly better than conventional CTA. Data is from an ongoing prospective imaging based cohort study of patients with acute ischemic stroke at our center. All patients undergo NCCT followed by mCTA and PCT. Results: In 70 patients (median age 67, 49.3\% male), median baseline NIHSS was 10 (IQR 13) and median onset to CT time in those with witnessed stroke onset was 101 mins (IQR 138). Acquisition and interpretation took $<2$ mins for NCCT, $<5$ mins for mCTA and $<20$ mins for PCT. Uncertainty for IV tPA treatment was present in $10.1 \%$ of patients with NCCT, $1.4 \%$ with mCTA and $15.9 \%$ with PCT. Uncertainty for IA treatment was present in $66.7 \%$ of patients with NCCT but only in $2.9 \%$ with mCTA. Patient motion affected image interpretation in $1.4 \%$ of patients with NCCT and mCTA when compared to $7.2 \%$ with PCT. Agreement between mCTA and PCT for IV tPA was seen in $91.2 \%$ patients $(\mathrm{k}=0.41$, $\mathrm{p}<0.001)$. Conclusion: NCCT with mCTA is a robust tool for making IV and IA treatment decisions in patients with acute ischemic stroke.

\section{D.07}

A systematic comparison of different techniques to measure clot length in patients with acute ischemic stroke

AA Qazi (Calgary)* M Eesa (Calgary) E Qazi (Calgary) M Goyal (Calgary) A Demchuk (Calgary) B Menon (Calgary)

Introduction: Clot length on CT/CTA has been used to predict recanalization with thrombolytic treatment in patients with acute ischemic stroke (AIS). We compared two techniques of measuring clot length on CT/CTA to identify the most reliable method. Methods: 41 patients with M1 MCA occlusions were included from INTERRSeCT (prospective cohort study of AIS patients). Two readers independently measured clot length on CTA by: 1) measuring the occluded segment of M1 MCA and 2) ratio of residual lumen length within M1 MCA to length of contralateral patent M1 MCA. Level of concordance between raters was calculated using Cohen's kappa and Intra-class Correlation Coefficient. Results: Residual lumen ratio on CTA ( $3 \mathrm{~mm}$ ) is the most reliable technique for measuring clot length. Length of hyperdense sign on NCCT is fairly reliable. Direct clot length measurements on CTA are reliable if done on CTA thick slices (24 mm): Conclusion: Reliability of clot length assessment depends on the imaging modality and technique used. CTA still remains the best tool to measure clot length. See Table 1.
Table 1 (D.07)

\begin{tabular}{|l|l|l|c|c|}
\hline \multicolumn{1}{|c|}{ Modality } & Clot Assessment Methodology & & Level of Concordance & p value \\
\hline Non contrast CT & Presence of Hyperdense Sign & & $\mathrm{k}=0.80$ & $<0.001$ \\
\hline Non contrast CT & Presence of Hyperdense Sign & Proximal 1/3rd of M1 MCA & $\mathrm{k}=0.60$ & $<0.001$ \\
\hline Non contrast CT & Presence of Hyperdense Sign & Middle 1/3rd of M1 MCA & $\mathrm{k}=0.66$ & $<0.001$ \\
\hline Non contrast CT & Presence of Hyperdense Sign & Distal 1/3rd of M1 MCA & $\mathrm{k}=0.85$ & $<0.001$ \\
\hline Non contrast CT & Length of Hyperdense Sign & & $\mathrm{ICC}=0.68$ & 0.48 \\
\hline Non contrast CT & $\begin{array}{l}\text { Length of Hyperdense Sign (after } \\
\text { seeing CTA) }\end{array}$ & & $\mathrm{ICC}=0.47$ & 0.001 \\
\hline & & & & \\
\hline CTA 3 mm & Clot Length & & $\mathrm{ICC}=0.27$ & 0.11 \\
\hline CTA 3 mm & Residual lumen ratio & & $\mathrm{ICC}=0.92$ & 0.32 \\
\hline CTA 3 mm & Length of patent lumen & & $\mathrm{ICC}=0.92$ & 0.32 \\
\hline CTA 3mm & Contrast permeation & & $\mathrm{k}=0.2$ & 0.09 \\
\hline & & & & \\
\hline CTA 24mm & Clot length & & $\mathrm{ICC}=0.73$ & 0.61 \\
\hline CTA 24mm & Residual lumen ratio & & $\mathrm{ICC}=0.88$ & 0.01 \\
\hline CTA 24mm & Length of patent lumen & & $\mathrm{ICC}=0.89$ & 0.34 \\
\hline
\end{tabular}

\section{D.08}

Cerebral flow augmentation and outcomes in patients with good baseline ASPECTS and absence of M1 occlusion

$M$ Eesa (Calgary) A Demchuk (Calgary) J Modi (Calgary) T Stewart (Calgary) B Menon (Calgary) M Almekhlafi (Calgary) A Shuaib (Edmonton) M Goyal (Calgary)*

Background: Baseline ASPECTS is an important determinant of outcomes after acute stroke. Outcomes in proximal occlusions are dependent on recanalization. Flow augmentation with the NeuroFlo device may be an alternative outside traditional time windows. We present a post-hoc analysis in patients with small core and mild-tomoderate stroke in the absence of M1 occlusion. Methods: From patients in SENTIS, we included those with NIHSS 5-15, and no M1 occlusion on imaging. We also analyzed a larger cohort of patients with NIHSS 5-10, with the premise of a low likelihood of an M1 occlusion. The baseline ASPECTS was 7-10. 90-day mRS was compared between groups. Results: Of 515 patients, imaging identified 71 patients with ASPECTS 7-10; NIHSS 5-15 and no M1 occlusion. A second analysis was performed on 172 patients with small core (ASPECTS 7-10) and NIHSS 5-10, with a low likelihood of having M1 occlusion. In patients with moderate stroke and no M1 occlusion, $56.7 \%$ in the treatment arm had good outcome vs $42.5 \%$ (control). In the larger group with no vascular imaging $71.6 \%$ in the treatment arm had good outcome (mRS 0-2) compared to $60.9 \%$ (control). 3-level shift analysis showed p-value of $0.09 / 0.24$ respectively. Conclusions: This analysis suggests a trend towards good outcomes in mild-to-moderate stroke with good ASPECTS and a low likelihood of having an M1 occlusion when treated with NeuroFlo.

\section{D.09}

The impact of referral $\mathrm{ABCD} 2$ score on access to resources in a provincial stroke prevention clinic

CL Voll (Saskatoon) CB Gervais (Saskatoon)* B Kwiatkowski (Saskatoon) M Rajput (Saskatoon)

Background: ABCD2 score is used to predict short-term stroke risk among patients with Transient Ischemic Attack (TIA). Stroke Prevention Clinics (SPC) across North America use the ABCD2 score to efficiently triage referred patients. We assessed concordance of $\mathrm{ABCD} 2$ score generated by the referring agent to $\mathrm{ABCD} 2$ score generated by the stroke neurologist at the time of visit to SPC. Methods: 871 patients were seen in SPC between January 2010 to January 2012. 377 patients met the inclusion criteria of receiving 
both a referral $\mathrm{ABCD} 2$ score, and an $\mathrm{ABCD} 2$ score generated by the stroke neurologist. Impact was assessed by comparing actual waiting time to the average waiting time for their corrected score. Results: ABCD2 score concordance existed in $63.4 \%$ of cases (Weighted Kappa $.436,95 \%$ confidence interval .359 - .514). 138 patients were mis-scored by at least one full ABCD2 risk category. 26 patients (6.8\%) were underscored \& 80 patients $(21.2 \%)$ were overscored. On average, underscored patients were seen ten days later, while overscored patients were seen 34 days sooner. Conclusion: Inaccurate referral $\mathrm{ABCD} 2$ scores can lead to higher-risk patients being seen less urgently, and lower-risk patients being seen ahead of those with greater need. Inclusion of precise directions on referral forms, and increased education for referral agents are two methods of improving the accuracy of triage. Table 1 (D.09)

\begin{tabular}{|l|c|c|c|c|c|}
\hline Table 1 & & \multicolumn{3}{|c|}{ ABCD2@ SPC } & \\
\hline & & Semi-Urgent (0-3) & Urgent (4-5) & Emergent (6-7) & Totals \\
\hline ABCD2 Referral & Semi-Urgent (0-3) & $115(59 \%)$ & $20(13.9 \%)$ & $6(15.8 \%)$ & 141 \\
\hline & Urgent (4-5) & $72(36.99 \%)$ & $102(70.8 \%)$ & $10(26.396)$ & 184 \\
\hline & Emergent (6-7) & $8(4.196)$ & $22(15.3 \%)$ & $22(57.976)$ & 52 \\
\hline Totals & & 195 & 44 & 38 & \\
\hline
\end{tabular}

\section{D.10}

Alterations in cerebrovascular reactivity to carbon dioxide in the early phase of subarachnoid hemorrhage do not predict vasospasm in good grade patients

$L$ da Costa (Toronto)* J Fierstra (Zurich) D Houlden (Ottawa) G Rubenfeld (Toronto) M Tymianski (Toronto) JA Fisher (Toronto)

Objective: Subarachnoid hemorrhage (SAH) alters cerebrovascular reactivity (CVR) $\mathrm{CO} 2$, which could be related to increased risk of vasospasm. Bedside methods to monitor CVR are of interest. We report the results of CVR testing in good grade SAH patients using a new method of $\mathrm{CO} 2$ control. Methods: Eighteen SAH patients and 26 controls underwent CVR testing using Transcranial Doppler (TCD) and standardized iso-oxic end-tidal CO2 changes. Tests were performed on average 3.2 days after $\mathrm{SAH}$ when there was no clinical or radiological evidence of vasospasm. Middle cerebral artery blood flow velocity (MCAv), heart rate (HR), blood pressure (BP) and intracranial pressure (ICP) were recorded whenever available. CVR index was calculated as $\triangle \mathrm{MCAv} / \Delta \mathrm{PCO} 2$. The relationship of abnormal CVR and vasospasm was tested. Results: SAH patients had significantly lower CVR ( $p=0.0001)$ PCO2 changes and MCAv was strongly correlated in controls $(r=0.83$, 95\% CI 0.46-1.20) but not in SAH patients $(r=0.071,95 \%$ CI -0.19 $0.33)(\mathrm{p}=0.0027)$. Impaired CVR early after $\mathrm{SAH}$ was not predictive of future vasospasm $(\mathrm{p}=0.2)$. Conclusions: $\mathrm{CVR}$ is impaired in early phases of SAH, when changes in $\mathrm{PCO} 2$ may not correlate with MCAv changes. Despite abnormal CVR in $50 \%$ of the patients, no correlation with later occurrence of vasospasm was found. At least in good grade patients, impaired CVR does not predict future vasospasm.

\section{D.11}

Different strokes for different folks: cerebrovascular diseases among Chinese Canadians-lessons learned over the past 30 years

JY Chu (Toronto)* S Lam (Waterloo)

With the influx of many immigrants to Canada, the population of Chinese Canadians has been steadily increasing over the past 40 years. It is therefore essential to study this population regarding their stroke patterns since in Peoples' Republic of China where most of these Chinese immigrants are coming from, stroke is the leading cause of death.

Over the past three decades, the principal author and his team of researchers has been carrying out systematic, retrospective epidemiological studies in Toronto about the patterns of cerebrovascular diseases amongst Chinese Canadians. The current presentation will be focused on the results of these studies and lessons learned over this period of time. Findings:

1. Chinese Canadians has a higher frequency of hypertension compared to a case-mixed cohort of Caucasians.

2. Chinese Canadians frequently has higher incidence of intracranial small vessel atherosclerotic disease and they tend to have lesser incidence of extracranial vascular stenosis.

3. Chinese Canadians with ischemic strokes tend to have a higher frequency of diabetes mellitus.

4. Chinese Canadians with diabetes mellitus and strokes tends to have a higher hypertension severity scale.

Future prospective population based studies of Chinese Canadians may help to answer a fundamental question about how genes and environmental factors influence the development of strokes amongst this population of immigrants.

\section{SPINE}

\section{E.01}

Spinal instability neoplastic score (SINS) reliability analysis in spine residents and fellows in orthopedics and neurosurgery

M Spiess (Calgary) L Hnenny (Saskatoon) D Fourney (Saskatoon)*

Introduction: The Spinal Instability Neoplastic Score (SINS) was developed to facilitate the diagnosis of neoplastic instability and improve referral patterns to surgery. The purpose of this study was to determine the reliability of SINS in spine trainees. Methods: 25 subjects ( 23 residents [orthopedics $=18$; neurosurgery $=5$ ], 2 fellows) independently scored 30 de-identified cases on two occasions at least 6 weeks apart. Results: (A) Interobserver agreement: The ICC for total SINS score was 0.990, representing near perfect agreement. The Kappa statistics were $0.948,0.739,0.382,0.427,0.550$, and 0.435 for the fields of location, pain, bone quality, alignment, vertebral body collapse, and posterolateral involvement, respectively. The level of agreement was near perfect for location, substantial for pain, moderate for alignment, vertebral body collapse, and posterolateral involvement, and fair for bone quality. (B) Intraobserver agreement: The ICC for total SINS score was 0.907 , representing near perfect agreement. The Kappa statistics were $0.954,0.814,0.576,0.610,0.671$, and 0.561 for the same respective fields. The level of agreement was near perfect for 
location and pain, substantial for alignment and vertebral body collapse, and moderate for bone quality and posterolateral involvement. Conclusion: SINS was highly reliable in spine trainees. Further analysis will be required to determine any differences by level or type of training.

\section{E.02}

Optimizing patient outcomes in the management of spinal cord injuries in Nova Scotia: a retrospective review from 2005-2010

A Dakson (Halifax)* D Brandman (Halifax) G Thibault-Halman (Halifax) S Christie (Halifax)

Background: Studies of acute care aimed at improving outcomes for patients with traumatic spinal cord injuries (SCIs). Nova Scotia presents an ideal setting to study the effect of these interventions, since the population base is relatively constant and all cases are managed at a single tertiary center. Methods: Provincial Trauma Registry was retrospectively reviewed from 2005-2010, investigating the association between management guidelines of SCI and improvements in the American Spinal Injury Association (ASIA) Impairment Scale (AIS) grades. Results: 95 patients were identified. At mean 5-month post-discharge, 20\%, 100\%, $80 \%$ and $5 \%$ of the AIS grades A, B, C, D patients had $\geq 1$ AIS grade improvement. Patients decompressed within 24 hours of injury (OR 2.4, 95\% CI 0.45 - 12.9), having a pre-hospital systolic BP < $90 \mathrm{mmHg}$ (OR 2.1, 95\% $0.03-12.8$ ) and a positive blood-alcohol level (OR $0.3,95 \% 0.02-3.4$ ) was not significantly associated with AIS grade improvement $(\mathrm{P}>0.05)$. Maintaining a MAP $>85 \mathrm{mmHg}$ post-operatively was associated with a significant improvement (OR 7.7, 95\% CI 1.2 - 48.1, p=0.037). Conclusion: This study demonstrates that contemporary clinical improvement may exceed historical literature reports. Specific guidelines surrounding postoperative blood pressure management is associated with improved neurological outcomes.

\section{E.03}

Can a clinical classification of symptoms and signs predict candidacy for lumbar spine surgery?

\section{Wilgenbusch (Saskatoon) D Fourney (Saskatoon)*}

Introduction: The Saskatchewan Spine Pathway utilizes a clinical classification (SSPc) for back and leg pain to triage appropriate referrals for surgery. The objective of this study was to determine how well SSPc predicts an indication for surgery and to compare it to back dominance and leg dominance by VAS score. Methods: A retrospective chart review of 124 consecutive elective referrals for low back and leg pain from a single surgeon's practice was conducted over one year (June 1, 2011-May 30,2012). Results: There was no difference between mean VAS back for back dominant and leg dominant SSPc patterns (6.1/10 vs. 5.6/10 respectively, $\mathrm{p}=0.27$, t-test), and VAS leg did not achieve minimum clinically important differences (5.1/10 vs. 6.4/10 respectively, $\mathrm{p}=0.0147)$. VAS back and leg scores were highly correlated ( $<<0.0001$, Pearson correlation coefficient). Back dominant pain patterns (SSPc 1 and 2) were found in 50 patients $(40.3 \%)$, and only $2(4.2 \%)$ were offered surgery. Leg dominant pain patterns (SSPc 3 and 4) were found in 74 patients $(60.7 \%)$, and $40(54 \%)$ were offered surgery. There was a strong correlation between SSPc and whether or not surgery was indicated $(\mathrm{p}<0.0001$, Chi Square test $)$. Conclusions: This preliminary study suggests that the SSPc is superior to VAS back/leg for triaging referrals to surgery. These finding need to be confirmed in other practice settings to determine generalizability.

\section{E.04}

Can wait times for surgical assessment and imaging be reduced through a spine care pathway?

\section{Wilgenbusch (Saskatoon) D Fourney (Saskatoon)*}

Introduction: In Canada, the wait time for surgical assessment is often longer than the wait for surgery. The Saskatchewan Spine Pathway (SSP) includes triage clinics for imaging and surgical assessment. There is concern that the "extra step" of a triage clinic could lengthen the total wait to see the spine surgeon. Since access to imaging influences the wait to see the spine surgeon, this was also evaluated. Methods: A retrospective chart review of 124 consecutive elective referrals for low back and leg pain from a single surgeon's practice was conducted over one year (June 1, 2011-May 30, 2012). Patient with red flags were excluded. Group A patients were referred from the SSP and Group B were conventional referrals. Results: There were 35 (28.2\%) patients in Group A and 89 (71.8\%) patients in Group B. The mean wait time for surgical assessment was 69.9 days (SD, 46.4, range 37-92) in Group A and 144.0 days (SD 117.3, range 41-219) in Group B ( $\mathrm{p}=0.004)$. The mean wait time for MRI was 29.4 days (SD 22.6, range 14-36) in Group A and 73.8 days (SD 41.4 range, 55-99) in Group B $(\mathrm{p}<0.0001)$. Conclusions: Wait times for MRI and surgical assessment were significantly reduced through implementation of a spine care pathway. Population-based data derived from multiple practice settings is required to confirm these results.

\section{E.05}

A Canadian perspective on neurosurgeon preference for surgical method, graft types, collar usage, physiotherapy and back-towork return times after one-level Anterior Cervical Discectomy (ACD)

$R$ Baweja (Hamilton)* M Bennardo (Hamilton) M Aref (Hamilton) F Farrokhyar (Hamilton) K Reddy (Hamilton)

Background: The purpose of this study is to determine actual neurosurgeon practice in Canada regarding ACD surgical method, collar usage, physiotherapy and back-to-work return times. Methods: A one-page questionnaire of 13 questions was e-mailed to all practicing neurosurgeons in Canada using Survey Monkey. Statistics were calculated using SPSS v. 18 and analysed to determine surgeon preference to surgical method, graft types, hard collar usage and back-to-work return times. Results: A total of 182 surveys were sent with 107 replies resulting in a 59\% response rate. Of responders, 81 preformed ACD procedures and 22 did spine fellowships. ACD procedures correlate with collar usage where fusion surgery alone requires a collar but fusion and plate surgery do not. Similarly, graft types correlate with collar usage where surgeons using autografts recommend collars and those that use allograft or metal/PEEK spacers do not. Surgeons recommend physiotherapy for ACDFP patients except for those with autografts. Back-to-work return time was recommended at 6 weeks for ACDF and ACDFP and for the three main graft types (allograft, autograft and metal/PEEK spacer). Conclusion: We report here current Canadian neurosurgeon 
preference for ACD surgical method, graft types, collar usage, physiotherapy and back-to-work-return times.

\section{E.06}

Epidemiology of spinal infections: chart review of osteomyelitis, discitis and epidural abscesses

JP Switzer (Saskatoon)*

Background: Spinal infections are one of the most difficult, complex, and multi-disciplinary health conditions faced by health professionals. Although numerous literatures have been published, clear guidelines for management are lacking. The purpose of this paper was to gather demographic information of the patients with spinal infections and to determine clinical characteristics of such patients. Methods: Charts from 96 adult patients with osteomyelitis, discitis or epidural abscesses admitted to the Royal University Hospital, the University of Saskatchewan from 2007-2011 were analyzed retrospectively. Results: Of the patients studied, $62 \%$ were male and $32 \%$ female, $35 \%$ of patients required surgery, $45 \%$ were IV drug users, and $15 \%$ had a poor outcome measure. Five variables were isolated as being statistically significant in relation to poor patient outcomes. These were the presence of a neurological deficit, higher white blood cell count, positive biopsy result, shorter time before disease diagnosis, and longer hospital admission. Conclusions: Spinal infections are serious conditions requiring long hospital admissions, extended treatments with antibiotics, and the involvement of numerous specialists. As such, spinal infections create a great burden to the health care system. The variables associated with poorer outcomes would alert clinicians when treating patients with spinal infection.

\section{E.07}

Minimally invasive treatment of spinal cord cysts using a tubular retractor system: case series

\section{BA Yarascavitch (Hamilton)* K Reddy (Hamilton)}

Objectives: Traditional open shunting of syringomyelia and cysts of the spine and spinal canal can be problematic. Minimally invasive treatment is a desirable alternative. We present a series of cases successfully treated with cyst-fenestration, cyst-resection or shunting of spinal cysts by means of a tubular retraction system. Methods: The METRx tubular retraction system (Medtronic) was utilised to provide access to perform a full or hemi-laminectomy and provide access to the spinal canal. Intraoperative microscoy was used to visualize the field. Resection or entry of the cyst was then carried out based on configuration and, if required, a shunt catheter inserted and secured in place. Dural opening and closure were done through the tubular retractor system and closure augmented with fibrin sealant. Results: Two patients underwent syringopleural shunting, two patients had cystoperitoneal shunting of epidural cysts, one patient had cysto-subarachnoid shunting, one patient underwent resection of a cystic lesion, and a seventh patient underwent syrinx fenestration with concurrent terminal ventriculostomy. All patients had radiographic and clinical improvement post-operatively and no complications were encountered. Conclusions: We believe that, although technically challenging, insertion of syringopleural and spinal cystoperitoneal shunts or resection of cystic lesions using minimally invasive techniques can decrease procedure-related morbidity and patient discomfort.

\section{E.08}

Spine surgery referrals redirected through a clinical pathway: effects of non-surgeon triage including imaging utilization

\section{Kindrachuk (Saskatoon) D Fourney (Saskatoon)*}

Introduction: The Saskatchewan Spine Pathway (SSP) includes triage clinics staffed by specialized physiotherapists. There is very limited data regarding the efficacy of non-surgeon triage of lumbar spine surgery referrals. Methods: A retrospective analysis of 87 patients with lower back and leg pain initially referred to a spine surgeon but triaged by the SSP clinic between May 1-November 30, 2011. Diagnosis was by the classification of Hall et al. Pain and disability were scored by visual analog pain scale(VAS), modified Oswestry Disability Index (ODI) and EuroQol EQ5D. Results: 62 $(71.26 \%)$ patients (Group A) were discharged after patient education, self-care advice and/or referral for additional mechanical therapies. 25 (28.74\%) patients (Group B) were referred for surgical assessment. The surgical yield in Group B was $44 \%$, compared to $15 \%$ for all new spine referrals prior to implementation of the SSP: an almost three-fold increase. For the combined cohorts, we estimate that the triage clinic prevented 50/87 (57.5\%) MRI studies. Nonsurgeon triage captured all red flags detected by the surgeon. Patients in Group B were much more likely to have a leg-dominant pain pattern $(\mathrm{p}=0.0088)$ and had significantly greater ODI $(\mathrm{p}=$ $0.0121)$ and EQ5D Mobility $(\mathrm{p}=0.0484)$ scores. Conclusions: This preliminary study suggests that the SSP may reduce unnecessary imaging and surgical referrals.

\section{E.09}

Anterior cervical discectomy with arthroplasty versus arthrodesis for single-level cervical spondylosis: a systematic review and meta-analysis

A Fallah (Toronto)* EA Akl (Buffalo) S Ebrahim (Hamilton) GM Ibrahim (Toronto) A Mansouri (Toronto) CJ Foote (Hamilton) $Y$ Zhang (Hamilton) MG Fehlings (Toronto)

Background: It remains unclear whether anterior cervical discectomy with arthroplasty (ACDA) results in improved patientimportant outcomes compared to anterior cervical discectomy with fusion (ACDF) and whether its widespread use should be advocated. Objective: To perform a systematic review to estimate the effectiveness of ACDA compared to ACDF for patient-important outcomes for single level cervical spondylosis. Results: Of 2804 citations, 9 articles reporting on 9 trials (1446 participants) were eligible. ACDA is associated with a clinically significant lower incidence of neurologic failure $(\mathrm{RR}=0.53,95 \% \mathrm{CI}=0.37-0.75$, $\mathrm{p}=0.0004)$ and improvement in the Neck pain visual analogue scale (VAS) $(\mathrm{MD}=6.56,95 \% \mathrm{CI}=3.22-9.90, \mathrm{p}=0.0001$; Minimal clinically important difference $(\mathrm{MCID})=2.5)$. ACDA is associated with a statistically significant but not clinically significant improvement in Arm pain VAS $(\mathrm{MD}=2.88,95 \% \mathrm{CI}=0.63-5.12, \mathrm{p}=0.01, \mathrm{MCID}=2.5$ ) and SF-36 physical component summary $(\mathrm{MD}=2.28,95 \% \mathrm{CI}=0.40-$ $4.17, \mathrm{p}=0.02, \mathrm{MCID}=4.1)$. ACDA is associated with non-statistically significant higher improvement in the Neck Disability Index Score $(\mathrm{MD}=3.03,95 \% \mathrm{CI}=-0.16-6.21, \mathrm{p}=0.06)$ and lower incidence of ALD requiring surgery $(\mathrm{RR}=0.80,95 \% \mathrm{CI}=0.45-1.41, \mathrm{p}=0.43)$. 
Conclusions: There is no strong evidence to support the routine use of ACDA over ACDF in single-level cervical spondylosis. Current studies lack long-term data required to assess safety as well as surgery for ALD. We suggest that ACDA in patients with single level cervical spondylosis is an option although benefits over ACDF remain in question.

\section{E.10}

Investigating the implementation of acute care guidelines for spinal cord injuries in Nova Scotia from 2005-2010

A Dakson (Halifax) D Brandman (Halifax)* G Thibault-Halman (Halifax) S Christie (Halifax)

Background: In Nova Scotia, all identified SCI patients are referred to a single tertiary center in Halifax. We sought to investigate the implementation of best practice guidelines for the management of traumatic SCI patients. Methods: Nova Scotia Provincial Trauma Registry was retrospectively reviewed from 2005-2010. We investigated the time to surgical decompression, post-operative dietary optimization, chemical prophylactic anticoagulation, cardiovascular support and presence of pressure ulcers. Results: 95 patients with SCI were identified. The mean age was $47.7 \pm 20.5$ years (range: $16-94$ ), with $78 \%$ being male. About $33 \%$ of cases were complete injuries. The mean time to assessment was $9.1 \pm 13.4$ hours. Target mean arterial pressure $>85 \mathrm{mmHg}$ was achieved in $26 \%$ (95\% CI, 15.3-41.1\%) of patients. Operative stabilization was performed on 77 patients, and $42 \%$ occurred within 24 hours of injury. Chemical prophylactic anticoagulation was administered within 48 hours of stabilization in $57.1 \%$ (95\% CI, 47.3-66.5\%). In cases where imaging was indicated to exclude venous thromboembolism, 6.1\% (95\% CI, 0.17-0.20\%) and $24.2 \%$ (95\% CI, 12.8-41.0\%) demonstrated DVT or PE, respectively. $16.3 \%$ (95\% CI, 10.3-24.9) had a documented pressure ulcer. Conclusion: This retrospective review of traumatic SCI from 20052010 provides invaluable information in which to optimize patient care in the acute care setting.

\section{E.11}

A retrospective study of the natural history of central cord syndrome and the role of surgical intervention

D Brandman (Halifax)* A Dakson (Halifax) G Thibault-Halman (Halifax) S Christie (Halifax)

Background: The management of central cord syndrome (CCS) is controversial. There is a perception that CCS patients tend to improve without the need for surgical intervention. The purpose of this study was to compare the clinical improvements of patients with traumatic SCI, both with and without CCS. Methods: Nova Scotia Provincial Trauma Registry was retrospectively reviewed from 2005-2010. Improvement in the American Spinal Injury Association (ASIA) Impairment Scale (AIS) was determined after mean five months follow-up. Results: The study population comprised 96 cases with SCI, subdivided into cases with AIS grade A, non-CCS cases with AIS grades B-D and CCS cases. $88 \%$ of the non-CCS and $65 \%$ of the CCS patients underwent surgical decompression, with mean operative times being $71.6 \pm 137.8$ and $102.9 \pm 144.6$ post injury $(\mathrm{p}=0.45)$. The mean improvements in the mean ASIA motor scores for the three groups were $1.3 \pm 6.5$ and $15.6 \pm 35.0$ and $22.5 \pm 14.6$ $(\mathrm{p}=0.004)$ respectively, with a statistically significant difference only between the CCS and AIS grade A groups ( $\mathrm{p}<0.001) .20 \%, 54 \%$ and $10 \%$ of the patients respectively, underwent an improvement of $\geq$ one AIS grades $(p=0.018, \chi 2=8.0)$. Conclusion: This retrospective review investigates the natural history of CCS and explores the role of surgical intervention on optimizing patient outcome.

\section{Trauma and General Neurosurgery}

\section{F.01}

Diagnostic accuracy of intraocular pressure measurement for detection of raised intracranial pressure: a systematic review and meta-analysis

D Yavin (Calgary)* J Luu (Calgary) DJ Roberts (Calgary) MT James (Calgary) N Jette (Calgary) S Wiebe (Calgary)

Background: Intraocular pressure (IOP) has been proposed as a non-invasive method of intracranial hypertension (IC-HTN) diagnosis. We therefore conducted a systematic review and metaanalysis to determine the correlation between IOP and intra-cranial pressure (ICP) and the diagnostic accuracy of IOP for IC-HTN. Methods: We searched bibliographic databases (MEDLINE, EMBASE, and the Cochrane Central Register of Controlled Trials); references of included studies; and conference abstracts for studies comparing IOP and invasive ICP measurement. Two independent reviewers screened abstracts, reviewed full text articles and extracted data. DerSimonian and Laird methods and bivariate random effects models were used to calculate summary estimates. Findings: Among 355 identified potentially relevant citations, 12 studies enrolling 546 patients were aggregated. The pooled correlation coefficient between IOP and ICP was 0.44 (95\%CI 0.260.63 ; I2 statistic, $97.7 \%, \mathrm{p}<0.001)$. The summary sensitivity and specificity of IOP for IC-HTN were $72 \%$ (95\%CI 36-92) and $87 \%$ (95\%CI 65-96), respectively. The summary positive and negative likelihood ratios were 5.8 (95\%CI 1.3-25.2) and 0.3 (95\% CI 0.1 $1.0)$, respectively. When measures were taken within an hour of another, correlation between IOP and ICP improved. Interpretation: Although a modest aggregate correlation was found between IOP and ICP, the measurement of IOP provides limited diagnostic accuracy for IC-HTN. The significant heterogeneity between studies suggests further investigation is required to determine the clinical utility of IOP in the detection of IC-HTN.

\section{F.02}

Mannitol dosing error during intra-facility transfer for intracranial emergencies

CA Elliott (Edmonton)* M MacKenzie (Edmonton) C O’Kelly (Edmonton)

Background: Mannitol is commonly used to treat elevated intracranial pressure. We analyzed mannitol dosing errors at peripheral hospitals prior to transport to tertiary care facilities for intracranial emergencies. Methods: We conducted a retrospective review of the Shock Trauma Air Rescue Society (STARS) electronic patient database of helicopter medical evacuations in Alberta, Canada between 2004-2012 limited to patients receiving mannitol before transfer. We extracted data on mannitol administration; patient characteristics including diagnosis, mechanism, GCS, 
weight, age and pupils. Results: 120 patients received a mannitol infusion initiated at a peripheral hospital for intracranial emergency (median gcs 6 ; range $3-13$ ). There was a $23 \%$ error rate, including an underdosing rate $(<0.25 \mathrm{~g} / \mathrm{kg})$ of $8.3 \%(10 / 120)$, an overdosing rate $(>1.5 \mathrm{~g} / \mathrm{kg})$ of $7.5 \%(9 / 120)$, and a non-bolus administration rate (>1 hour) of $6.7 \%(8 / 120)$. A process analysis was used to identify potential factors leading to these errors and will be presented. Conclusions: Mannitol administration at peripheral hospitals is prone to dosing error. Our analysis suggests potential strategies, such as a pre transport checklist, to mitigate this risk.

\section{F.03}

Epidural hematoma treated conservatively; when to expect the worse

A Robert (Montreal)* M Basamh (Montreal) M Maleki (Montreal) J Marcoux (Montreal)

Background: The Brain Trauma Foundation's guidelines have defined when epidural hematomas $(\mathrm{EDH})$ could be treated conservatively. But patients need careful monitoring and the literature does not provide clues to identify which are at risk for EDH progression. The goal of our study was to identify those patients. Methodology: A level 1 adult trauma center retrospective review of all EDH patients who were triaged initially for conservative management over five years. Demographic data, injury demographics, coagulation parameters, radiological parameters, conversion to surgery and its timing and outcome were analyzed. Results: 184 patients presented with acute traumatic EDH; 37 required urgent evacuation and two were treated with comfort measures. 125 (or $68.7 \%$ of actively treated) patients were treated conservatively and included in the study. Only $11.2 \%$ of these patients required delayed surgery, with a mean delay of $13 \mathrm{~h}$. Statistical comparison showed that younger age $(p<0.0001)$ and coagulopathy $(\mathrm{p}=0.009)$ were significant risk factors. Gender, ISS, GCS, alcohol level, blood thinner use, location of lesion, associated fractures, midline shift, size of EDH were not. Outcome was similar even if surgery became necessary. Conclusion: The majority of EDH is treated conservatively. Coagulopathy and younger age are significant predictors for conservatively treated EDH patients who need closer observation. However, outcome of urgent evacuation was similar to successful conservative management.

\section{F.04}

Clival fractures in a level one trauma center: a ten year retrospective study

\section{A Winkler-Schwartz (Montreal)* J Marcoux (Montreal)}

Background: Clival fracture (CF) is rare among head traumas. This study examines CF incidence, cause, fracture characteristics and clinical course. Methods: All skull base fracture cases admitted to the Montreal General Hospital between February 2002 and October 2012 were obtained from the Quebec trauma registry. Radiologic data was reviewed for CF. Identified CF was categorized by orientation, location and type. Injury mechanism, clinical presentation and outcome were obtained through retrospective chart review. Results: Of the 1738 patients with skull base fractures, 65 (3.7\%) exhibited $\mathrm{CF}$, representing $1.2 \%$ of traumatic brain injuries admitted. Mean Glasgow Coma Scale (GCS) was 7.8 (3-15). Thirty (46.2\%) demonstrated linear fracture, sixteen $(24.6 \%)$ hairline, ten
(15.4\%) diastatic, and nine (13.8\%) displaced. Diastatic fractures had lower GCS than linear and hairline fractures (4.2 versus 8.1 and 9.8 , respectively; $\mathrm{p}<0.05)$. Thirty-seven $(56.9 \%)$ suffered occipital impact, ten (15.4\%) frontal, five (9.2\%) lateral, three (4.5\%) frontallateral, three (4.5\%) lateral-occipital, and two (3.1\%) multiple locations. Mean Extended Glasgow Outcome Scale was 3.3 (1-7) at discharge. Twenty-four (36.9\%) patients died in hospital. Conclusions: High impact injury, occipital impact site, and linear or hairline fractures often occur with CF. Brain injury and mortality are common. This represents the largest CF population studied.

\section{F.05}

Accurate insertion of external ventricular drains in neuro ICU settings

AA Al Jishi (Montreal)*, D. Sinclair (Montreal), K. Bajunaid (Jeddah

Introduction: An External Ventricular Drain enables us to monitor the ICP and serve as a proxy for assessing cerebral perfusion after an aneurysmal SAH. It has a few pitfalls however such as malpositioning that preclude accurate ICP readings, or injury to vital cerebral structures. We present a novel efficient grading scale to better evaluate adequate EVD positioning after insertion. Methodology \& Results: Data included patients admitted with an aneurysmal SAH July 2006 and May 2009. We included all EVDs that were planned for a frontal horn insertion. An original grading system was developed and applied to each EVD insertion (Grades IIV). Multiple variables are discussed in the platform. Around 160 EVDs were inserted and $60 \%$ of the EVDs were placed in an adequate entry zone. Of these catheters, only $15.66 \%$ landed in the optimum in the vicinity of foramen of Monro (Grade I), $47.59 \%$ were placed elsewhere in the ventricles (Grade II) while $36.75 \%$ were placed extraventricular (Grade III). This latter was associated with a higher risk of traversing vital anatomical structures, EVD malfunction, or more insertion attempts. Conclusion: EVD insertion, based on external surface landmarks is associated with a high chance of missing the intended intraventricular target. We propose this grading system to better aid surgeons in EVD insertion and consider it a useful tool in the ongoing neurosurgical virtual projects .

\section{F.06}

Image guided minimally invasive stereoscopic inter-fascicular evacuation of large spontaneous subcortical intracerebral hemorrhages

\section{H Shane (San Antonio)* J Caron (San Antonio)}

Background: Subcortical intracerebral hemorrhages (SC-ICH) frequently expand along the fibers of the superior longitudinal fasciculus, presenting as ovoid masses with their long axis oriented in the anterior-posterior plane. Lesions $<15 \mathrm{cc}$ have a low mortality, while lesions $>90 \mathrm{cc}$ are often fatal. A subgroup of subjects with $\mathrm{ICH}$ within this range might benefit from minimally invasive SC-ICH evacuation. Method: We describe an innovative minimally invasive surgery (MIS), combining fascicular anatomy, advanced imaging technologies and stereoscopic microsurgery for evacuation of large SC-ICH though a $12 \mathrm{~mm}$ aperture port $\left(\mathrm{VBAS}^{\mathrm{TM}}\right.$, Vycor Medical Inc.). Targeted SC-ICH between $40 \mathrm{ccs}$ and $120 \mathrm{ccs}$ with admission GCS $>5$. Subjects followed with sequential CT and MR scans. 
Results: Of 400 ICHs evaluated (2010-12), 16 SC-ICH met selection criteria. A mean $80 \%$ reduction in hemorrhage size was achieved (mean pre-op volume $82.6 \mathrm{cc} \pm 21.8$, post-op $16.9 \mathrm{cc} \pm 12.9$ ). MR imaging demonstrated minimal injury to long white matter tracts on T2-FLAIR. Effect on cortico-spinal tract anatomy will be demonstrated by DTI. Operative nuances will be discussed to achieve maximal ICH evacuation. Conclusion: Innovative MIS technique for large SC-ICH evacuation is feasible and effective, without significant disruption of subcortical white matter tracts.

\section{F.07}

When is the best time to start blood thinners after traumatic subdural hematoma?

\section{Kia (Montreal)* M Maleki (Montreal) J Marcoux (Montreal)}

Background: Of all the intracranial injuries associated with trauma, subdural hematomas (SDH) have the worse reputation for rebleeding. Therefore, anti-platelet or anticoagulation treatment is withheld for all patients with SDH. However, there are some risks associated with this practice. In the literature, there are no guidelines as to how long it is safe to withhold and when it is safe to restart blood thinners. Methodology: Over 400 patients with traumatic SDH who required blood thinning therapy were reviewed. Demographic and injury-related data, indication for therapy, duration of medication withholding, complications related to delay in initiating therapy, complications related with initiation of therapy were noted. Results: The main indications for anti-platelet therapy were cardiac event and stroke prevention, while the main indications for therapeutic anticoagulation were atrial fibrillation, venous thromboembolic event treatment and cardiac metallic valve. The risk of SDH rebleed is present as long as there is a residual hematoma, especially convexity SDH (8\% requiring surgical intervention). Myocardial infarction and embolic stroke occurred in $1 \%$ of the patients each and within one week of admission. Conclusion: The maximum risk of complication from withholding therapy is during the first week and is low. The risk of SDH progression is higher and more prolonged. Blood thinners should be held in the majority of cases for as long as the $\mathrm{SDH}$ is present.

\section{F.08}

\section{Low amplitude EEG improves flash VEP reproducibility during} surgery

DA Houlden (Ottawa)* CA Turgeon (Ottawa) T Polis (Ottawa) $P$ Bourque (Ottawa) J Sinclair (Ottawa) S Coupland (Ottawa) A Kassam (Ottawa)

Background: Flash visual evoked potentials (FVEPs) are typically not reproducible during surgery. We assessed the relationship between intraoperative FVEP reproducibility and EEG amplitude. Methods: Left then right eyes were stimulated by goggle light emitting diodes, and FVEPs were recorded from $\mathrm{Oz}-\mathrm{Fz}$ ' (International 10-20 system) in 12 patients. Recording bandpass filters were typically $3-100 \mathrm{~Hz}$ but in two patients the FVEP was simultaneously recorded using $3-100 \mathrm{~Hz}, 10-100 \mathrm{~Hz}$ and $30-100$ $\mathrm{Hz}$. The reproducibility of FVEP N70-P100 and the amplitude of the concomitant EEG from $\mathrm{C} 4{ }^{\prime}-\mathrm{Fz}$ were measured. Results: Nine patients had low amplitude EEG $(<30 \mu \mathrm{V})$; reproducible FVEPs (3$100 \mathrm{~Hz}$ bandpass) were obtained from 12 of 13 eyes with normal pre-operative vision. The other three patients had high amplitude
EEG $(>50 \mu \mathrm{V})$; no FVEPs were obtained from 3 of 4 eyes with normal pre-operative vision (the other eye had a non-reproducible FVEP). Raising the low cut filter to $10 \mathrm{~Hz}$ and $30 \mathrm{~Hz}$ progressively reduced EEG and FVEP amplitude but improved FVEP reproducibility. Conclusions: FVEP reproducibility was enhanced in the presence of low amplitude EEG. Raising the low cut filter decreased EEG amplitude and improved FVEP reproducibility.

\section{F.09}

A new vision scale for monitoring neurosurgical inpatients: the McMaster Vision Scale

MH Aref (Hamilton)* A Algird (Hamilton) M Bennardo (Hamilton) A Rodriguez. (Hamilton) F Farrokhyar (Hamilton) L Crevier (Hamilton) K Reddy (Hamilton)

Introduction: This study is being conducted in order to test a new vision scale for bedside monitoring of vision over time in a quantitative method. Current methods for measuring vision are subjective with variation and inconsistencies occurring. Therefore, a simple scale would allow for more consistent and reliable examination. Material and Method: The design consists of a 2-phase cross-sectional study. Using a neuro-ophthalmologist as "gold standard" will allow convergent validity assessment. Each eye is tested separately. Visual acuity, visual field and extraocular movements are scored. Visual acuity is scored from 0 (absent) to 12 (normal 20/20). Visual fields are scored from 0 (absent) to 12 (count fingers in all quadrants). Extraocular movements are scored from 0 (absent) to 6 (full). The maximum score for each eye is 30 . Statistical analysis is completed using SPSS v. 19. Reliability coefficients equivalent to intra-observer, inter-observer, and testretest reliability coefficients are calculated. Results: A total of 11 patients $(\mathrm{N}=22$ eyes) met the inclusion criteria. A Repeated ANOVA model revealed test-retest and inter-observer reliability at $86.8 \%$ and $86.0 \%$, respectively. Overall reliability of the scale test is $92.6 \%$. Conclusions: The McMaster Vision Scale is a valid and reliable method for testing vision of neurosurgical patients. This scale can be administered by a spectrum of health care workers at the bedside with consistent results.

\section{F.10}

Developing an adult hydrocephalus clinic care model: assessing and treating patients with iNPH

MG Hamilton (Calgary)* D Koshly (Calgary) R Prince (Calgary) L Estabrooks (Calgary) G Urbaneja (Calgary)

Introduction: Adult Hydrocephalus patients are managed best by utilizing outcome-based assessment and treatment measures. Our experience developing an Adult Hydrocephalus Clinic in the Canadian Health care system is reviewed. Methods: We report data for patients enrolled in the Calgary Adult Hydrocephalus Clinic (CAHC) from January 2009 to December 2012. A database and registry were used. Results: The number of total patients seen in the CAHC has increased from 100 (26 new) in 2009 to 405 (127 new) per year. These patients represent all four clinical domains of adult hydrocephalus: transition, acquired, "arrested" congenital, and idiopathic normal pressure hydrocephalus (iNPH). Patients with a potential diagnosis of iNPH who were formally assessed increased from $8 / 100$ in 2009 to $37 / 127$ in 2012. Assessment and tretment outcome was primarily assessed using gait and cognitive measures. 
In 2012: 4 / 16 lumbar puncture (LP)-iNPH patient assessments were positive (treated with a VP shunt). 28 potential iNPH patients were evaluated with lumbar drainage (ELD): 22 underwent VP shunt insertion. A positive outcome for treatment with a VP shunt was possible in 24/26 (92\%). Conclusion: The CAHC manages a heterogeneous patient population: iNPH represent $29 \%$ of current new referrals. This experience with an outcome-based assessment and treatment model for patients with potential iNPH illustrates how Adult Hydrocephalus care can be accomplished in the Canadian Health Care system.

\section{F.11}

Defining the epidemiological spectrum of adult hydrocephalus care

$M G$ Hamilton (Calgary)* G Urbaneja (Calgary) D Koshly (Calgary) CM Gallagher (Calgary) $R$ Prince (Calgary) $L$ Estabrooks (Calgary) W Hader (Calgary)

Introduction: There are four common Adult Hydrocephalus subgroups: 1) transition patients with an established pediatric diagnosis and or treatment; 2 ) patients with acquired hydrocephalus; 3) adults with previously untreated/undiagnosed congenital hydrocephalus (APUCH) also known as arrested hydrocephalus); and 4) elderly patients with "normal pressure" hydrocephalus (NPH). Methods: We report data for patients enrolled in the Calgary Adult Hydrocephalus Clinic (CAHC) as of June 2012. A database and registry were used. Information regarding patients treated by none CAHC Neurosurgeons was also assessed. Results: There were 1281 adult hydrocephalus patients identified: 624 patients enrolled in the CAHC, and 657 who had procedures performed by other neurosurgeons: $219(17.1 \%)$ transition patients; 446 (34.8\%) acquired etiology patients; $172(13.4 \%)$ APUCH patients; and 188 (14.7\%) patients with iNPH. $47(27.3 \%)$ of the patients with APUCH have not been treated and $67(38.9 \%)$ have been treated with endoscopic third ventriculostomy (ETV). Neuroendoscopy has been used in 309 adult patients and ETV has been the primary mode of treatment in 224 adult patients (only three patients with NPH). Conclusion: It is important that Adult Hydrocephalus Clinics be inclusive and not limited to patients with a diagnosis of iNPH. Our Adult Hydrocephalus Clinic has identified a heterogeneous patient population. Patients with APUCH are more common then typically appreciated. Neuroendoscopy is an important treatment modality that should be considered for appropriate adults with hydrocephalus.

\section{NeURo-ONCOLOGY AND EDUCATION}

\section{G.01}

Comparative integrated analysis of brain tumor initiating cells and their parent tumors

C Poon (Calgary)* W Wu (Calgary) C Pontifex (Calgary) M AlNajjar (Calgary) HA Luchman (Calgary) C Chesnelong (Calgary) $S$ Weiss (Calgary) J Chan (Calgary) JG Cairncross (Calgary) $M$ Blough (Calgary)

Glioblastoma (GBM) is a uniformly fatal intra-axial neoplasm with a dismal prognosis. It is speculated that brain tumor initiating cell (BTIC) cultures derived from resected GBM better captures its molecular heterogeneity and clinical features than traditional cell lines. As the effect of culture stresses on genomic alterations in BTICs has yet to be elucidated, we sought to inspect the genomic and epigenetic fidelity of BTICs compared to their parent tumors (PTs) with a comparative analysis. Using the Affymetrix 6.0 SNP array and the Illumina HT12 beadarray, we acquired data on copy number alterations (CNAs), loss of heterozygosity $(\mathrm{LOH})$, and gene expression patterns from 12 BTIC lines and their matched PTs. Although BTICs acquired more amplifications and deletions than their PTs, statistical significance was not achieved. However, the magnitude of amplifications with and without $\mathrm{LOH}$ in coding regions of the genome were significantly different. Expression analysis revealed that differentially expressed genes in BTICs were chiefly involved with metabolism, while an integrated analysis demonstrated chromosomal alterations in BTICs were most commonly associated with chromosome 7 amplifications, a location associated with putative oncogenes. BTICs maintain their ancestor's core genome structure, with many changes appearing to be "passenger" alterations. However, statistically significant changes did occur in the form of enhancement of existing genomic modifications. Notably, novel recurring genomic alterations were not observed in BTICs.

\section{G.02}

Regulating tumor metabolism and response to therapy in GBMs with Hexokinase II (HK2)

\section{A Vartanian (Toronto)* G Zadeh (Toronto)*}

We recently demonstrated that Hexokinase II (HK2) is a critical regulator of the Warburg effect in glioblastomas (GBM). HK2depletion in intracranial GBM xenografts resulted in reduction of tumor growth with enhanced overall survival. Thus, we hypothesize that altering tumor metabolism through HK2-depletion can sensitize GBM tumors to microenvironmental stressors such as hypoxia and therapeutics such as radiation (RAD) and/or temozolomide (TMZ). Our results indicated that HK2 loss sensitizes GBM cells to radiochemotherapy under hypoxia, with decreased cell proliferation and increased DNA damage. We utilized an inducible short-hairpinRNA system to direct conditional expression of HK2 at different stages of GBM growth and modulated HK2 concurrent with therapeutics. The conditional loss of HK2 significantly improved the survival of glioma-bearing mice, with further enhancement in RAD and combined RAD/TMZ group. Furthermore, loss of HK2 significantly altered GBM neo-vascularization, with decreased microvascular density. We examined the potential for new vessel formation in the context of low-HK2 and found a significant reduction in the recruitment of bone marrow derived progenitor cells (BMDC) to the vasculature. Our results support HK2's role in GBM metabolism, with further capacity to modulate hypoxia and tumor vasculature in order to adapt to altered tumor microenvironment. Taken together, the synergistic effect upon combining HK2knockdown with RAD/TMZ suggests HK2-depletion as a promising strategy to improve clinical efficacy of current GBM therapies. 


\section{G.03}

\section{The fractal geometry of gliomas}

AD Di Ieva (Toronto)*

Fractal geometry is a valid tool for the analysis of complex systems and natural structures. By means of a fractal-based approach, we analyzed patterns of the microvascular trees of human gliomas, in neuropathology as well as in neuroradiology. We applied computer-aided fractal-based analysis to the morphometric quantification of the geometrical patterns of microvessels shown in: a) CD34 immunotreated slices of II to IV grade human gliomas in vitro; b) SWI patterns of gliomas of patients undergoing to ultrahigh field MR imaging (7 Tesla) in vivo. We found that higher-grade gliomas are more vascularized than lower ones. Furthermore, the existence of different patterns within the same group of tumors (IV grade) was shown, indicating that the glioblastoma is "multiforme" ("multishape"), including its microvascularity. In the neuroradiological step, we analyzed 36 patients by means of SWI 7T MRI, finding higher values of fractal dimension in the higher histological grade groups of tumors, demonstrating the presence of more microvessels and microbleedings in malignant gliomas. Fractal parameters are valid estimators of microvascular geometrical complexity. Gliomas of different grades own specific angiogenic patterns, allowing us to speculate the existence of a specific "microvascular fingerprint" in each class of glioma. We suggest this approach as a tool for quantifying and categorizing different neoplastic microvascular patterns and as a potential morphometric surrogate biomarker for use in clinical practice.

\section{G.04}

Retinal architecture predicts visual outcomes in patients with pituitary macroadenomas

WH Ryu (Calgary)* F Costello (Calgary) J Burton (Calgary) L Rudmik (Calgary) B Mechor (Calgary) $Q$ Al Hinai (Calgary) $Y$ Starreveld (Calgary)

Background: Clinically relevant pituitary lesions affect approximately $1 / 1000$ people. For patients with associated vision loss, the optimal surgical treatment window is not known and there are no validated predictors of visual recovery. The purpose of this study was to determine whether eyes with pre-operative retinal nerve fiber layer (RNFL) loss had worse visual outcomes after surgery than eyes with normal pre-operative retinal architecture. Methods: 20 patients (11 females, mean age 52 years) with visuallysignificant pituitary macroadenomas underwent a neuro-ophthalmic evaluation and spectral-domain optical coherence tomography (OCT) testing pre-operatively and 6-months after surgery. Preoperative RNFL thickness (RNFLT) and macular volumes (MV) were compared between patients with normalized visual function versus persistent visual deficits after surgery. Results: After 6months, only $54 \%$ of patients with normal visual outcomes reported awareness of vision loss at onset, as compared to $100 \%$ of patients with poor visual recovery after surgery. Pre-operative RNFLT (72 vs $84 \mu \mathrm{m} ; \mathrm{p}=0.01)$ and MV (9.2 vs $10.0 \mathrm{~mm} 3 ; \mathrm{p}=0.0007)$ were lower in patients with poor outcomes relative to patients with normal vision after surgery. Conclusions: Awareness of vision loss at presentation and lower pre-operative OCT-measures are associated with worse visual outcomes in patients with surgically treated macroadenomas.

\section{G.05}

Frequent progression of pilocytic astrocytomas in adults: a retrospective review

JD Sharma (Vancouver)* A Nichol (Vancouver) S Yip (Vancouver) B Toyota (Vancouver)

Background: Pilocytic astrocytomas (PAs) are well circumscribed, slow-growing WHO grade I tumors of the CNS. They generally follow a benign course with excellent survival rates. However, recent studies focusing on PAs in adults show high recurrence rates and malignant transformation. We therefore conducted a retrospective review to identify risk factors associated with progression of PAs in adults. Methods: The British Columbia Cancer Registry was searched for all cases of pilocytic astrocytomas treated in adult patients. Tumors that were anaplastic on initial pathology were excluded. Baseline characteristics were recorded. Kaplan-Meier estimates and log-rank tests were used to evaluate the impact of clinical, radiological, and histological characteristics on progression-free survival and overall survival. Results: The search yielded 38 adult patients treated between 1977 and $2009.65 \%$ of patients had progression of their tumors after initial treatment. $32 \%$ of patients died from their disease. Extent of resection and the absence of edema on imaging were significantly associated with an increased progression-free and overall survival. Conclusions: Pilocytic astrocytomas have a high rate of progression in adults. Mortality rates were higher than expected. The presence of edema on initial imaging emerged as a potential negative prognostic factor in PAs.

\section{G.06}

\section{Subgroup-specific patterns of recurrence in medulloblastoma}

$V$ Ramaswamy (Toronto)* M Remke (Toronto) E Bouffet (Toronto) $U$ Schüeller (Munich) S Gururangan (Durham) S Dunn (Vancouver) $N$ Jabado (Montreal) C Hawkins (Toronto) RJ Packer (Washington) $S$ Pfister (Heidelberg) A Korshunov (Heidelberg) MD Taylor (Toronto)

Background: Recurrent medulloblastoma remains an enormous treatment challenge is almost always uniformly fatal. Recent studies confirmed that medulloblastoma comprises four distinct subgroups. We sought to delineate subgroup-specific differences in recurrent medulloblastoma. Methods: We identified a screening cohort of all recurrent medulloblastomas at the Hospital for Sick Children between 1994-2012, and subgrouped cases using a nanoString-based gene expression class predication algorithm. Our findings were confirmed through analysis of two independent non-overlapping cohorts. Results: A screening cohort of 30 recurrent medulloblastoma was assembled; 9 with local tumor bed recurrences, and 21 metastatic recurrences. Notably, SHH tumors recurred more frequently in the tumor bed $(8 / 11,73 \%)$ whereas Group 3 and Group 4 recur more frequently with metastases $(16 / 18,89 \%$; $\mathrm{p}<0.001)$. Latency to death post recurrence was significantly longer in Group 4 patients $(\mathrm{p}=0.03)$. The subgroup-specific location of recurrence was confirmed in a multicenter validation cohort $(\mathrm{p}=0.02, \mathrm{n}=40)$, and an independent validation cohort comprising 96 recurrences (SHH in 21/24 local recurrences, Group 3/4 tumors represented $69 / 72$ metastatic relapses) $(\mathrm{p}<0.001)$. Strikingly, in all 40 cases where matched primary and recurrent pairs were available, the subgroup affiliation remained stable at recurrence. Conclusions: Significant differences in the pattern of recurrence exist across 
medulloblastoma subgroups further highlighting the clinical differences between the four principle subgroups. Intensified local therapy should be considered upon initial treatment for $\mathrm{SHH}$ patients.

\section{G.07}

Establishing a curriculum for the Canadian neurosurgery rookie camp: a Canadian national survey

DM Brandman (Halifax)* F Haji (London) M Matte (Halifax) DB Clarke (Halifax)

Background: The first Canadian 'rookie camp' was developed by residents, program directors and representatives from the Royal College of Physicians specialty committee on Neurosurgery. In the months leading up to the camp, we performed a national needs assessment to develop a focused curriculum. Methods: A survey was conducted of all Canadian academic neurosurgical centers, targeting incoming and current PGY-1 neurosurgical residents as well as Program Directors (PDs). A web-based questionnaire consisting of 61 anchored Likert scale and 8 free-text questions was developed. Questions were based on the CanMEDS competency framework and focused on skills relevant to neurosurgical training at the PGY-1 level. Results: 52 individuals were contacted and 38 responses were received. 10 responses were from PDs (10/14, or $71 \%), 11$ from current PGY-1 residents (11/19, or 58\%) and 17 from incoming PGY-1 residents (17/19, or $89 \%)$. Residents and PDs identified similar educational objectives. Areas of emphasis included common clinical scenarios such as status epilepticus and foundational skills such as performing a neurological examination or interpreting radiographic imaging; in contrast, skills related to advanced operative equipment such as endoscopes were considered less important. Free text answers were concordant with the quantitative data. Conclusion: We surveyed Canadian neurosurgical PDs and PGY-1 residents to identify critical areas to be incorporated as part of the curriculum for a Canadian Neurosurgical 'rookie camp'.

\section{G.08}

Resident migration patterns: did changes in ABNS eligibility affect resident destinations?

\section{$A D T u$ (Vancouver)* B Toyota (Vancouver)}

Background: The American Board of Neurological Surgery (ABNS) is the body awarding certification of competency in neurological surgery in the USA. While it does not define practice privilege, recognition is necessitated by many institutions. Application was open to residents entering Canadian programs prior to 1997; however has since been only available to Accreditaion Council for Graduate Medical Education (ACGME) recognized programs, excluding Canadian schools. This study asks whether this change has affected education and employment opportunities for graduating neurosurgeons. Methods: All neurosurgical residency programs in Canada were invited to participate. Programs were asked to provide lists of graduates from 2012 to 1984. Graduates were sorted by year of graduation into pre or post 2003 (ie. six years after 1997) and further training and employment information, if not provided, was gathered from public domain resources. Results: $50 \%$ of programs participated in this study for 220 graduating residents. There was no difference in proportion of American fellowships obtained relative to graduation date. The proportion of American consultant positions declined from $36.4 \%$ to $28 \%$ after 2003 . This difference was statistically significant. Conclusion: Changes in ABNS eligibility did not affect the proportion of American fellowships while consultancy positions did decrease. The aetiology may be multifactorial, including coinciding evolving economics and hiring trends. Future study may delineate the other factors that influence migration and assist future residency planning.

\section{G.09}

Teaching for the transition: the Canadian neurosurgery 'rookie camp' experience

FA Haji (London)* D Brandman (Halifax) M Matte (Halifax) $S$ deRibaupierre (London) S Brien (Ottawa) DB Clarke (Halifax)

Background: The recent AFMC-FMEC report calls for the development of a national orientation program to standardize entry into residency training. To address this goal, a national, simulationbased 'rookie camp' was designed to foster acquisition of essential technical, cognitive and behavioral skills among incoming Canadian PGY-1 neurosurgery residents. Methods: A needs-based curriculum was developed collaboratively by program directors, residents and Royal College staff. Sessions used high and low-fidelity simulation to explore common clinical presentations (spinal cord injury, raised ICP, etc.) and addressed multiple CanMEDS competencies. Program evaluation included: participant and faculty feedback; pre-, post-, and retention knowledge tests; and follow-up interviews to determine clinical behaviour change among participants. Results: 17 of 23 (74\%) PGY-1 residents participated in the camp. All indicated the course helped prepare them for residency training. Test scores revealed a statistically significant improvement in knowledge related to course content $(\mathrm{p}=0.017)$, but not unrelated content $(\mathrm{p}=$ 0.453 ), with no significant difference in retention-test scores at three months $(p=0.783)$. Post-course interviews are underway. Conclusions: A simulation 'rookie camp' for PGY-1 neurosurgical residents was delivered, engaging participants, training programs, specialty societies and accreditation bodies at a national level. The program has promoted knowledge-retention, skill development and facilitated the transition from clerkship to residency by fostering socialization to the specialty.

\section{G.10}

Neurosurgeons' perspectives on surgery for psychiatric disease and enhancement

D Mendelsohn (Vancouver)* N Lipsman (Toronto) M Bernstein (Toronto)

Background: Deep brain stimulation for psychiatric indications is becoming increasingly safe and effective. Given the troubled history of the field and its current renaissance, it is important to evaluate current practices and to gauge cultural attitudes towards invasive neuromodulation. Methods: We designed an online survey distributed electronically to 1180 functional neurosurgeons globally. We measured general and regional trends in functional neurosurgery, and focus specifically on surgery for mind and mood. Results: One hundred ninety surveys were returned $(16.1 \%)$. Psychiatric indications are the most frequently treated conditions for $28 \%$ of respondents. Obsessive-Compulsive Disorder (OCD), and depression are the most common psychiatric disorders treated. The majority of respondents $(81 \%)$ felt optimistic about the future of 
neurosurgery for psychiatric disease (NPD). The reluctance of psychiatrists to refer patients was viewed as the greatest obstacle facing the field. In response to hypothetical scenarios involving cognitive and personality enhancement, opinions varied but the majority opposed enhancement interventions. Regional variations uncovered distinct attitudinal differences depending on geographic location. Conclusions: Most neurosurgeons see neurosurgery for psychiatric disease continuing to grow and psychiatric surgery becoming a larger part of their daily practice. Optimism towards the future of NPD predominates with a general reluctance towards surgery for enhancement purposes.

\section{G.11}

The level of evidence of the neurosurgical literature published in the highest impact clinical journals: where has all the good evidence gone?

BA Yarascavitch (Hamilton)* K Bhanot (Hamilton) D Jacobson (Hamilton) SA Almenawer (Hamilton) JE Chuback (Hamilton) $K$ Reddy (Hamilton) M Bhandari (Hamilton)

Background: Level of evidence (LOE) is well utilized across surgical specialties. We aimed to categorize the LOE of Neurosurgery related papers over a one year period across three clinically focused journals with very high impact factors; New England Journal of Medicine, Lancet and Journal of the American Medical Association. Methods: Clinical neurosurgery literature published in the highest impact clinical journals (HICL) between April 2011 and March 2012 was reviewed. Demographic data was collected and LOE ratings performed in duplicate using the Oxford LOE scale. This was compared to a reference work of clinical studies from top clinical neurosurgical journals in 2010. Results: 4612 articles were screened and 12 met our eligibility criteria. Half of all of studies were Level I evidence $(n=6)$ and of the remaining studies, one was Level II, two were Level III and three were Level IV publications. We found the mean LOE in the neurosurgical HICL was 2.17 which compared favorably to neurosurgical journals (mean 4.0). Conclusions: Overall, there is a paucity of neurosurgical literature published in the HICL. The small amount of literature this is published in the HICL does have a greater proportion of higher LOE studies.

\section{EPILEPSY}

\section{H.01}

Using StatNet EEGs in the emergency department shortens diagnosis delay

F Moien Afshari (Saskatoon)* JA Edlow (Boston) HT Susan (Boston) M Barnard (Boston) TD Pang (Boston)

Background: Delays in diagnosing non-convulsive status epilepticus increases emergency department (ED) mortality. The conventional EEG set up is time consuming even by well-trained technologists. The StatNet electrode can be applied after minimal training. Methods: Hypothesis is that StatNet EEG decreases EEG recording and interpretation delay and facilitates patients' disposition from the ED. We compared several parameters between StatNet EEG with control and historical control (HC) using one-way ANOVA. Results: EEG set up was shorter in the StatNet $(34 \pm 4$,
$\mathrm{N}=20)$ vs. control $(25 \pm 3.3, \mathrm{~N}=24, \mathrm{p}<0.05)$. The delay from EEG order to completion was the shortest in the StatNet group ( $86 \pm 9.3$, $\mathrm{N}=25)$ vs. control group $(170 \pm 25, \mathrm{~N}=25)$ vs. $\mathrm{HCs}(225 \pm 46, \mathrm{~N}=23$, $\mathrm{p}<0.01)$. Time from EEG completion to interpretation was shorter for StatNet $(39 \pm 7, \mathrm{~N}=25, \mathrm{p}<0.001)$ and control $(59 \pm 5, \mathrm{~N}=21$, $\mathrm{p}<0.01)$ groups vs. HCs $(158 \pm 48, \mathrm{~N}=7, \mathrm{p}<0.01)$. Finally, time from EEG order to ED disposition was shorter in the StatNet $(185 \pm 19$, $\mathrm{N}=20, \mathrm{p}<0.001)$ and control $(180 \pm 19, \mathrm{~N}=15, \mathrm{p}<0.01)$ vs. HCs $(374$ $\pm 37, \mathrm{~N}=19)$. Conclusions: We showed that using StatNet EEG in ED is feasible, faster, and does not require EEG technicians. Thus, it is a desirable alternative to conventional EEG and may improve patients' care in the ED.

\section{H.02}

\section{Insulectomy for refractory epilepsy}

\section{A Bouthillier (Montreal)* DK Nguyen (Montreal)}

Purpose: Wilder Penfield was the pioneer of insulectomies for refractory epilepsy, notably when electrocortigraphy performed after temporal lobe removal disclosed insular spikes. However, this approach was abandoned because of poor results and complications. With technological advancement in seizure focus localisation and microsurgery, it is time now to reassess the surgical outcome of insulectomies. Methods: All patients with intracranial insular recordings and all patients who had an insulectomy for refractory epilepsy at the Université de Montreal were reviewed. Insular surgeries for tumors and vascular lesions were excluded. Results: Thirty patients had intracranial insular recordings. Five had electrode placement by stereotaxy and 25 by direct implantation after microsurgical opening of the Sylvian fissure, including ten with the Hybrid Operculo-Insular Electrodes. No patient had complications with permanent consequences. Twenty-one patients had an insular resection, combined with an opercular resection in 19. Seven insulectomies were performed on the dominant hemisphere. Preoperative insular MRI was normal or non specific in $14 / 21$ patients $(67 \%)$. Engel class I seizure outcome was achieved in $18 / 21$ patients $(86 \%)$ (mean follow-up four years, range 0,1-14). No new permanent deficits were detected. Conclusion: Intracranial EEG sampling of the insula and insulectomy for refractory epilepsy are safe procedures. Good seizure control is now a reachable goal.

\section{H.03}

Outcomes in a Canadian single seizure clinic. A one year prospective study

$K$ Watherhouse (Saskatoon)* L Hernandez-Ronquillo (Saskatoon) G Hunter (Saskatoon) F Moien-Afshari (Saskatoon) JF Tellez-Zenteno (Saskatoon)

Background: The assessment of patients with single unprovoked seizures should be done within two weeks of referral and the diagnosis should be confirmed ideally by a consultant with expertise in epilepsy. Methods: We performed a one year prospective study including all patients who were referred to the single seizure clinic in Saskatoon from November 2011 to December 2012. Three certified epileptologists and a registered nurse were responsible for the diagnosis and treatment of patients. Results: Eighty patients were included in this study. The mean age of patients was $38.2+17.8$ (range 14-84). Forty seven percent were females. The wait time from the spell to the assessment was $26+31$ days (range 2-202). Patients 
were referred mostly for emergency room physicians (52\%), 38\% for family $(38 \%)$ and neurologist (10\%). Seventy five percent of patients had diagnosis at the first visit. The diagnoses were as follow: Epilepsy was diagnosed in $42.5 \%$ of patients. Twenty five percent of patients had syncopes, provoke seizures in $12.5 \%$ (alcohol withdrawal, drugs use and hypoglycemia), single seizures in $11 \%$ and non-epileptic events in 9\%. Medications more commonly prescribed were lamotrigine and topiramate. Conclusions: Our clinic has produced a tremendous benefit for patients in our province providing the best care for patients. In all patients the assessment and conclusion was done in the next month after the spell.

\section{H.04}

MRI changes in refractory status epilepticus: is isoflurane neurotoxic?

KM Ikeda (London)* R Connors (New York) J Claassen (New York) $S$ Mirsattari (London) B Young (London)

Background: Refractory status epilepticus (RSE) is associated with high morbidity and mortality. Experts recommend aggressive therapy with continuous intravenous infusions or inhaled anesthetics such as isoflurane. However, there is concern that MRI changes in RSE reflect isoflurane neurotoxicity. We performed a case-control study to address this issue. Methods: We reviewed 21 age- and sexmatched patients with RSE: 11 cases treated with isoflurane and 11 controls treated with intravenous anticonvulsants alone, who underwent MR imaging. MRI reports were reviewed for abnormalities in the meninges, cortex, white matter, basal ganglia, thalamus, hippocampus, brainstem and cerebellum. Results: Abnormalities were identified in nine $(90.0 \%)$ cases and eight $(72.7 \%)$ controls $(\mathrm{p}=0.59)$. Hippocampal abnormalities were reported in five $(50.0 \%)$ cases and one $(9.1 \%)$ control $(\mathrm{p}=0.06)$. All patients with NORSE had hippocampal abnormalities (three cases). There were no significant differences between groups for any other abnormalities ( $\mathrm{p}=0.31-1.0)$. Conclusions: Our results do not support extra-hippocampal neurotoxic potential of isoflurane when used in RSE. The hippocampal abnormalities seen in our cases may be the consequence of a previously reported association with the NORSE syndrome, but an independent association with isoflurane use cannot be excluded on the basis of these data alone.

\section{H.05}

Improved seizure outcomes in resections involving the orbitofrontal cortex: evidence for "orbito-frontal-plus" epilepsy

$D$ Serletis (Cleveland)* JA Gonzalez-Martinez (Cleveland) J Bulacio (Cleveland) I Najm (Cleveland) W Bingaman (Cleveland)

Introduction: Surgery for frontal lobe epilepsy has poor seizure outcomes, likely due to incomplete resection of the epileptogenic zone (EZ). Specifically in orbito-frontal (OF) epilepsy, we identified two pathological and electro-clinical patterns requiring different surgical approaches. We present here a consecutive series of patients with OF epilepsy, highlighting different surgical strategies and correlation with seizure outcome. Methods: Eleven patients with refractory epilepsy involving the $\mathrm{OF}$ region were prospectively identified over three years, in whom stereoelectroencephalography (SEEG) was required to identify the EZ. All patients underwent preoperative evaluation, SEEG and post-operative MRI. Demographic and clinical features were analyzed, and surgical outcome was correlated with type of resection. Results: Post-operative seizure control improved for all patients, with no complications or mortalities. Five patients exhibited "OF-plus frontal polar epilepsy", with the EZ residing in the frontal lobe; following surgery, four were seizure-free and one improved. The remaining six patients had multi-lobar epilepsy, with the EZ in the OF cortex associated with the temporal pole ("OF-plus temporal polar"). Post-operatively, all six were seizure-free (mean follow-up of 26.6 months). Pathology confirmed focal cortical dysplasia (FCD) I in ten patients, with FCD II identified in the remaining patient. Conclusions: In patients with OF epilepsy, multi-lobar resections may be needed to achieve seizure freedom. Failures in frontal resections can be partially explained by incomplete resection of the EZ by leaving behind the temporal pole.

\section{H.06}

Impact of mild cognitive impairment on health-related quality of life in Parkinson's disease

$W$ Reginold (Toronto)* S Duff-Canning (Toronto) C Meaney (Toronto) MJ Armstrong (Toronto) S Fox (Toronto) B Rothberg (Toronto) C Zadikoff (Chicago) N Kennedy (Chicago) D Gill (Hershey) $P$ Eslinger (Hershey) $F$ Marshall (Rochester) $M$ Mapstone (Rochester) K Chou (Anne Arbor) C Persad (Anne Arbor) I Litvan (Louisville) B Mast (Louisville) D Tang-Wai (Toronto) A Lang (Toronto) C Marras (Toronto)

Background/Aims: Assess the impact of mild cognitive impairment or cognitive decline on health-related quality of life (HR-QOL) in Parkinson's disease. Methods: HR-QOL measured by the Parkinson Disease Quality of Life Questionnaire (PDQ-39), mild cognitive impairment according to Level 2 Movement Disorder Society Task Force criteria and cognitive decline from premorbid baseline (Wechsler Test of Adult Reading) were assessed in nondemented patients with Parkinson's disease at six academic movement disorders clinics. Results: Among 137 patients, after adjusting for education, gender, disease duration, and Movement Disorder Society Unified Parkinson's Disease Rating Scale total score, mild cognitive impairment was associated with worse scores within the PDQ-39 dimension of communication. Scores in the dimension of stigma were worst in the second tertile of cognitive decline from premorbid estimates. Mild cognitive impairment was associated with worse social support scores in the second tertile of cognitive decline but not in other tertiles. Conclusion: Mild cognitive impairment and cognitive decline from estimated baseline are associated with reduced health-related quality of life in one or more of communication, stigma, and social support domains. Degree of cognitive decline from baseline appears to modify the association between mild cognitive impairment and HR-QOL in PD and knowing both will allow a better appreciation of the difficulties patients could be facing in daily life. 


\section{H.07}

\section{Demonstration of corticospinal tract pathology in ALS using high field diffusion tensor tractography}

$K$ Bishay (Edmonton)* P Bishay (Edmonton) P Seres (Edmonton) $S$ Kalra (Edmonton)

Background: Amyotrophic lateral sclerosis (ALS) is a rapidly progressive and fatal neurodegenerative disease with a hallmark of upper motor neuron (UMN) and lower motor neuron (LMN) degeneration. Currently, there is no objective and standardized means of UMN assessment. The primary goal of this study is to explore the ability of diffusion tensor imaging (DTI) performed at high field to detect pathological changes in the corticospinal tract (CST) of ALS patients. Methods: Ten ALS patients and fourteen healthy controls were scanned on a high-field (4.7 tesla) MRI scanner. Blinded post-processing of DTI data yielded fractional anisotropy (FA) maps, which were used as a measure of white matter integrity. Manual deterministic tractography was performed to isolate the fibres contributing to the CST from the precentral and postcentral gyri for region of interest (ROI) analyses using the FA maps. Unilateral and bilateral mean FA was calculated for the CST with and without inclusion of fibres originating from the postcentral gyrus. Results: Non-parametric analysis of all four ROIs (unilateral and bilateral CSTs with and without inclusion of fibres from the postcentral gyrus) showed significantly reduced FA in patients (all $\mathrm{p}<0.002$ ). Conclusions: These results suggest that high-field DTI may be useful in developing an objective UMN assessment for ALS patients as well as elaborating on our understanding of pathological changes associated with the disease process.

\section{H.08}

Distinguishing amyloid positive from amyloid negative mild cognitive impairment patients

\section{H Chertkow (Westmount)*}

Background: Amnestic Mild Cognitive Impairment is a high risk state for progression to dementia. According to new NIH criteria, presence of brain amyloid indicates "MCI of the Alzheimer's Type". We wished to ascertain the cause of MCI in individuals lacking amyloid. Methods: 46 patients all meeting criteria for amnestic MCI were studied and compared with 23 age-matched normals. Cognitive decline was documented with MoCA and neuropsychological evaluation. All were studied with PET amyloid imaging with PIB (Pittsburgh B Compound). PIB results were dichotomized as positive/negative according to SUVR cut-offs of 1.4. Exhaustive chart reviews and imaging were carried out to ascertain possible causes of the MCI. Results: $22 \mathrm{MCI}$ individuals were PIB "positive" and 24 MCI individuals were PIB "negative". All the normal controls were PIB negative. These groups were equivalent on age, education, MMSE and MoCA. PIB negative MCIs showed significantly more Cardiovascular risk factors, sleep apnea, and a greater load of psychiatric illness. They had mean 3.5 "cognition-related" morbidities, vs. 1.9 in PIB positive individuals. PIB positive MCIs had more cognition "unrelated" morbidities, consistent with a "frailty" model for AD. Conclusions: The PIB negative MCI subjects represented a heterogeneous group. Many PIB negative subjects had more than one possible cause of cognitive impairment, often with reversible factors that might be amenable to therapy.
H.09

The case for vitamin D supplementation in Multiple Sclerosis

A Ganesh (Calgary)* S Apel (Calgary)* L Metz (Calgary) S Patten (Calgary)

Introduction: Given vitamin D's immunomodulatory role and association with developing Multiple Sclerosis (MS), it may also influence disease activity in MS. We conducted a systematic review examining: (1) vitamin D's role in MS activity, and (2) its therapeutic supplementation in MS. Methods: A comprehensive search of Medline, Embase, Pubmed, and unpublished abstracts yielded 57 studies for appraisal. The evidence was interpreted in light of the Bradford-Hill causation criteria, and the number needed to treat (NNT) to prevent one patient from relapsing annually was calculated for studies examining relapse rate. Results/Discussion: Cross-sectional and longitudinal studies have demonstrated a strong negative correlation between higher vitamin D levels and risk of relapse and/or disability, and higher inflammatory markers in MS. Thus, vitamin D fulfills Bradford-Hill criteria for strong consistent association, biological plausibility, and coherence. Those of temporality, dose-response, and experimental evidence are yet to be adequately met, although preliminary evidence from randomized clinical trials (RCTs) suggests supplementation can reduce relapse rates and/or disease burden. Published data on relapse prevention from heterogeneous studies indicates the possibility of small NNTs of 1.36-25.00. Conclusions: Ultimately, current evidence does not permit inference of a causal relationship between vitamin D deficiency and MS activity. Supplementation appears to be therapeutically promising, but given the paucity of RCTs with placebo/comparator arms, the evidence is not definitive and dosing remains uncertain.

\section{H.10}

Histopathology of Multiple Sclerosis related trigeminal neuralgia following multiple rhizotomies and Gamma Knife surgery

D Phillips (Winnipeg)* M Del Bigio (Winnipeg) A Kaufmann (Winnipeg)

In this report, we describe the pathological changes in trigeminal nerve rhizotomy excisions from three cases of multiple sclerosisrelated trigeminal neuralgia (MS-TN) after Gamma Knife radiosurgery. Our observations are compared one patient with typical TN who underwent partial surgical rhizotomy following failure of Gasserian injury procedures and normal trigeminal nerve from autopsy specimens. The non-irradiated TN sample was generally well myelinated but there were rare degenerating axons. Mild lymphocytic infiltrate, likely related to MS, was present in $2 / 3$ samples. The three irradiated MS-TN specimens had axon loss, demyelination, myelin debris, and fibrosis. Irradiation at this dose, which was ultimately ineffective for treatment of $\mathrm{TN}$, causes considerable damage to the trigeminal nerve. These data add insight into the changes in a trigeminal nerve due to GammaKnife radiosurgery. 


\section{H.11}

Diagnosing rare neurological disorders with exome sequencing: FORGE Canada

J Warman Chardon (Ottawa)* C Beaulieu (Ottawa) J Schwartzentruber (Ottawa) J Marcadier (Ottawa) D Dyment (Ottawa) S Sawyer (Ottawa) M Geraghty (Ottawa) A Smith (Ottawa) L McDonell (Ottawa) F Consortium (Ottawa) J Majewski (Montreal) D Bulman (Ottawa) K Boycott (Ottawa)

Background: Exome sequencing is a powerful approach targeting protein coding regions of genes that has revolutionized disease gene identification in neurological disorders. FORGE Canada (Finding of Rare Disease Genes) is a national consortium of clinicians and scientists using exome sequencing to identify gene mutations associated with rare disorders. Method: FORGE comprises internationally-recognized Canadian scientists using the infrastructure of the Genome Canada Science and Technology Innovation Centres to sequence human exomes and to identify the molecular etiology of rare genetic disorders. Results: Thus far, 135 of the 187 disorders studied demonstrated neurological features, with 77 genes identified. Exome sequencing has facilitated: (1) the discovery of new neurological disorders, (e.g., Hereditary Spastic Paraplegia type 54); (2) the identification of novel mutations in known genes (e.g., Autosomal Recessive Primary Microcephaly); and (3) the expansion of the phenotype of known disorders (e.g. isolated Primary Dystonia caused by a novel mutation in the ATM gene). Conclusions: Our experience demonstrated immediate and long-term benefits for patients with rare neurological disorders, including molecular diagnosis translating to improved patient care and directed therapy, as well as identifying pathways for further study and potential therapeutic investigation. Canada is emerging as an international leader in the study of rare neurological disease.

\section{ANEURYSMS AND SUbARACHNOID HEMORRHAGE}

\section{I.01}

Reporting the design of the ISAT-II study: a randomized clinical trial comparing surgical and endovascular treatments of ruptured intracranial aneurysms

AE Jack (Edmonton)* TE Darsaut (Edmonton) R Kerr (Oxford) $J$ Raymond (Montréal)

Background: ISAT demonstrated improved one-year clinical outcomes for patients with ruptured intracranial aneurysms treated with coiling compared to clipping. ISAT patients were mostly good grade subarachnoid hemorrhage $(\mathrm{SAH})$ patients with small anterior circulation aneurysms. ISAT results are now commonly extrapolated to patients not studied in the original trial, some who are treated using devices not available at that time. Conversely, many patients are treated by clipping despite ISAT, usually to offer more durable protection from aneurysm recurrences. These practices have never been validated. For many ruptured aneurysm patients the question of which treatment leads to a superior clinical outcome remains unclear. Methods/Design: ISAT-II is a pragmatic, multicenter, randomized trial comparing clinical outcomes for non-ISAT patients with SAH allocated coiling or clipping. Inclusion criteria are broad.
The primary end-point is incidence of poor clinical outcome $(\mathrm{mRS}>2)$ at one year, just as in ISAT. Secondary end-points include measures of safety for several pre-specified subgroups, with efficacy end-points including major recurrences at one-year; 1896 patients (862 each arm plus $10 \%$ losses) are required to demonstrate a significant difference between coiling and clipping, hypothesizing $23 \%$ and $30 \%$ poor clinical outcome rates for coiling and clipping respectively. The trial should involve at least 50 international centres, and take approximately 12 years. Analysis will be by intention-to-treat. ISAT-II is registered: clinicaltrials.govNCT01668563.

\section{I.02}

Decreased rates of perioperative stroke and delayed cerebral ischemia following implementation of a comprehensive neurovascular program

A Algird (Hamilton)* C Martin (Hamilton) DJ Sahlas (Hamilton) D Jichici (Hamilton)

Background: The Neurovascular program at our center was expanded to include $24 / 7$ access to neuroendovascular services with protocols for coiling as the first option for treatment of aneurysmal subarachnoid hemorrhage (aSAH), and a Nurse Practitioner (NP) to perform Transcranial Doppler (TCD) and assist with clinical management. Methods: 205 aSAH patients' charts were reviewed pre and post implementation of the program collecting the following data: demographic, clinical presentation, radiological evidence of perioperative stroke and delayed cerebral ischemia (DCI), clinical evidence of vasospasm, modified Rankin Score (mRS) at hospital discharge, and mortality rate. Results: 100 patients between 20072009 were compared to 105 patients between 2010-12. Incidence of radiological perioperative stroke decreased from $44 \%$ to $22 \%$ $(\mathrm{p}=0.001)$. This corresponded to a practice shift from aneurysmal clipping to coiling. Also, there was a decrease in radiological perioperative stroke rates within the coiling group of patients $(16 \%$ compared with $35 \%, \mathrm{p}=0.04$ ) and a decreased incidence of DCI from cerebral vasospasm (30\% compared with $50 \%, \mathrm{p}=0.04$ ) following implementation of the new program. Conclusions: Implementation of comprehensive neurovascular program for aSAH resulted in a $50 \%$ decrease in the number of perioperative strokes. The decrease in the rate of DCI secondary to cerebral vasospasm in the coiled patients correlated with TCD monitoring and the role of the NP ensuring that the management protocol was followed.

\section{I.03}

PRET Study: patients prone to recurrence after endovascular treatment

\section{J Raymond (Montréal)* D Roy (Montréal)}

In patients with intracranial aneurysms treated with endovascular coiling, angiographic recurrences are often observed. This problem becomes significant in patients with large or recurring aneurysms, which we label "aneurysms with a high Propensity for Recurrence after Endovascular Treatment" (PRET). The PRET RCT thus aims at comparing treatment with hydrogel coils to standard treatment in patients with large aneurysms ( $\geq 10 \mathrm{~mm}$; PRET-1 patients) or in patients with a major recurrence after previous coiling (PRET-2 patients). The primary hypothesis is that the use of hydrogel coils decreases recurrence rate from $50 \%$ to $30 \%$ (range: $40-50 \%$ to 21 - 
$30 \%)$ at 18 months as compared to bare platinum coils. The design is a multi-centre, randomized, controlled trial with concealed allocation, with Data Safety and Monitoring Committee (DSMC) overview. A total of 500 subjects (250 PRET-1; 250 PRET-2) equally divided between the two treatment arms are followed for 18 months. The primary endpoint is recurrence rate, multiply defined; the secondary endpoints include safety measures and overall morbidity and mortality. Recruitment is almost completed with more than 400 (PRET-1: 221; PRET-2: 182) subjects enrolled. Three Interim Safety Analyses were carried out by the DSMC and did not reveal a significant difference between treatment groups on recorded safety measures.

\section{I.04}

FIAT: flow diversion in intracranial aneurysm treatment: a randomized trial comparing flow diversion and best-standardtreatment

TE Darsaut (Edmonton)* D Roy (Montréal) A Weill (Montréal) J Raymond (Montréal)

Large/giant, fusiform or recurrent intracranial aneurysms are increasingly treated with flow diverters (FDs). Although there is growing enthusiasm to use these devices, complications are increasingly reported in series and registries. There is thus an urgent need for an RCT to evaluate FDs in patients presenting with a difficult aneurysm. The FIAT (Flow diversion in Intracranial Aneurysm Treatment) trial aims to compare flow diversion (FD) to Best-Standard Treatment (BST). BST may be: 1) conservative management; 2) coiling with or without stenting; 3) parent vessel occlusion with or without bypass; 4) surgical clipping; 5) registry for FD, when the only treatment alternative is FD for compassionate use. The primary hypothesis is that FD can be performed with an "acceptable" complication rate (15\% morbidity and mortality, modified Rankin Score $>2$ at 3 months), AND increase the number of patients experiencing successful therapy (near complete occlusion of the aneurysm from 75 to $90 \%$ ). 344 subjects will be recruited and followed for 12 months. Follow-up includes two clinical and neurological assessments and vascular imaging at the 12 month follow-up. A pilot phase is underway in Canada and has already enrolled 23 FIAT subjects.

\section{I.05}

The utility of the electrocardiogram in predicting angiographic vasospasm following aneurysmal subarachnoid hemorrhage

GM Ibrahim (Toronto)* $R$ Macdonald (Toronto)

Background: Angiographic vasospasm following aneurysmal subarachnoid hemorrhage ( $\mathrm{SAH}$ ) is a significant cause of morbidity and mortality, with few early predictors. Increased central sympathetic activity may contribute both to angiographic vasospasm and electrocardiographic (ECG) changes after SAH. Here, we determine the association between ECG changes and angiographic vasospasm after SAH. Methods: Exploratory analysis was performed on 413 patients from the CONSCIOUS-1 trial. ECGs were obtained within 24 hours of aneurysm rupture and during the vasospasm risk period. Angiographic vasospasm was assessed using catheter angiography at baseline and 7 to 11 days after SAH. Multivariate logistic regression was used to identify significant associations. Results: The most prevalent finding on ECG both immediately following $\mathrm{SAH}$ and during the vasospasm risk period was QT prolongation (42\% and 25\%, respectively). Prolonged QT interval both at baseline (OR, 1.85;95\% CI:1.00-3.45) and during the vasospasm risk period (OR, 3.53;95\%CI:1.69-7.39) and tachycardia on the baseline ECG (OR:10.83;95\%CI:1.17-100.50) were associated with angiographic vasospasm. Receiver Operator Characteristics curve analysis revealed no difference in predictive value between baseline subarachnoid clot burden and prolonged QT at three days $(\mathrm{p}=0.26)$ and baseline tachycardia $(\mathrm{p}=0.18)$. Conclusions: QT prolongation and tachycardia on ECG were independently associated with angiographic vasospasm after aneurysmal SAH on multivariate analysis.

\section{I.06}

Angiographic outcome of intra-arterial milrinone on cerebral vasospasm after subarachnoid haemorrhage

K Elayoubi (Montréal)* D Lancu-Gontard (Montréal) T Nguyen (Montréal) F Guilbert (Montréal) J Raymond (Montréal) D Roy (Montréal) A Weil (Montréal)

Purpose: The goal of this study was to determine whether there is a change in arterial diameter after intraarterial milrinone infusion for cerebral vasospasm secondary to subarachnoid haemorrhage (SAH). Methods: We retrospectively reviewed the procedure reports, clinical charts, CT and angiograms of patients with symptomatic cerebral vasospasm after SAH. Eight independent radiologists reviewed angiograms of cerebral vessels treated (or not) by intraarterial infusion of milrinone. The arterial diameter assessment was done in a blinded manner. The study group included arteries that received milrinone with or without balloon angioplasty. Controls corresponded to arteries diagnosed with or without vasospasm and that did not receive milrinone. Changes in the arterial diameter were coded as 1 . Changes in arterial diameter were pooled and compared for all reviewers. Results: Twenty-one patients underwent angiograms for symptomatic vasospasm after SAH between October 2004 and August 2006. Nine cerebral arteries treated with intraarterial milrinone and nine control arteries were assessed for the arterial diameter change. Improved arterial diameter was significantly more often seen $(\mathrm{p}<0.0001)$ in arteries after addition of milrinone $(65 / 72,90 \%)$ than in controls $(8 / 72,11 \%)$. Conclusion: Intraarterial milrinone improves angiographic vasospasm after SAH. However, how long this effect persists is still unknown. Further prospective studies are warranted to asses the effect of milrinone on intracranial artery diameter as well as its clinical impact.

\section{I.07}

Familial intracranial aneurysms in Newfoundland and Labrador: updated report

FB Maroun (St. John's)* B Fernandez (St. John's) J Jacob (St. John's) G Murray (St. John's) B Noble (St.John's)

Introduction: It is estimated that $20 \%$ or more of aneurysms are familial. The purpose of this study is to delineate the clinical, epidemiological features of familial and sporadic intracranial aneurysms (ICA) and identify gene(s) that predispose to them. Method: Once ICA patients were identified, a 3-generation pedigree was constructed and the participant was classified familial or sporadic. DNA was collected and molecular study as well as 3D-CT 
scan or MRA were requested. We focused on recruiting familial cases, particularly those with more than two affected relatives. Result: The present cohort includes 86 probands with ICA including 50 familial probands, 34 of their affected relatives and 338 of their unaffected relatives. There are 29 probands with sporadic ICAs and 14 unaffected relatives. In total 558 individuals have been enrolled. CT or MRA has been completed on 437. Of the 117 affected participants, clipping has been performed on 42, coiling on 22. 53 patients are being followed for unruptured aneurysms. Death from $\mathrm{SAH}$ occurred in 4 . There are 8 major families (each with $4-11$ affected individuals) and these will be the focus of future molecular genetic studies. Details of some of these families will be discussed. Conclusion: Identification of patients with familial ICA is important for early treatment, family screening and eventual prevention of subarachnoid hemorrhage.

\section{I.08}

Excellent long-term outcomes in complex intracranial aneurysms treated with PED

E Barber (Saskatoon)* SU Ahmed (Saskatoon) Z Tymchak (Saskatoon) ME Kelly (Saskatoon)

Background: The Pipeline embolization device (PED) is a flowdiverting stent that has been used to treat complex aneurysms not amenable to treatment alternatives. We present a single-centre experience of the long-term efficacy and outcomes of PED placement. Methods: Case data of aneurysms treated with the PED were collected prospectively and pooled for retrospective analysis. Clinical outcomes were converted to modified Rankin scores and imaging was used to determine post-procedure aneurysm filling. Results: 32 PEDs were placed in 16 patients. Patients were followed-up for an average of 20 months. Angiographic cure was achieved in $12 / 16$ patients, and $14 / 16$ scored $\leq 2$ on the modified Rankin scale at most recent follow-up. One patient presenting with SAH died, and a second suffered progressive right-sided weakness leading to hemiplegia. Conclusions: Our experience shows high rates of angiographic cure and favourable clinical outcomes at longterm follow-up, and suggests that the PED is a viable treatment option for complex cerebral aneurysms.

\section{I.09}

Flow diversion of giant curved sidewall and bifurcation aneurysms: very low porosity devices may not produce reliable occlusion in experimental models

TE Darsaut (Edmonton)* F Bing (Strasbourg) A Makoyeva (Montréal) I Salazkin (Montréal) G Gevry (Montréal) J Raymond (Montréal)

Background and Purpose: Flow diverters (FDs) are increasingly used to treat difficult intracranial aneurysms. Giant curved sidewall (cSW) and bifurcation aneurysms present special difficulties that could perhaps be overcome using very low porosity devices. Materials and Methods: Large and giant endwall bifurcation (EwB; $\mathrm{n}=12)$ and $\mathrm{cSW}$ aneurysms $(\mathrm{n}=5)$ were constructed in $17 \mathrm{dogs}$. EwB aneurysms were treated with $48(n=4), 64(n=4)$ or two telescoping 64 wire low-porosity devices $(\mathrm{n}=4)$, while all cSW aneurysms were treated with 64 wire devices. Angiographic results were recorded immediately, at 2-4 and at 12 weeks, immediately before euthanasia. Pathological specimens were photographed and metallic and neointimal closure of the aneurysm ostium measured and scored.
Results: One of twelve EwB and 1/5 cSW aneurysms were occluded at 12 weeks. All other aneurysms were patent. Device-related stenoses occurred in 13/17 animals, hemodynamically significant in two. All branches jailed by the FDs remained patent. For all animals, there was a significant correlation $(\mathrm{P}=0.04)$ between the angiographic scores and the degree of neointima formation (Rho $=$ 0.527 ). Failures of aneurysm occlusion could be explained by leaks or holes, sometimes barely visible, in the neointima over the device, which tended to occur at the level of specific, transition zones within the stent. Conclusion: Low porosity FDs can fail to occlude experimental giant EwB and cSW aneurysms.

\section{I.10}

STAT: stenting in the treatment of large, wide necked or recurring intracranial aneurysms trial

TE Darsaut (Edmonton)* D Roy (Montréal) A Weill (Montréal) J Raymond (Montréal)

In patients with intracranial aneurysms treated with endovascular coiling, angiographic recurrences are often observed. This problem becomes significant in patients with large, recurring, or wide necked aneurysms. Stenting combined with coil occlusion presumably modifies intra-aneurysmal hemodynamics and leads to a decrease in recurrence rate. The STAT (Stenting in the Treatment of large, wide necked or recurring intracranial Aneurysms Trial) RCT thus aims at comparing coiling versus coiling plus stenting in patients with aneurysms prone to recurrence, i.e. large aneurysms $(\geq 10 \mathrm{~mm}$; STAT$1)$, recurring aneurysms after previous coiling (major recurrence; STAT-2), or wide-necked aneurysms (aspect ratio $<1.5$; STAT-3). The primary hypothesis is that the use of stenting in addition to coiling decreases the recurrence rate from $33 \%$ to $20 \%$ at 12 months. Six hundred subjects will be enrolled and followed for 12 months. The interventions are : coiling (any type); OR the addition of stenting to coiling (any stent). Follow-ups include clinical and neurological evaluation and, for the 12 months follow-up, angiographic imaging of the aneurysm. The primary endpoint is recurrence rate, multiply defined; the secondary endpoints include safety measures, overall morbidity and mortality and incidence of in-stent stnenosis $(>50 \%)$. A pilot phase is underway in Canada and has already enrolled 9 STAT subjects.

\section{I.11}

2013 update on the Canadian Unruptured Endovascular vs Surgery (CURES) trial

\section{TE Darsaut (Edmonton)* M Findlay (Edmonton) J Raymond (Montreal)}

Background: The best treatment for patients with unruptured intracranial aneurysms (UIA) remains uncertain. Surgical clipping is widely considered to provide more consistent and permanent aneurysm exclusion and better long-term protection from hemorrhage but may result in greater morbidity than endovascular treatment. A randomized comparison of the two treatments has not been done. Purpose: To compare anatomical results, treatment morbidity \& mortality, and long-term clinical outcome of surgical clipping versus endovascular coiling of intracranial aneurysms in a randomized controlled trial. Methods: So far, in Canada, 53 patients with UIAs have been randomized. The available anonymized data will be presented at the conference. Conclusion: CURES is a CIHR- 
funded, two-phase RCT comparing angiographic and clinical outcomes. The lead-in phase aims to verify superior anatomical results of clipping and determine its risks. Phase II will compare clinical outcomes including overall re-treatment rates and bleeding at five years.

\section{Pediatrics}

\section{J.01}

The metabolic phenotype guiding the discovery of treatable intellectual disability genes

$C$ van Karnebeek (Vancouver)* $R$ Salvarinova (Vancouver) $C$ Shyr (Vancouver) G Sinclair (Vancouver) A Lehman (Vancouver) A Waheed (St Louis) W Sly (St Louis) W Wasserman (Vancouver) S Sylvia (Vancouver)

Introduction: Intellectual disability (ID) is a lifelong, debilitating condition affecting $2.5 \%$ of children and adults worldwide. Our TIDEX project aimed to identify novel, potentially treatable ID genes employing the utility of the metabolic phenotype. Methods: Criteria were applied to select patients for NGS: patient with unexplained, Mendelian ID plus metabolic abnormalities. Whole exome sequencing was performed for trio's with customized bioinformatics and subsequent validation. Results: In seven families meeting the selection criteria, we discovered five new genes, including a new treatable disorder Mitochondrial carbonic anhydrase VA (CA-VA) deficiency identified in two nonconsanguineous siblings with neonatal lethargy, hyperammonemia, hyperlactatemia, hypoglycemia. CA-VA deficiency impairs bicarbonate provision to four mitochondrial enzymes with a central role in the urea cycle and intermediary metabolism. CA-VA deficiency is amenable to preventive and emergency treatment, and expands the number of treatable IDs. The other discovered genes (various phases validation) include a nuclear mitochondrial disease, biotin responsive, neuronal arborization, early endosomal recycling genes. Conclusions: Our high success rate $(>70 \%)$ emphasizes the importance of the metabolic phenotype for gene discovery using NGS technologies. Advantages include: facilitation candidate gene hypothesis, validation causality identified variants, and targets for treatment. Most important, such discoveries can be directly translated into improved patient outcomes.

\section{J.02}

Evaluating the exposure of radiation from CT scans of children presenting with primary headache

$R$ Gandhi (Ottawa)* E Lewis (Ottawa) J Evans (Ottawa) E Sell (Ottawa)

Objective: To determine the frequency of computed tomography (CT) scanning ordered by a range of medical practioners for pediatric patients presenting with migraine headaches. Background: Headaches are a common problem in the pediatric population. In 2004, The American Academy of Neurology (AAN) developed guidelines on neuroimaging for headache. The medical-legal climate may be causing physicians to disregard these guidelines. Methods: A retrospective chart review was conducted at the Children's Hospital of Eastern Ontario (CHEO), a tertiary care center in Ontario. Records from 2004-2010 were collected for pediatric patients referred to the outpatient neurology clinic at CHEO with 'headache' as the primary complaint. Results: Thirty-four percent (34/99) of patients presenting with normal neurologic exams had undergone a CT scan. Zero percent (0/34) of CT scans showed significant findings, of which zero percent $(0 / 34)$ of these CT scans changed the headache diagnosis or management. Thirty-eight percent (13/34) of CT scans were ordered by neurologists, thirty-eight percent $(13 / 34)$ by community physicians, and twenty-four percent (8/34) by CHEO emergency physicians. Conclusion: Physicians are performing unnecessary CT scans on children presenting with primary headaches, despite well established AAN guidelines regarding neuroimaging. The reality of the medical-legal climate in North America encourages physicians to over-investigate, unnecessarily increasing the risk of adverse events from radiation exposure to pediatric patients.

\section{$\mathbf{J . 0 3}$}

MRI findings in infants with infantile spasms after neonatal hypoxic-ischemic encephalopathy

D Gano (Vancouver)* MA Sargent (Vancouver) SP Miller (Toronto) $M B$ Connolly (Vancouver) P Wong (Vancouver) HC Glass (San Francisco) KJ Poskitt (Vancouver) V Chau (Vancouver)

Background: Neonatal hypoxic-ischemic encephalopathy (HIE) is a common cause of infantile spasms (IS). The purpose of this study was to evaluate the predominant pattern of brain injury and the anatomic areas of injury in children with IS following neonatal HIE. Methods: A nested case-control of IS in children with term neonatal HIE was performed. All patients had T1/T2-weighted MRI with diffusion-weighted imaging on day 3 of life. Using a validated scoring system, the predominant pattern of injury was prospectively classified as: normal, watershed, basal ganglia/thalamus (BG/T), total, or focal-multifocal. Two study investigators scored additional anatomic areas of injury (cortical extent, brainstem, hypothalamus) blinded to the outcome. The pattern of injury and anatomic areas of injury were compared between cases with IS and randomly selected controls. Results: Eight patients who developed IS were identified among a cohort of 176 term newborns with HIE ( 4.5\%). There were no significant differences in the perinatal and neonatal course between newborns who developed IS and controls. The development of IS was significantly associated with $\mathrm{BG} / \mathrm{T}$ and total brain injury $(\mathrm{P}=.001)$, extent of cortical injury $\geq 50 \%(\mathrm{OR}=11.67,95 \% \mathrm{CI}=1.1$ $158.5, \mathrm{P}=.01)$, injury to the midbrain $(\mathrm{OR}=13,95 \% \mathrm{CI}=1.3-172$, $\mathrm{P}=.007)$ and hypothalamic abnormalities $(\mathrm{P}=.01)$. Conclusions: The development of IS after HIE is associated with extensive brain injury on neonatal MRI, particularly when the midbrain is involved.

\section{J.04}

\section{Malignant infarction of the middle cerebral artery in children}

AV Andrade (Toronto)* I Yau (Toronto) G deVeber (Toronto)

Background: Large hemispheric strokes have a high risk of fatal cerebral edema. This syndrome has been described as "Malignant infarction of the middle cerebral artery" (MIMCA), this complication is commonly seen in adults. The description of this syndrome in the pediatric population is scarce. The goal for the study is to identify possible clinical, radiological and electrographic features associated with MIMCA syndrome in children. Patients and Methods: We reviewed retrospectively, patients with a definite diagnosis of arterial ischemic stroke of the middle cerebral artery 
(MCA) that developed cerebral edema, and were evaluated for hemicraniectomy. Results: We identified 117 children with stroke at the Hospital for Sick Children, from January 2008 to September 2012. 68 (58\%) had arterial ischemic stroke (AIS), and 30 (44\%) had MCA AIS. 8 patients developed MIMCA. 6/8 were boys and 2/8 were girls. Mean age was 10 years (range from 3 to 15 ). The most common etiology was cardioembolic (5/8).6/8 had a PedsNIHSS score greater than $10.5 / 8$ presented with seizures, $8 / 8$ had hyperdense MCA sign on initial non contrast CT head. Discussion: Although MIMCA is rare in children, its early recognition and prompt surgical management appears to be related with a better outcome. Hyperdense MCA sign, seizures and a score equal or higher than 10 at the PedsNHISS are common features in children with MIMCA.

\section{J.05}

Safety and tolerability of TMS and rTMS in a pediatric clinical trial

$T$ Rajapakse (Calgary), O Damji (Calgary) J Roe (Calgary) A Kirton (Calgary)*

Background: Transcranial magnetic stimulation (TMS) can define neurophysiology while repetitive TMS (rTMS) may modulate neuroplasticity. Applications in the developing brain are limited. Methods: PLASTIC CHAMPS is a randomized, blinded, factorial clinical trial of rTMS and constraint therapy in children aged 6-18 years with perinatal stroke-induced cerebral palsy. Thirty five children (mean 11years) attended a 2-week motor learning camp, randomized to daily non-lesioned M1 inhibitory $(1 \mathrm{~Hz}, 1200$ stimulations) rTMS or sham. TMS neurophysiology was assessed pre- and post-camp. Safety outcomes were: 1) decreased affected hand function (Assisting Hand Assessment, AHA; Melbourne Assessment; MA) 2) decreased unaffected hand function (grip, pinch strength (GS/PS)) and 3) Pediatric TMS Tolerability Scales at 4 timepoints. Differences were compared between groups and over time. Results: TMS and rTMS were well tolerated with no drop outs or serious adverse events. Affected hand function did not decrease (stable AHA, improved MA) including children with ipsilateral projections. Unaffected hand function was stable. Side effects were mild with headache most common (43\% 1st TMS) and decreasing (20\% last TMS). Headache was rare during rTMS. Conclusions: TMS trials are safe in children with perinatal stroke. Headaches are common but mild. Inhibitory rTMS does not negatively impact hand function on either side.

\section{J.06}

Serial diffusion imaging of cerebral diaschisis in childhood arterial ischemic stroke

E Williams (Calgary), M Dowling (Dallas) R Ichord (Philadelphia) A Kirton (Calgary)*

Background: Acute corticospinal diffusion MRI (DWI) correlates with motor outcome in childhood arterial ischemic stroke (AIS). In neonatal AIS, DWI detects changes in other remote but connected brain structures (diaschisis). We hypothesized that cerebral diaschisis is measureable in childhood AIS and associated with outcome. Methods: This sub-study (Validation of the PedNIHSS) prospectively enrolled 112 AIS children imaged at diagnosis (MR1) and 5-14 days (MR2). Inclusion criteria: unilateral
AIS, MR1/2 DWI, and 12 month Pediatric Stroke Outcome Measure. Diaschisis was localized and quantified using validated ImageJ software. Diaschisis scores corrected for stroke volume were compared to outcome. Results: Nineteen children (53\% male, median 8.1 years) had median MRI times of 21 and 168 hours. Diaschisis was common and evolved over time; observed in $1(5 \%)$ on MR1 but $8(42 \%)$ by MR2. Thalamic and callosal diaschisis were common $(5 / 8,63 \%)$. Children with diaschisis tended to be younger $(\mathrm{p}=0.14)$. Diaschisis scores were associated with poor overall outcome $(p=0.08)$ with strongest correlations to non-motor outcomes $(\mathrm{p}=0.05)$. Corticospinal DWI correlated with poor motor outcome $(\mathrm{p}=0.004)$. Reliability was excellent $(\varrho=0.99)$. Conclusions: Diaschisis is common in childhood AIS and should not be mistaken for new infarction. Correlation to non-motor outcome suggests a possible imaging biomarker of network injury.

\section{J.07}

Glycyl-tRNA synthetase (GARS) mutations causing systemic mitochondrial disease

HJ McMillan (Ottawa)* P Chakraborty (Ottawa) S Lee (Ottawa) J Schwartzentruber (Montreal) A Smith (Ottawa) DE Bulman (Ottawa) C Beaulieu (Ottawa) F (Ottawa) J Majewski (Montreal) KM Boycott (Ottawa) MT Geraghty (Ottawa)

Background: Aminoacyl-tRNA synthetases (ARSs) are essential for the first step of protein translation by linking a specific amino acid to its corresponding tRNA. Glycyl-tRNA synthetase (GARS) is a bifunctional protein active within cytoplasm and mitochondria. Dominant mutations in GARS have been linked to CMT2D and distal spinal muscular atrophy. We report a case of a girl who presented at six years (yrs) old with exercise-induced myalgia. An echocardiogram showed left ventricular non-compaction with normal function. She had persistent elevation of blood lactate and alanine. Her initial neurological exam, NCS/EMG and muscle biopsy (including respiratory chain enzyme and mtDNA sequencing) were normal. MRI brain (10 yrs old) revealed mild periventricular leukomalacia. Repeat evaluation (12 yrs old) revealed mild distal foot weakness and a low right common peroneal nerve CMAP amplitude. Method: Exome sequencing revealed compound heterozygous mutations within each GARS allele. Results: One affected allele was inherited from each parent. The paternally inherited mutation (c.1904C>T; p.S635L) has been previously linked to CMT2D and the father was shown to have mild sensorimotor polyneuropathy. The maternally inherited allele (c.1787G >A; p.R596Q) is previously unreported but occurs at a highly conserved site and is predicted to be deleterious. Conclusions: The clinical phenotype of this girl expands our understanding of GARS mutations and when present in a compound heterozygous state can present with a mitochondrial disease phenotype.

\section{J.08}

\section{CSF leak after intradural spinal surgery in children}

CC Gillis (Vancouver)* V Liu (Vancouver) A Singhal (Vancouver) D Cochrane (Vancouver) P Steinbok (Vancouver)

Cerebrospinal fluid (CSF) leakage is a complication of intradural spinal surgery and is associated with poor wound healing and infection. The incidence of CSF leak is reported at $\sim 16 \%$ in adults, 
but little information is available in children. This study was a retrospective chart review of 638 intradural spinal operations at B.C. Children's Hospital. CSF leak was defined as pseudomeningocele or CSF leak through incision. Primary operations to untether lipomyelomeningoceles, myelomeningocele/ meningocele closure, and Chiari decompressions were excluded. CSF leaks occurred in $7.1 \%$, with $3 \%$ having overt CSF leaks through skin (OCSF leak). CSF leaks, specifically OCSF leaks, were associated with postoperative wound infection ( $\mathrm{p}=0.0016) .16$ of 45 cases of CSF leak required re-operation. The type of suture, anatomic site, or use of fibrin glue did not affect CSF leak rates. Previous spinal surgery $(\mathrm{p}<0.0001)$, use of dural graft $(\mathrm{p}=0.0043)$, method of dural suturing $(\mathrm{p}=0.0023)$, and procedure performed $(\mathrm{p}<0.001)$ were associated with CSF leakage. Patients with CSF leak were older than those without leak (98 vs. 72 months, $\mathrm{p}=0.002$ ). Our results provide evidence on intraoperative factors that may predispose to CSF leaks after spinal intradural surgery and may help guide surgical practice. This study confirms that the pediatric population shares similar risk factors to the adult population. Further research is needed to explain how specific factors are associated with CSF leaks.

\section{J.09}

Paired associative stimulation to understand neuroplasticity in children

O Damji (Calgary)* J Roe (Calgary) S Shinde (Calgary) $O$ Kotsovsky (Calgary) A Kirton (Calgary)

Introduction: Transcranial magnetic stimulation (TMS) noninvasively assesses neurophysiology. Paired associative stimulation (PAS) combines peripheral sensory stimulation with TMS over the primary motor cortex (M1). PAS induces rapid, reversible, topographically specific increases in M1 excitability consistent with NMDAR-dependent long-term potentiation. PAS has not been studied in children. Methods: Healthy right-handed children (6-18 years) were recruited. Median nerve stimulation was delivered $25 \mathrm{~ms}$ prior to suprathreshold left M1 stimulation. Primary outcome was change in motor evoked potential (MEP) amplitude from baseline to five time-points post-PAS (0/15/30/45/75 minutes). Effects were categorized as definitive, possible, or no effect. Secondary outcomes included stimulus response curves (SRC), and safety and tolerability evaluations. Results: Of 15 children (9 male, mean age 12 years), 8 $(53 \%)$ showed definitive PAS effect ( 2 possible, 5 no effect). Maximal effect was seen at 0 minutes in 5 and 15 minutes in 3 . Mean SRC slope increased across all subjects $(\mathrm{p}=0.05)$. Tolerability scores were favorable with no adverse events. Conclusions: PAS appears safe and tolerable in children. Frequency of PAS effects seems higher in children $(>50 \%)$ than adults. SRC curves may be more sensitive at detecting PAS effects. PAS may provide insight on developmental plasticity and therapeutic targets in children with cerebral palsy and motor disorders.

\section{J.10}

EEG biomarkers of poor neuropsychological outcome following perinatal stroke

A Mineyko (Calgary)* B Brooks (Calgary) H Carlson (Calgary) L Bello-Espinosa (Calgary) A Kirton (Calgary)

Background: Perinatal stroke (PS) causes most hemiplegic cerebral palsy and often intellectual disability. Evidence suggests children with arterial lesions have dichotomized neuropsychological outcomes. We hypothesized neuropsychological outcomes correlate with pathological changes on EEG after PS. Methods: Retrospective analysis of Calgary Pediatric Stroke Project data ( $>3$ years-of-age, arterial PS, available neuropsychological evaluation) was combined with prospective data (post-neonatal EEG with sleep). Continuous epileptiform discharges in slow wave sleep (CDSS) for discharges in $>75 \%$ of recording were analyzed by blinded EEG review. Severe neuropsychological outcomes consisted of intellectual scores <2SD of age mean, or impairment preventing testing. Results: Seventyone children with arterial PS were evaluated. Ninety-three EEGs were available for 48 patients. Seven (15\%) were showed CDSS. Twenty-nine neuropsychological test results were available. Of the seven with CDSS, all had clinical epilepsy and severe neuropsychological outcomes. CDSS correlated with severe neuropsychological outcome $(\mathrm{p}<0.001)$. Eighteen of the remaining 22 children $(82 \%)$ had favorable intellectual outcome ( $\geq 2$ SD of the mean). Conclusions: CDSS is a potential EEG biomarker of severe intellectual impairment in children with PS. Integration of EEG findings may improve predictive models of neuropsychological outcomes. Identifying abnormal rhythms early in development may represent a new therapeutic target.

\section{J.11}

Systematic screening study for treatable disorders in intellectual disability

$C$ van Karnebeek (Vancouver) T Murphy (Vancouver) $M$ Demos (Vancouver) $K$ Selby (Vancouver) $C$ Vann (Vancouver) $R$ Salvarinova (Vancouver) Y Lillquist (Vancouver) $G$ Horvath (Vancouver) $G$ Sinclair (Vancouver) $H$ Vallance (Vancouver) $M$ Connolly (Vancouver) A Lehman (Vancouver) M Patel (Vancouver) $S$ Stockler (Vancouver)*

Background: Intellectual disability (ID) is a debilitating disorder affecting 2-3\% of patients worldwide. Inborn errors of metabolism (IEM) currently constitute the only group of genetic defects amenable to causal therapy. Early diagnosis prevents or minimizes brain damage. Our literature review identified 81 such treatable IEM; although evidence is limited, therapies are often effective, safe, accessible. Methods: We translated this knowledge into the TIDE diagnostic protocol: The 1st tier comprises metabolic screening tests in blood/urine with potential to identify $62 \%$ of treatable IDs. The second tier focuses on remaining disorders, requiring 'single test per disease' approach. A freely available App (www.treatable-id.org) supports the protocol. Results: In our tertiary care institution one year implementation of this protocol identified treatable IEMs in $>5 \%$ of 210 ID patients. Further analysis comparing these patients to those diagnosed in our hospital between 2000-2009 revealed that the TIDE protocol reduced 'time to diagnosis' by 6 months (range 1-50 months) as well as costs of unnecessary testing ( $>1500$ - per patient). Conclusions: Our protocol for treatable forms of ID has proven effective in terms of increasing the diagnostic yield and reducing costs and diagnostic delay. Improved cognitive functioning allows the patient to reach full potential and avoids unnecessary burden to society. These results provide the evidence to support a new practice for ID patients: prioritize for the treatable ones! 


\section{Poster Presentations}

\section{EPILepsy (EEG, Basic Science, IMAGING, NEUROLOGY AND EPILEPSY SURGERY)}

\section{P.001}

\author{
CJD-like periodic EEG discharges in VGKC-complex antibody \\ encephalitis
}

$M$ Savard (Québec)* S Gosselin-Lefebvre (Québec) RJ Laforce (Québec) A Guillemette (Québec) M Geschwind (San Francisco) SR Irani (San Francisco) P Gould (Québec)

Background: VGKC-complex antibody encephalitis is a treatable, likely antibody-mediated condition, which has been previously reported to clinically mimic Creutzfeldt-Jacob disease. Clinical and radiological clues have been suggested to help distinguish these diseases. However, the periodic discharges classically associated with sporadic CJD have never been reported in association with VGKC-complex antibody encephalitis. Methods: Case report. Results: A 76-year-old male was transferred to a tertiary neurology center with a six-month history of weight loss, cognitive disturbances and generalized weakness. He later had two generalised seizures and developed a severe encephalopathy, requiring mechanical ventilation. Generalised periodic discharges every 1-2 seconds over slowed background were found on EEG, and MRI showed cerebellar and bifrontal cortical T2/FLAIR/DWI hypersignal without restricted diffusion on ADC mapping. Extracerebral PET-scan was negative. Despite administration of steroid/IVIG/PEX, the patient died after life support withdrawal. Brain autopsy revealed arachnoid and white matter perivenular mononuclear infiltrates, with increase in neocortical microglial cells but no significant spongiosis. The VGKC-complex antibody result was $336 \mathrm{pmol} / \mathrm{L}$ (normal: 0-31), reported three months after death. Conclusion: This is the first reported case of VGKC-complex antibody encephalitis associated with periodic EEG discharges, which brings further confound to the differential diagnosis of Creutzfeldt-Jakob disease and autoimmune encephalitis.

\section{$\mathbf{P . 0 0 2}$}

Ketamine usefulness in refractory status epilepticus: a retrospective multicentric study

S Gosselin-Lefebvre (Québec)* A Rabinstein (Rochester) A Rossetti (Lausanne) M Savard (Québec)

Background: Super refractory status epilepticus (SRSE) is a lifethreatening condition, with no established optimal treatment options. Because of the marked increase of gluatmatergic transmission in animal models, an NMDA receptor antagonist such as ketamine could be beneficial. Methods: Between 2004 and 2011, we identified nine consecutive patients with SRSE who were treated with ketamine at three tertiary neurological centers. Results: Median age was 35 years (range 18-78 years) and median number of medications received before ketamine was 8 (range 5-11). Median time before initiation of ketamine was 12 days (range 6-25 days). Median maximal dose was $5 \mathrm{mg} / \mathrm{kg} / \mathrm{h}$ (range $2-15 \mathrm{mg} / \mathrm{kg} / \mathrm{h}$ ). In 4 patients, frank EEG improvement was observed, 3 had modest response, and no effect was observed in 2. There was no direct correlation between ketamine dose and EEG response. Ketamine permanently controlled SRSE in only 1 case. No major side effects were noted. Clinical outcomes were variable; 5 patients died, 1 remained disabled, and 3 had favorable recovery. Conclusion: Although ketamine can result in electrographic improvement in some patients with SRSE, it did not significantly influence the clinical outcome. The long delay before treatment initiation may have limited its usefulness. Earlier administration of ketamine in SRSE should be examined.

\section{P.003}

Acute porphyria presenting as epilepsia partialis continua: a report of two cases

K Leduc (Québec)* T Yen Tran (Montreal) D Nguyen (Montreal) $N$ Dupré (Québec) D Rivest (Lévis) M Savard (Québec)

Background: The porphyrias are a defect in the biosynthesis of haem wich can be associated with different neurologic symptoms during acute attacks such as peripheral neuropathy, mental disturbance and seizures. So far, there has only been a few case reports of status epilepticus, none of which were of epilepsia partialis continua (EPC). Methods: Case series. Results: Case 1 is a 30 -year-old male who came to the emergency room for a convulsive status epilepticus. Case 2 is a 49 -year-old male who first presented a tonic-clonic seizure. Both evolved to EPC over the next days. EPC persisted despite several antiepileptic drugs trials. Diagnosis of hereditary coproporphyria was confirmed by high level of urine, fecal and serum porphyrins in both and by genetic testing in one. The first patient died a month and half after admission from brain oedema. Over the last three years, the second patient has continued to present non-disabling EPC and has had four tonic-clonic seizures associated with alcohol consumption. Conclusion: Acute porphyrias should be included in the differential diagnosis of new onset status epilepticus, including epilepsia partialis continua. Their recognition is important as it modifies significantly patient management, since many anticonvulsants are porphyrogenic.

\section{$\mathbf{P . 0 0 4}$}

Five year experience of a new adult epilepsy program in Canada. The Saskatchewan experience

JF Tellez-Zenteno (Saskatoon)* A Wu (Saskatoon) M Vrbancic (Saskatoon) N Lowry (Saskatoon) F Moien-Afshari (Saskatoon) D Dash (Saskatoon) K Watherhouse (Saskatoon) L HernandezRonquillo (Saskatoon)

Background: There is no doubt about the benefits of surgical programs for patients with intractable epilepsy. We analyzed the experience of a new adult epilepsy surgical program in Canada. Methods: The adult epilepsy program in Saskatchewan has been operating since 2007. The program has the following human resources; three epileptologist, two neurosurgeons, one nurse, and a 
neuro-psychologist. The program has five registered technologist and one video-EEG telemetry bed. Results: The program has performed 158 video-EEG telemetries in five years. Thirty five percent of patients were identified as candidates for epilepsy surgery without intracranial investigation, $21 \%$ had non-epileptic events and $15 \%$ were candidates for epilepsy surgery but needed intracranial recording. In the rest of patients the video-EEG was useful to classify the syndrome. Fifty two patients have had epilepsy surgery in Saskatoon. 41 patients had temporal lobectomies, 8 extratemporal resections and 2 callosotomies. Forty nine patients have had at least one year of follow up after surgery, and $71 \%$ have reached a seizure free status. The program also has a single seizure clinic, provides vagus nerve stimulation and ketogenic diet and soon will have a transition clinic. Conclusions: The results of the Saskatchewan epilepsy program are in line with the international standards in the first years of operation. The access for epilepsy surgery has been improved in the province of Saskatchewan

\section{P.005}

\section{Refractory spike wave after prolonged seizure freedom}

\section{$F$ Moien-Afshari (Saskatoon) G Hunter (Saskatoon) CB Gervais (Saskatoon)*}

Background: We report a case of refractory Non-Convulsive Status Epilepticus (NCSE) in a patient previously seizure-free on Carbamazepine for 16 years. Methods: Case report Results: A 78year-old Caucasian male with primary generalized epilepsy, diagnosed at 52. His seizures were well controlled for many years on Carbamazepine monotherapy. In April 2012 he had a right total knee replacement that became infected, and was admitted to hospital with sepsis in June 2012. After stabilization, he was discharged on longterm outpatient Clindamycin, which was switched to CoTrimoxazole in 4 months and later discontinued. He subsequently had seizure recurrence in August 2012, after being seizure free for years. He had more than a dozen seizures in August, presenting as prolonged, refractory Non-Convulsive seizures. Several events were recorded on EEG telemetry showing generalized polyspike-andwave discharges while the patient simultaneously sustains a simple conversation with the examiner and moves all limbs with dexterity. MRI brain and lab investigations including serum and CSF routine parameters as well as tetrahydrofolate, B6, organic and amino acids were unremarkable. He failed several AEDs and is currently managed on Levetiracetam, Phenobarbital and Carbamazepine. This case is notable for the patient's long-standing seizure-free interval when managed only on Carbamazepine, which defies conventional logic for the treatment of generalized epilepsy. In addition, it indicates long-lasting brain irritability after prolonged antibiotic treatment.

\section{P.006}

\section{Hippocampal spike-and-wave discharges in a rat model of typical absence seizures}

\section{J Arcaro (London) S Mirsattari (London)* SL Leung (London)}

Typical absence seizures are characterized by bilateral $3 \mathrm{~Hz}$ spike-and-wave discharges (SWDs) in humans. Both typical and atypical absence seizures are generated by the interconnected circuitry of the thalamus and cortex. A fundamental distinction is a role of the hippocampus in atypical absence seizures, which is not thought to be involved in typical absence seizures due to the lack of evidence of SWD expression in the hippocampus of animal models. Electroencephalography (EEG) was used to characterize neural activity between the thalamus, cortex and the hippocampus in the gamma-butyrolactone (GBL) rat model of typical absence seizures. Rats $(n=8)$ were surgically implanted with depth electrodes in the medial parietal cortex, frontal cortex, visual cortex, hippocampus and ventrolateral thalamus. Following a week of recovery, rats were injected with $200 \mathrm{mg} / \mathrm{kg}$ GBL intraperitoneally and EEG was recorded continuously for 2 hours. Within 9 minutes of injection, rats became completely immobile with a vacant stare. There was a simultaneous reduction in frequency from a 5-6 Hz burst pattern to continuous at 4-6 Hz with an increase in power. SWDs were recorded from all electrodes with the highest amplitude observed in the hippocampus. The expression of SWDs in the hippocampus demonstrates an interaction between the thalamocortical and hippocampal circuitry and suggests an important link between the hippocampus and typical absence seizures.

\section{P.007}

\section{Childhood onset non-progressive Rasmussen's encephalitis}

$P$ Rizek (London)* C Cheung (London) RS McLachlan (London) B Hayman-Abello (London) DH Lee (London) RR Hammond (London) SM Mirsattari (London)

Background: Rasmussen's encephalitis (RE) is an inflammatory neurological disease that typically manifests with medically intractable partial seizures and a progressive clinical course including unilateral cerebral atrophy, hemiparesis, and cognitive impairment. Since its first description, a number of atypical features are reported that have widened the clinical spectrum of RE. Our goal is to describe patients with pathology-proven RE found to have a non-progressive course on long followup. Methods: Four patients who were referred to our Comprehensive Epilepsy Program at London Health Science Centre in London, Ontario, were diagnosed with RE on a pathological basis after epilepsy surgery to treat their partial onset seizures. Results: None of our four cases followed the typical course of RE despite their childhood onset seizures between ages 2-12 years. One was preceded by a mild head trauma and fever at the onset. None had epilepsia partialis continua (EPC). Their longterm followup revealed a non-progressive form of the syndrome with respect to the neurological examination, EEG, MRI, and neuropsychological findings. Conclusions: These cases extend the spectrum of childhood-onset intractable epilepsy with chronic encephalitis to include non-progressive variants of RE. The absence of EPC may be a prognostic indicator of a non-progressive course.

\section{P.008}

\section{Inter-temporal seizure propagation}

\section{H Abualela (London) ML Jones (London)* WT Blume (London)}

Identified routes of inter-temporal seizure propagation depend heavily on sites of invasive electrode implantation. Studying patients with adequate bi-temporal and bi-frontal subdural electrode coverage, thus encompassing all published proposed routes, we found a variety of propagation pathways. Fifteen (15) electroclinical seizures originated unilaterally in a mesial temporal lobe and propagated to the contralateral temporal lobe among eleven (11) patients with such ictal spread studied. The following 3 routes to the 
contralateral temporal region appeared: 1) direct mesial temporalmesial temporal in $6(40 \%), 2)$ mesial temporal-frontal-temporal in $4(27 \%)$, and 3) mesial temporal - lateral temporal - temporal in 5 (33\%). Median latencies between seizure onset and propagation to the contralateral temporal lobe were: 1) direct: $4.75 \mathrm{sec}, 2)$ via frontal $28.5 \mathrm{sec}$, and 3) via lateral temporal: $20.5 \mathrm{sec}$. Our findings suggest that at least three possible routes for contralateral temporal propagation exist. Additionally, the longer latencies through frontal and lateral temporal routes indicate that more than contralateral temporal receptiveness is requisite for some homotopic ictal involvement.

\section{P.009}

Clinical characteristics and prognosis of autoimmune diseaserelated epilepsy

M Jones (London)* A AlQassmi (London) S Mirsattari (London) J Burneo (London) R McLachlan (London)

There is a now well-established link between epilepsy and autoimmune processes, particularly with respect to certain neuronal autoantibodies. A sizeable subset of patients in this category is typically refractory to anti-seizure drug regimens and requires immunosuppressive therapy. In order to characterize the electroclinical features of such patients, we interrogated our database searching for patients with autoantibody-related epilepsy. Seventeen patients were identified, mean age was 46 years (range 20-72), and 11 patients were female. Four patients presented with status epilepticus. EEG recordings in the majority of the patients showed multifocal abnormalities, primarily epileptiform activity in the temporal lobes. Only 23\% had MRI anomalies consistent with mesial temporal sclerosis. The majority of patients demonstrated a satisfactory response to anti-seizure medications only and did not require immunosuppressive therapy. Recognition of underlying autoantibodies in patients with late onset epilepsy is paramount. A significant proportion of these patients respond favourably to antiseizure medications alone, but a relatively low threshold for use of immunosuppression is generally warranted.

\section{History, EduCATION}

\section{P.011}

Program directors' and residents' perceptions of pediatric neurology training and manpower in Canada

\section{A Doja (Ottawa)* C Clarkin (Ottawa) $S$ Whiting (Ottawa) M Moharir (Toronto)}

Pediatric neurology trainee numbers have grown considerably in Canada resulting in the number of pediatric neurology graduates outpacing the current number of available positions for academic pediatric neurologists. The purpose of this study is to seek the opinion of pediatric neurology program directors (PD's) and trainees regarding possible solutions to this issue. Two semi-structured focus groups occurred in June 2012 involving current and former PD's, and current pediatric neurology trainees The focus groups explored the participants' perceptions of child neurology manpower issues in Canada and possible solutions. Qualitative content analysis was used to analyze the data and identify major themes. Major themes emerging from both focus groups included the emphasis on community pediatric neurology as a viable option for trainees, including the need for community mentors; the recognition of the needs of underserviced areas; and the establishment of academic positions for community preceptors. The need for career mentoring and support structures during residency training was also discussed. Moreover, PDs and residents gave suggestions on reducing the current oversupply of trainees in Canada, including limiting the number of trainees entering programs, as well as for the creation of a long-term vision of child neurology in Canada.

\section{P.012}

Building on in-class neurosciences: evaluation of knowledge during a clinical rotation

CS Casserly (London)* LM Mai (London)* SL Venance (London)*

Background: Canadian medical students undergo preclinical neurosciences education with the goal of building on this knowledge during medical clerkship. Our objective was to assess core neuroscience knowledge before and after a clinical experience. Methods: A quiz consisting of 6 clinical vignettes and 30 multiplechoice questions was administered at the beginning and end of a two week clinical neurology rotation to senior medical students $(n=50)$. Results: Overall there was no significant difference between pre-test and post-test scores (mean scores 17.8 \pm 3.5 and 18.8 \pm 3.8 respectively, $\mathrm{p}=0.20)$. Individual interval improvement was $1.1 \pm 3.2$. Forty participants completed both tests with $60 \%(n=24)$ improving, $28 \%(n=11)$ worsening and $12 \%(n=5)$ unchanged. A control group of six neurology residents scored $23.2 \pm 1.9(\mathrm{p}=0.03)$. There were no differences between first and second year medical clerkship students, or between Western students $(n=28)$ and other Canadian $(n=15)$ or international $(n=5)$ schools. Conclusions: The lack of improvement was disappointing. Explanations include use of negatively phrased questions (4), and questions targeted beyond the expected scope of medical students (5) (e.g. management and medication) rather than recall of basic knowledge. Immediate feedback on pre-test performance to identify gaps coupled with coaching by teachers to consolidate and apply knowledge may improve performance.

\section{P.013}

Canadian trends in the management of intracranial aneurysms: implications for the education of the next generation of neurosurgeons

NS Alshafai (Toronto)* M Cusimano (Toronto) O Falenchuk (Toronto)

Background: The publication of the International Subarachnoid Aneurysm Trial rapidly changed the management of patients with subarachnoid hemorrhage. The present and perceived future trends of aneurysm management have significant implications for patients and how we educate future cerebrovascular specialists. Objective: To determine present competencies, expectations from graduating neurosurgical residents, implications and the geographical differences within Canada in the management of cerebral aneurysms. Methods: A 35 item questionnaire completed by 23 neurosurgeons and neuro-radiologists from Canada. We used descriptive analyses to determine future trends in cerebral aneurysm management as well as the educational implications on the future. Results: Canadians believe that graduating residents are presently 
competent to perform basic procedures like evacuation of a hematoma and clipping a simple $7 \mathrm{~mm}$ middle cerebral artery aneurysm. Most of those surveyed believe that endovascular and open surgical management of aneurysms should be a part of residency training for all residents. Graphs, tables and percentages will be demonstrated. Conclusions: Despite the definite trend towards endovascular management of patients with aneurysms, residency education has not yet been modified to address the needs of patients. Those responsible for the education of future clinicians who will care for patients with cerebrovascular problems should adjust educational objectives and implement learning processes and neurosurgical curricula that will accommodate changing trends in the care of such patients.

\section{P.014}

Psychosurgery revisited: an early medley of neurology, neurosurgery, and psychiatry

\section{A Ganesh (Calgary)* FW Stahnisch (Calgary)}

Background: The controversial era of 20th century psychosurgery, particularly preceding the dissemination of "icepick" lobotomy, witnessed an intriguing early collaboration among neurologists, neurosurgeons, and/or psychiatrists, as exemplified by the partnership of neurologist Walter Freeman (1895-1972) and neurosurgeon James Watts (1904-1994) beginning in 1936. A closer examination of historical publications could reveal important interdisciplinary contributions arising from this period. Methods: The databases of Medline, the National Library of Medicine, and the Surgeon-General's Office were searched using the terms "psychosurgery", "lobotomy", and "leucotomy". Of 283 screened results, 49 met the primary criterion of reporting data pertaining to a 20th century psychosurgical procedure. Results: Several insights into functional neuroanatomy arose from outcomes of these procedures, including an improved characterization of the complex roles of the frontal lobes, thalamus, and amygdala. The cognitive and personality changes undergone by patients post-psychosurgery facilitated the advancement of objective neuro-psychiatric testing. These observations also strengthened the notion that frontal lobes were the centres of personal awareness and identity. Discussion: Attempts by early psychosurgeons to methodically excise portions of the brain and examine their impact on behavior, emotion, and cognition helped establish the organic basis of psychiatric disease. The early inter-disciplinary collaboration that emerged during this era resulted in notable neuroscientific insights that are reflected in modern procedures like stereotactic lesioning/excision and deepbrain stimulation for various neuro-psychiatric conditions.

\section{MOVEMENT DisORDERS (Basic Science, Neurology, Imaging \& Functional Neurosurgery)}

\section{P.015}

Longitudinal quantitative MRI in multiple system atrophy and progressive supranuclear palsy

W Reginold (Toronto)* A Lang (Toronto) C Marras (Toronto) M Alharbi (Toronto) DJ Mikulis (Toronto)

Objective: MRI has been used in parkinsonism to assess atrophy, tissue water diffusivity, and mineral deposition but usually at a single time-point. However, multiple system atrophy (MSA) and progressive supranuclear palsy (PSP) are progressive. This study assessed the value of longitudinal MRI in characterizing the time course of the degenerative process. Methods: Two serial MRIs (mean 22 months apart) were retrospectively analysed blind to diagnosis and scan date in 13 MSA, 6 PSP, and 19 age and sex matched controls. Assessment included apparent diffusion coefficient (ADC) and gradient echo (GRE) intensity ratios from lateral ventricles, caudate, putamen, middle cerebellar peduncle, pons and midbrain. Results: On follow-up imaging, there was a larger ADC increase in the putamen in PSP over time compared to controls $(p=0.047)$. In MSA there was greater volume loss in the pons over time compared to controls $(\mathrm{p}=0.0021)$. Conclusions: Evidence of progressive neurodegeneration can be observed on MRI in MSA and PSP within two years consisting of increasing putaminal $\mathrm{ADC}$ in PSP and pontine atrophy in MSA.

\section{P.018}

An evolving ataxia syndrome - autosomal dominant cerebellar ataxia with deafness and narcolepsy (ADCA-DN) caused by DNMT1 mutations

$J$ Warman Chardon (Ottawa)* A Smith (Ottawa) L Huang (Ottawa) F Farooq (Ottawa) P Bourque (Ottawa) J Woulfe (Ottawa) J Veinot (Ottawa) A MacKenzie (Ottawa) D Bulman (Ottawa) K Boycott (Ottawa)

Background: Autosomal Dominant Cerebellar Ataxia with deafness and narcolepsy (ADCA-DN) is an adult-onset, neurodegenerative disorder causing ataxia, sensorineural deafness and narcolepsy and variably neuropathy, optic atrophy, dementia and seizures. ADCA-DN appears to be caused by mutations in exon 21 of the DNA methyltransferase 1 (DNMT1) gene. Methods: We investigated the clinical, genetic, imaging, electrophysiologic and neuropathologic features from a large multigenerational family with ADCA-DN. Results: The DNMT1 mutation was found in clinically affected patients with a heterozygous DNMT1 c.1709C $>$ T [p.Ala570Val] mutation confirmed by Sanger sequencing. Deafness and ataxia were the first symptoms to appear in the fourth and fifth decade, followed by narcolepsy and cognitive decline. MRI brain imaging revealed cerebral and cerebellar atrophy, with maxillary mucosal thickening in all subjects. Multiple sleep latency tests indicated significant pressure for both non-REM and REM sleep. Nerve conduction studies demonstrated axonal neuropathy. Neuropathological results demonstrated loss of purkinje cells in the cerebellum and relative decrease in hypothalamic orexin 
immunostaining with reactive astrocytes staining for GFAP, consistent with a clinical diagnosis of narcolepsy. DNMT1 is essential for maintenance of methylation, chromatin stability, and gene regulation. Conclusion: This report expands the phenotypical variability of the emerging syndrome adult-onset ADCA-DN caused by DNMT1 mutations.

\section{P.019}

Overexpressing fragments of CREB-binding protein (CBP) to block transcriptional dysregulation and toxicity in a cell model of Huntington's disease

\section{GW Hosier (Halifax)* EM Denovan-Wright (Halifax)}

Background: Huntington's disease (HD) is caused by expression of the huntingtin gene containing an expanded CAG repeat. Nterminal mutant huntingtin protein $(\mathrm{N}-\mathrm{mHtt})$ accumulates in the nucleus and impairs transcription of a subset of genes by binding nuclear proteins. CREB-binding protein (CBP) is a co-activator and acetyltransferase (AT) that binds $\mathrm{N}$-mHtt. We hypothesized that overexpressing $\mathrm{CBP}$ or $\mathrm{CBP}$ fragments would block abnormal interactions of $\mathrm{N}-\mathrm{mHtt}$ in the nucleus and delay $\mathrm{HD}$ progression. Methods: We tested the effect of overexpressing full-length CBP or CBP fragments on transcription (using luciferase promoter assays) and cytotoxicity (using Ethidium Homodimer-1 and Fluoro-Jade C staining) in striatal progenitor cells derived from mice made to express the human huntingtin gene with 7 or 111 CAG repeats. Results: Overexpressing fragments of CBP increased cytomegalovirus and PGC-1 $\alpha$ lpha promoter activity in wild-type and heterozygous HD cells, but not homozygous HD cells. Overexpressing full-length CBP and a CBP fragment containing the AT domain increased toxicity in wild-type cells, while overexpressing a CBP fragment lacking the AT domain had no effect. Overexpressing full-length CBP or CBP fragments decreased toxicity in heterozygous and homozygous HD cells. Conclusions: Overexpressing full-length $\mathrm{CBP}$ or $\mathrm{CBP}$ fragments increased activity of multiple promoters regardless of the presence of $\mathrm{N}-\mathrm{mHtt}$. Excess CBP AT activity was detrimental in wild-type cells, while overexpressing CBP or CBP fragments was protective in HD cells.

\section{P.020}

A case of myoclonus dystonia diagnosed as Tourette's syndrome GW Hosier (Halifax)*

Background: Myoclonus dystonia (M-D) is a rare movement disorder characterized by a combination of myoclonic jerks and mild dystonia typically beginning before age 20. M-D is caused by mutations in the SGCE gene in about $40 \%$ of cases. We report a patient with genetically confirmed M-D who was diagnosed with Tourette's syndrome for 15 years. A similar case was recently reported (Blackburn and Cirillo, Neurology, 2012), indicating the need to consider M-D in patients with early onset myoclonus and dystonia. Methods: This patient was seen in the Movement Disorder Clinic of the QEII Hospital, Halifax, Nova Scotia. Results: Features that indicated M-D were: 1) Early onset ( $<20$ years), 2) myoclonus predominating in the upper body, either isolated or associated with dystonia, 3) positive family history for M-D, 4) exclusion of additional neurological features including cerebellar ataxia, spasticity, and dementia, 5) alcohol-responsive myoclonus, and 6) psychiatric symptoms (obsessive-compulsive disorder, depression, and anxiety). Myoclonic jerks are distinguished from ticks seen with Tourette's syndrome in that they are neither suppressible or proceeded by an urge. Conclusion: Although M-D is rare, it must be considered in patients with early onset myoclonus and dystonia, especially in cases with a positive family history.

\section{P.021}

A clinicopathological study of corticobasal degeneration (CBD) LW Ferguson (Saskatoon)* A Rajput (Saskatoon)

Background: Corticobasal degeneration is a rare form of parkinsonism. There are few autopsy reported series. Methods: Patients seen at Movement Disorder Clinic Saskatchewan (MDCS) with post-mortem pathological diagnosis of CBD were included. Retrospective chart review identified associated clinical features. Results: Seven CBD cases were identified. Mean age of onset (65 years), mean survival (6 years), and mean disease duration prior to first visit (2.6 years) were similar to other clinicopathological studies. The most common initial feature at disease onset was unilateral asymmetric motor dysfunction (5/7), with one case each having gait disorder and cognitive dysfunction, respectively. Six cases used levodopa; one had transient benefit documented while another case had subjective benefit reported by the family but not noted on the neurological exam. While all cases had moderate to prominent nigral neuronal degeneration and a moderate number of typical CBD tau inclusions, the severity of cortical neuronal degeneration and frequency of typical CBD tau inclusions in the cortex varied and did not necessarily correlate with the severity of cortical dysfunction noted clinically. Conclusions: Our findings in general agree with previous clinicopathological reports. We found that the severity of cortical degeneration may not reflect the degree of cortical dysfunction.

\section{NEURO-ONCOLOGY (MEDICAL AND RADIATION ONCOLOGY, IMAGING, Tumour Surgery, Basic Science)}

\section{P.023}

MRI vs. optical coherence tomography: what is the best mode of following patients with compressive pituitary macroadenomas?

WA Ryu (Calgary)* F Costello (Calgary) J Burton (Calgary) $S$ Bhayana (Calgary) B Corenblum (Calgary) L Rudmik (Calgary) B Mechor (Calgary) $Q$ Al Hinai (Calgary) $J$ Williamson (Calgary) $Y$ Starreveld (Calgary)

Background: Estimated prevalence of pituitary macroadenoma is $40 / 100,000$. Patients without visual dysfunction despite contact of the tumor with anterior visual pathways must be followed. A number of different types of MRI analyses have been previously performed including chiasmal elevation, but none has taken into account the large anatomic variation in optic nerve diameter. Growing evidence suggest prognosticative utility of retinal nerve fiber layer thickness (RNFLT) for visual function in patients with compressive tumors. The purpose of this study was to determine whether the severity of anterior pathway compression correlated with intraocular structural changes as measured by RNFLT. Methods: 18 patients (11 females, 
mean age 52 years) with pituitary macroadenomas underwent optical coherence tomography (OCT) testing. MRI analysis was performed by computing a ratio of the cross sectional area of the point of maximum compression to the intraorbital optic nerve. Correlation between degree of compression and RNFLT was sought in 36 optic nerves. Results: Mean RNFLT was $81 \pm 12 \mu \mathrm{m}$ and mean optic nerve compression was $53 \pm 16 \%$. We found no relationship between the RNFLT and degree of optic nerve compression ( $p>0.05)$. Conclusions: Visual function in the setting of anterior visual pathway compression is often undisturbed. This suggests that the traditional longitudinal assessment using high resolution MRI may be a poor predictor of visual function. These findings have important implications for how these patients should be followed.

\section{P.024}

\section{Familial brain tumour in two siblings}

FB Maroun (St. John's)* R Avery (St. John's) T Stuckless (St. John's) L Bowes (St. John's)

Background: Familial gliomas, though reported, are a rare occurrence. We present the case of two siblings with supratentorial malignant gliomas. Method: Case 1: A 9-year-old girl presented with focal seizures of recent onset. Subsequent investigations followed by surgery revealed a left parietal supratentorial PNET. In spite of a wide variety of treatment she died one year after her diagnosis. Case 2: A 14-year-old boy presented with a short history of headache and diplopia. A large left frontal giant cell glioblastoma was removed. He received a course of radiation and chemotherapy and is still alive a year after his surgery with local recurrence. Discussion: Familial syndromes associated with CNS brain tumours are well known. The present cases and other reported series are unique in that there appears to be an obvious genetic association in the absence of any known syndrome. These should be investigated more genetically.

\section{P.025}

\section{Religion and brain tumour patients: a qualitative study}

\section{N Ravishankar (Mississauga)*}

Background: As the focus on modern neurosurgery has shifted to the realm of technological advancement, some patients and their loved ones still hold a strong faith in their religion to guide them through the process. This study aimed to determine whether religion as a coping mechanism was beneficial for patients before, during and after craniotomy. Method: Qualitative case study methodology was used. Semi-structured interviews were conducted with 36 adult patients who underwent surgery for a benign or malignant brain tumour. Interviews were audio recorded and transcribed, and the data subjected to thematic analysis. Results: Four overarching themes emerged from the data: 1) neurosurgical patients said to have significantly benefitted from religion; 2) patients did not require a dedicated religious room in the hospital; 3) patients required religious resources such as leaders and/or groups; and 4) patients were not in favour of their physician engaging in the religious ritual. Conclusions: Most patients found religion to be an effective coping mechanism, offering them strength, comfort and hope through the surgery. The findings from this study emphasise the need for including a "religious time-out" before and after surgery and the inclusion of religious leaders/groups for those in favour to ensure quality care and patient satisfaction.
P.026

Bone marrow derived progenitor cell contribution to tumor neovascularization

\section{K Burrell (Toronto)* G Zadeh (Toronto)}

Introduction: There is emerging evidence that bone marrow derived cells (BMDC) contribute to tumor neo-vascularization and provide a mechanism for cancer cells to evade therapy and reoccur despite maximal therapy and targeted treatments. Our aim is to better understand how anti-angiogenic therapy alters the contribution of Bone Marrow Derived Cells (BMDCs) to malignant brain tumors. Methodology: Using GFP+ bone marrow chimeric mice to engraft intracranial glioma xenografts we will perform realtime in-vivo longitudinal imaging of tumor vasculature and BMDC. We will analyze the mechanism of BMDC contribution to tumor vasculature in response to anti-angiogenic therapy using VEGF-trap alone or used as combinatorial therapy with radiation therapy (RT). Results: BMDCs are recruited to malignant brain tumor in a tumorregional dependent manner. Treatment with VEGFtrap and RT results in loss of regional dependent integration of BMDCs into malignant brain tumor vasculature. Conclusion: Anti-angiogenic therapy disrupts the regional dependent contribution of BMDC to tumor neovascularization potentially providing a mechanism by which tumors can evade therapy. Clinically being able to disrupt the regional dependent contribution of BMDCs to tumor neovascularization and hence target the influx of BMDCs following treatment would provide a novel and promising therapeutic strategy to prevent reoccurrence through re-vascularization mechanisms.

\section{P.027}

Management strategies of diffuse low-grade gliomas in Canada - a multi-center questionnaire

OH Khan (Toronto)* P Kongkham (Toronto) WP Mason (Toronto) G Zadeh (Toronto)

The management of adult diffuse low-grade gliomas (LGG) is variable. The level of evidence to support clinical care remains controversial and we are faced with the daily challenge of designing the best management strategy for individual patients. Controversies are, in general, about a "wait-and-see" strategy, diagnostic workup, surgical intervention, postoperative imaging, adjuvant treatment, and follow-up. In order to better understand the practice trends across Canada we will present a questionnaire that surveyed neurosurgeons, radiation oncologists, and medical oncologists (neuro-oncology), neuropathologists and neuroradiologists involved in treating low-grade gliomas. We will present the results of our survey in order to demonstrate the range of existing clinical practice across Canada, identify specific Canadian health care relevant and applicable practice patterns and reflect areas in need of further development. Furthermore, our hope is that the survey results will provide a forum to engage clinicians involved and interested in treating low-grade gliomas to form a nation working-group that can focus on identifying clinical needs of medical communities involved in the care of these patients. 


\section{P.028}

Institutional review and identification of indications for endoscopic expanded endonasal approach to olfactory groove and tuberculum sellae meningiomas

OH Khan (Toronto)* G Klironomos (Toronto) D Holliman (Toronto) A Vescan (Toronto) F Gentili (Toronto) G Zadeh (Toronto)

Several microsurgical transcranial approaches have been utilized for resection of anterior cranial fossa meningiomas. However, debate continues as to whether these approaches may be more prone to injury of the optic apparatus or it's blood supply. The definitive indications for using the endoscopic expanded endonasal (EEE) approach are not yet clear. We report our experience of the EEE approach for tuberculum sellae and olfactory groove meningiomas using the endoscope as the sole means of visualization (i.e. "pure" endoscopic). In addition, we reviewed and pooled the available literature regarding the use of the pure EEE approach for these lesions. Following local research ethics board approval a prospectively maintained database of all surgical cases between May 2006 and August 2012 was retrospectively reviewed. A PubMed literature search was performed and articles were retrieved, reviewed for patients undergoing pure EEE approaches in addition to all references therein being assessed for further relevant cases. Single case reports were excluded. At the time of abstract submission the results of our series are being analyzed. Results of literature search will be presented in table format. Pure EEE approach for anterior cranial fossa meningiomas can be safely utilised by surgeons appropriately experienced in endoscopic endonasal procedures.

\section{P.029}

Role of microRNA-mediated mechanisms for maintenance of glioma derived stem cells properties

SK Singh (Toronto)* S Agnihotri (Toronto) A Vartanian (Toronto) S Jalali (Toronto) K Burrell (Toronto) J Gumin (Houston) EP Sulman (Houston) FF Lang (Houston) G Zadeh (Toronto)

Role of cancer stem cells in tumor formation and tumor heterogeneity is currently one of the most researched topics in cancer biology. A better understanding of molecular mechanisms regulating the biology of cancer stem cells may ultimately help provide a better management of cancer patients. Various individual or families of microRNAs have been shown to have oncogenic or tumor suppressor function in high-grade gliomas. MiRNAs have functional relevance in regulation of critical genes and parameters implicated in glioma stem cell (GSC) behavior and differentiation. We have investigated global role of microRNA in GSCs after biogenesis of microRNA is compromised. Analysis of TCGA database suggests that high Dicer expression level is correlated with better prognosis of glioblastoma (GBM) patients. Gene signatures correlated with Dicer expression levels suggest Dicer/miRNA mediated mechanisms potentially regulate a multitude of cellular pathways in GBMs. Immunohistochemistry analysis of GBM tissue microarray reveals that approximately $48 \%$ of GBM patients have low or undetectable levels of DICER1 protein. We have utilized various in vitro approaches (including exposure to hypoxia) to characterize the GSC properties after knockdown of Dicer. Furthermore, we have applied biochemical methods to establish direct miRNA-mRNA interaction network relevant to GSCs. The scope and importance of these results from GSCs will be discussed specifically in context of GBM and GBM heterogeneity.

\section{P.031}

\section{Role of MMP16 in malignant and invasive meningioma} S Jalali (Toronto)*

Skull base meningiomas are typically managed by surgical resection. However in the instances of malignant, hyperostotic or bone invasive skull base meningiomas complete surgical resection becomes challenging. We aimed to identify novel signaling molecules mediating brain and bone invasion of meningiomas in order to be able to identify targeted therapeutics. Using micro-array analysis on malignant versus non-malignant, plus bone invasive and non-invasive meningiomas, we identified over 200 differentially expressed genes. For the purposes of this study we focused on the role of MMP16 in bone invasive and malignant meningiomas. We confirmed elevated expression of MMP16 in malignant and bone invasive meningiomas using real-time PCR analysis. To study the in vitro and in vivo characteristic of meningioma cell lines, we transfected cells to over-express and under-express MMP16. Knockdown of MMP16 showed statistically significant reduced invasion in vitro. Furthermore, knock-down of MMP16, using zymography, we demonstrated an alteration in activity of other MMPs including MMP2 and MMP9. Knock-down of MMP16 reduced tumor progression, as confirmed by volumetric assessment of serial MRI images of xenograft meningioma tumors. In summary, our data supports a role for MMP16 in invasive phenotype of the meningioma tumors and supports targeting MMP-16 as a potential therapeutic target in aggressive meningiomas. Ongoing studies are focused on pre-clinical and potentially clinical studies to establish the clinical value of targeting MMP16.

\section{P.032}

Analysis of growth rate and growth patterns in pituitary adenomas: correlations to MIB-1, p27, and FGFR4

\section{E Monsalves (Toronto)*}

Objective: To establish whether the growth and invasion pattern of pituitary adenomas (PA) correlate with tumor proliferation and growth factors expression. Materials: PA operated on at our institution between 1999-2011 were reviewed for this study. We calculated pre- and post-operative tumor volume doubling time (TVDT). We recorded patient demographics, PA growth patterns and compared to the expression profile of the MIB-1 LI, p27, and FGFR4. Results: $67 \%$ were clinically non-functioning PA and $33 \%$ were functioning. There were $53 \%$ women and $47 \%$ men with an average age of 53. The preoperative TVDT was positively correlated with patient age in all PA. Preoperative TVDT was inversely correlated with the MIB-LI. Gonadotrophs were associated with a faster TVDT if there was negative p27 and positive FGFR4 expression. Null cell and somatotroph adenomas had a lower mean TVDT when there was a cystic/hemmorhagic component. Somatotrophs were also associated with a faster TVDT if the tumor had optic chiasm compression. There were $34.6 \%$ postoperative residuals, $41.5 \%$ of which re-grew. The presence of residual tumor was related to several recorded preoperative growth patterns. Postperative TVDT exhibited a significant correlation to preoperative TVDT. Conclusions: The preoperative TVDT correlates with 
postoperative TVDT, which is useful for treatment planning. Furthermore, the MIB-1, p27, and FGFR4 are associated with TVDT in PA providing evidence for the clinical relevance of these biomarkers.

\section{P.033}

\section{A case of a lung adenocarcinoma metastasizing to scalp}

F Salehi (London)* BH Wang (London) J Lau (London) J Megyesi (London)

Background: Lung adenomacarcinoma metastasis to scalp and skull has not been previously reported. Methods: A 61-year-old female presented with a scalp mass that increased in size from one $\mathrm{cm}$ to $10 \mathrm{~cm}$, over a 7-month period. She had a recent history of $201 \mathrm{~b}$ weight loss. Results: CT scan revealed a soft tissue mass in left frontal scalp involving the underlying bone and thickening of dura. Magnetic Resonance Imaging (MRI) three months later exhibited rapid growth of the lytic lesion. Bone scan showed no other primary lesions. Intraoperative biopsy specimen displayed histological characteristics of an adenocarcinoma. The patient was pan-scanned and a primary lung lesion with extensive hilar lymphadenopathy was identified. She subsequently underwent operative resection of the lesion and cranioplasty. Pathological examination of tumor biopsy showed a moderately differentiated adenocarcinoma characterized by large irregularly shaped acini embedded in a desmoplastic stroma with a mixed acute and chronic inflammatory infiltrate. Mitotic figures were encountered. The neoplastic cells were immunopositive for CK-CAM5.2, CK 7 and TIF-1 (nuclear), and immunonegative for CK 20, features in keeping with adenocarcinoma. Conclusions: We describe a rare case of metastasis from a lung adenocarcinoma to skull. The histopathological features and differential diagnosis of such lesions are discussed.

\section{NeUROMUSCULAR (BASIC SCIENCE, EMG/NCS \& Peripheral Nerve Surgery)}

\section{P.034}

\section{Secondary hypokalemic periodic paralysis}

\section{A Jahangirvand (Saskatoon)* EJ Atkins (Saskatoon) G Hunter (Saskatoon)}

Background: We report a case of hypokalemic periodic paralysis (HypoPP), likely secondary to gastrointestinal losses. Method: Case report. Results: A 31-year-old First Nations male presented to hospital after developing generalized weakness of his trunk and extremities. The weakness was noted immediately on waking after normal function the night prior. He had been overindulging on carbohydrates and had experienced significant diarrhea the previous day. His vital signs were stable and neurologic exam revealed $0 / 5$ strength in the lower limbs and 2/5 strength in the upper limbs. The rest of the examination, including deep tendon reflexes, was normal. Laboratory analysis showed a potassium of $1.8 \mathrm{mEq} / \mathrm{L}(3.5-5$ $\mathrm{mEq} / \mathrm{L}$ ) and normal thyroid studies. After replacement of potassium his symptoms resolved within hours. Genetic testing for CACNA1S and SCN4A did not identify any mutations. Discussion: HypoPP can be the result of several underlying conditions requiring systematic evaluation. The clinical manifestation is similar to familial HypoPP, where a mutation in CACNA1S or SCN4A is found in approximately $80 \%$ of patients. A profoundly low potassium level (less than $2 \mathrm{mEq} / \mathrm{L}$ ) suggests (but does not prove) a secondary cause of periodic paralysis. It is important for clinicians to investigate for gastrointestinal potassium losses, since this unusual presentation of severe weakness usually rapidly resolves with normalization of serum potassium.

\section{P.035}

Effectiveness of second corticosteroid injections for carpal tunnel syndrome

\section{NL Ashworth (Edmonton)* J Bland (Canterbury)}

Background: A single local corticosteroid injection is an effective treatment for carpal tunnel syndrome. No existing studies specifically examine the effectiveness of a second injection on relapse after primary injection. Methods: We identified a cohort of patients who had received an initial corticosteroid injection into one wrist and then, at a later date, a second injection into the same wrist. We then compared the change in the Boston symptom severity scale (SSS) and functional status scale (FSS), and relapse time between the first and second injections for each patient. Results: In 229 patients who received two injections the mean improvement in SSS was 1.2 (sd 0.8) for the first injection and 1.3 (sd 0.9) for the second, not statistically significant. Mean improvement in FSS for the first injection was $0.4(\mathrm{sd} 0.8)$ and 0.7 (sd 0.8 ) for the second, which was statistically significant $(\mathrm{p}<0.001)$. The mean time to relapse for the first injection was 336 (sd 180) days and for the second injection 348 (sd 182) days, which was not statistically significant. Conclusions: Second corticosteroid injections appear to be at least as effective and long lasting as the first injection.

\section{P.037}

Paraneoplastic stiff person syndrome mimicking tetanus: a case series report and literature review

J Madhani (Vancouver)* K Chapman (Vancouver) S Cresswell (Vancouver) G Gibson (Vancouver)

Background: Stiff Person Syndrome (SPS) results from an imbalance between excitatory aminergic and inhibitory GABAergic and glycinergic systems, and can be paraneoplastic. Methods: We describe a case series of two patients presenting with trismus, who were initially treated for tetanus. Both subsequently developed long tract findings, neuropathic pain, lower extremity stiffness and myoclonus. One patient had known bladder cancer at the time of presentation, and the second was subsequently diagnosed with small cell lung cancer. Serologic testing revealed antibodies against glycine receptors in both. Other paraneoplastic antibodies were negative. Both responded to immunomodulatory treatment (IVIg and rituximab), in combination with treatment of their underlying cancers. Results: Literature review found three similar cases of Stiff Person Plus Syndrome with Progressive Encephalomyelitis with Rigidity and Myoclonus (PREM) with associated positive antibodies against glycine receptors. Glycinergic cells are important inhibitory cells at the spinal level. Glycinergic cells are also a target of the 
tetanus toxin. Conclusions: Anti Glycine may be an important antibody associated with Stiff Person Plus variant of Progressive Encephalomyelitis with Rigidity and Myoclonus (PREM). Paraneoplastic SPS should be considered in patients presenting with trismus.

\section{STROKE (Vascular Neurology, Imaging, Basic Science, Neurovascular/ ENDOVASCUlar SURGERY)}

\section{P.038}

\section{Rock 'til you drop: cervical artery dissection in musicians}

KE Beadon (Vancouver)* D Johnston (Vancouver) K Chapman (Vancouver) PA Gooderham (Vancouver) P Teal (Vancouver)

Background: Cervical artery dissection (CAD) is a rare but potentially devastating cause of stroke. CAD may be secondary to trauma, trivial trauma, or idiopathic. Repetitive neck flexion/ extension to the beat of the music is an unusual cause of trauma to the neck vessels. Most patients present with neck pain; TIA or stroke may occur at the time of presentation or in the days that follow. Methods/Case Descriptions: Two rock musicians presented with CAD. A 30-year-old male base guitar player, presented with headache and increasing unsteadiness. CT angiogram demonstrated a vertebral artery dissection and a significant posterior fossa infarct requiring urgent surgical decompression. A 40-year-old male guitar player presented with headache and transient right hemiparesis after a concert and had a carotid artery dissection. No mechanism other than guitar playing was identified. Results/Discussion: The majority of CAD is thought to be idiopathic however, neck flexion/extension is a risk factor. Neither of the described patients had any known risk factors for CAD. Rigorous guitar playing with "head banging" may have contributed to the dissections seen. Both were treated with ASA and one patient had significant infarct and lasting neurologic symptoms; the other patient had no deficits. Conclusion: Two cases of CAD in healthy young musicians are described. Rhythmic neck flexion/extension while playing rock music may be a risk factor for CAD.

\section{P.040}

Delayed migration of the pipeline embolization device stent following treatment of an internal carotid artery aneurysm: the need for early follow-up imaging

\section{Z Tymchak (Saskatoon)* D Fiorella (Stony Brook) M Kelly (Saskatoon)}

Background: The Pipeline embolization device (PED) has become an alternative for treatment of broad-necked aneurysms. Stent migration can lead to residual aneurysm filling. We report the second case of delayed proximal stent migration. Methods: A 60year-old female with a three month history of dizziness with occasional nausea and vomiting. She was found to have a $10.4 \mathrm{~mm}$ broad-necked left internal carotid artery (ICA) aneurysm on computed tomography angiography (CTA). She underwent treatment for this aneurysm with a PED. Two PED stents $(4.75 \times 14$ $\mathrm{mm}$ and $4.75 \times 18 \mathrm{~mm}$ ) were deployed with good intra-operative result. Results: six week follow-up imaging showed proximal migration of the stent with residual aneurysm contrast filling. The stent was oriented such that the contrast jet was directed into the dome of the aneurysm. The patient was also complaining of increasing headaches. She therefore underwent a second treatment with a single $4.75 \times 20 \mathrm{~mm}$ PED stent to cover the neck of the aneurysm. Follow-up imaging at four months revealed obliteration of the aneurysm. Conclusions: Proximal migration of the PED can occur in a delayed fashion leading to residual aneurysm filling. The time course for delayed stent migration is unclear. We recommend early follow-up imaging after PED placement to ensure timely definitive management of this problem.

\section{P.041}

Incidence of hemorrhage in a cohort of patients with unruptured intracranial aneurysms

S Ahmed (Saskatoon)* E Barber (Saskatoon) ME Kelly (Saskatoon)

Background: Natural rate of aneurysm rupture remains controversial, especially for small aneurysms. Centers frequently choose to follow rather than treat aneurysms $<7 \mathrm{~mm}$. We aim to study the safety of observation in patients with small UIAs followed in Saskatchewan. Methods: We conducted a retrospective review of all patients in a prospective database presenting between July 2008 and June 2012 with unruptured aneurysms. Aneurysm characteristics recorded included size, presentation, and follow-up imaging. Multiple aneurysms in a single patient were considered independently. Patients with no follow-up were excluded from the analysis, including those still awaiting initial follow-up. Results: Of the 203 UIAs $<7 \mathrm{~mm}, 25$ were treated, while mean follow-up time was 12.3 months for followed aneurysms, with two incidences of rupture. Of the 88 UIAs $\geq 7 \mathrm{~mm}, 42(48 \%)$ were treated. Mean follow-up time for followed aneurysms was 8.2 months, and there was one incidence of rupture during follow-up. Conclusions: Treatment decision paradigms used in our center showed low rates of rupture in untreated aneurysms less than $<7 \mathrm{~mm}$.

\section{P.042}

Minimally invasive subcortical parafascicular access clot evacuation (MiSPACE)

BR Ritsma (Ottawa)* A Kassam (Ottawa) D Dowlatshahi (Ottawa) T Nguyen (Ottawa) G Stotts (Ottawa)*

Background: Spontaneous intracranial hemorrhage (ICH) is common and causes significant mortality and morbidity. To date, optimal medical and surgical intervention remains uncertain. A lack of definitive benefit for operative management may be attributable to adverse surgical effect, collateral tissue injury. This is particularly relevant for ICH in dominant, eloquent cortex. Minimally invasive surgery (MIS) offers the potential advantage of reduced collateral damage. MIS utilizing a parafascicular approach has demonstrated such benefit for intracranial tumor resection. Methods: We present a case of dominant hemisphere spontaneous ICH evacuated via the minimally invasive subcortical parafascicular access clot evacuation (MiSPACE) model. We use this report to review the literature on ICH surgical management, introduce MiSPACE, and to examine the application of this novel MIS paradigm. Results: The featured patient presented with a left temporal ICH and severe global aphasia. 
The hematoma was evacuated via the MiSPACE approach. Postoperative reassessments showed significant improvement. At two months bedside language testing was normal. MRI tractography confirmed limited collateral injury. Conclusions: This case illustrates successful application of the MiSPACE model to ICH in dominant, eloquent cortex. MRI tractography illustrates collateral tissue preservation. Safety and feasibility studies are required to further assess this promising new therapeutic paradigm.

\section{P.043}

Clinician's guide to outcome prediction in clinical neurosciences: application of bayesian neural networks with fuzzy logic inferences

$B W$ Lo (Toronto)* R Macdonald (Toronto) A Baker (Toronto) M Levine (Hamilton)

Background: Complex relationships exist among heterogeneous groups of prognostic factors. In real life, data points rarely fit perfectly linear relationships. Greater deviations from linearity point to the need for exploratory analyses with complex non-linear systems. Methods: The novel approach of Bayesian Neural Networks with Fuzzy Logic Inferences was applied with data from 4 trials of tirilazad for aneurysmal subarachnoid hemorrhage that included 3561 patients. Validation was carried out to increase generalizability of this approach. Results: Neural Networks with Bayesian Regularization of 30 prognostic variables revealed 11 latent variables in one layer using MATLAB. Internal validation was performed with discrimination c-statistic (ROC curve) of 0.84 . Fuzzy logic inference rules were then created for external (domain) validation, and impact analysis in ambispective patient cohorts. Conclusions: Conceptual generalization of Bayesian Neural Networks with Fuzzy Logic Inferences is as follows: Based on one's own experience (summation of existing parameters weighted by strength of belief in what happened beforehand), one can predict (based on one's assigned strength of belief) where along a spectrum of probabilities the unknown quantity value will end up. If it falls outside the spectrum in real life, then, one has to check whether there are still unknown elements influencing the outcome variable in question.

\section{P.044}

Health related quality of life following aneurysmal subarachnoid hemorrhage - a qualitative synthesis of determinants and measurement

BW Lo (Toronto)* B Ja Ja (Toronto) V McCredie (Toronto) J Lim (Dublin) J Ramkumar (Sudbury) R Takroni (Riyadh) N Khezri (Vancouver) A Louffat-Olivares (Toronto) $J$ Spears (Toronto) $R$ Macdonald (Toronto)

Background: More than half of aSAH patients who recover to functional independence experience chronic reductions in health related quality of life (HRQoL). Longitudinal studes show residual physical disability as key predictor of decreased HRQoL. Many of these studies face methodological challenges, including those of reporting and measurement. Methods: Systematic review of current literature on aSAH HRQoL was performed, with emphasis on qualitative thematic saturation. Results: Under self-reported and diagnosed psychological determinants of decreased HRQoL include cognitive deficits (executive functioning, visuospatial function, attention, verbal memory, information processing and reaction time), mood and emotional complaints (depressive symptoms, anxiety, post-traumatic stress, loss of motivation, abnormal introversion, lability), personality and vitality changes (passive coping, fatigue and vitality deterioration with decreased energy and tolerance to mild stressors). Methodological issues involve those of reporting: patient selection bias and observation bias. Measurement issues include capturing discriminative health measurement scales with requirements of validity, reliability and responsiveness. Conclusions: Methodological limitations may be overcome with longitudinal mixed methods studies of HRQoL with both qualitative aspect (phenomenological or thematic exploration of HRQoL domains) and quantitative aspect with use of stroke measurement scales to capture multidimensional HRQoL domains. Random effects models should be specified a priori to acknowledge account for both within and between study variability.

\section{P.045}

Cerebellar stroke presenting with global aphasia in a patient with muscular dystrophy

A Jahangirvand (Saskatoon)* E Haghir (Saskatoon) G Hunter (Saskatoon)

Background: We report a case of global aphasia resulting from right cerebellar ischemic stroke, on a background of limb-girdle muscular dystrophy. Method: Case report Results: A 49-year-old right-handed Caucasian male, with a long-standing history of presumed limb-girdle muscular dystrophy, presented to hospital with acute onset of speech impairment. Neurologic examination was significant for mutism, impaired naming, and reduced comprehension and repetition. Reading and writing were similarly impaired. There was no nystagmus. Testing for strength and coordination was limited due to his muscle disorder, but there were no apparent new deficits. MRI of the brain revealed a focus of diffusion restriction involving the right cerebellar hemisphere. Extensive testing did not reveal a source, including detailed cardiac investigations. His language impairment gradually resolved and he was close to his baseline within the next few days. At three month follow-up his language was entirely normal, and there were no cerebellar deficits apparent. Discussion: An important connection between the cerebellar hemisphere and the contralateral cerebrum exists. Investigators have suggested that the right cerebellar hemisphere appears to be associated with language and the left cerebellar hemisphere with visuospatial functions. This case adds evidence to this view that language impairment in cerebellar mutism may be a consequence of "crossed cerebello-cerebral diaschisis".

\section{P.046}

\section{Pathophysiology and genetic determinants of subarachnoid hemorrhage}

JA D'Abbondanza (Toronto)* J Ai (Toronto) R Macdonald (Toronto)

Subarachnoid hemorrhage $(\mathrm{SAH})$ can occur traumatically or spontaneously. Although SAH only accounts for approximately $7 \%$ of all strokes worldwide, it is associated with a greater proportion of morbidity and mortality. The goal of this study was to investigate the potential contribution of different genetic backgrounds to the secondary complications after SAH in a mouse model. SAH was induced in 7 inbred strains of mice, and the degree of large artery vasospasm and brain injury was assessed. After 48 hours, SAH mice 
showed a significant reduction in middle cerebral artery diameter compared to sham counterparts $(\mathrm{P}<0.05$, Student's t-test $)$, with the exception of KK/HIJ and DBA/2 mice. Neuronal injury in the cerebral cortex, as determined by fluoro-jade positive cells, was exhibited across all strains compared to sham mice $(\mathrm{P}<0.05$, Student's t-test). A/J and DBA/2 mice differed significantly from $\mathrm{C} 57$ mice in the number of degenerated neurons $(\mathrm{P}<0.05$, ANOVA, Fisher LSD) and demonstrated the two extremes of brain injury. Additionally, no correlation was found between brain injury and vasospasm, which suggests a disconnect between vasospasm and neuronal injury. Further microarray investigations of A/J and C57 mice may also provide invaluable insight into the causes of these inter-strain differences and may more accurately identify which genetic contributors are responsible for secondary complications after SAH.

\section{P.047}

Reversible cerebral vasoconstriction syndrome associated with autonomic dysreflexia: a case report

A Trudelle (Québec)* M Savard (Québec) S Verreault (Québec) M Audet (Québec)

Background: Reversible cerebral vasoconstriction syndrome (RCVS) is characterized by recurrent thunderclap headaches and multifocal segmental vasoconstrictions of the cerebral blood vessels with or without focal neurological deficits. RCVS has already been reported in various clinical settings. It has been reported once before in association with autonomic dysreflexia (AD) in a patient with a traumatic spinal cord injury. Methods: Case report. Results: A 52year-old man with a residual spastic quadriparesis from a traumatic C5 fracture experienced recurrent thunderclap headaches. He also experienced episodes of AD due to manipulation of an indwelling catheter before each thunderclap headache. A CT scan of the head, a brain MRI and a lumbar puncture were normal. A CT angiography of the brain vessels revealed multiple segmental vasoconstrictions of the main cerebral arteries. A follow-up CT angiography of the brain vessels three months later showed normalization of the segmental vasoconstrictions and a diagnosis of RCVS due to AD was made. Conclusions: This is the second reported case of RCVS associated with autonomic dysreflexia thus rendering more credible that clinical association. This association supports the theory of abnormal sympathetic activity, which is observed in $\mathrm{AD}$, as a component of the underlying pathophysiology of RCVS.

\section{P.048}

\section{Tran arterial embolization of spinal pial AVM}

\section{SM Alrashidi (Halifax)* S Christie (Halifax) J Shankar (Halifax)}

We described a 39-year-old woman who presented to emergency department with a post coital headache and neck pain. Her symptoms lasted approximately 20 minutes with her headache resolving over the 2 hours before her presentation to Emergency. Neurological examinations were benign and CT of her head reveled no acute findings. A CT angiogram reveled no dissection and no aneurysm of her intracranial arterial system. A lumber puncture was performed, which reveled elevated protein, red blood cells and white blood cells. An MRI of her cervical spine was performed on post admission day 1 , which reveled what was believed to be consistent with a small vascular malformation in the cervical spinal cord at the level of C2 and C3. Differntial diagnosis were Spinal pial AVM or a cavernoma. The patient underwent a Tran arterial embolization of the above- mentioned spinal Dural AV fistula. The procedure was successful in partially emboli zing the lesion. She remained in the hospital for two weeks and was then discharged.

\section{P.049}

The role of intra-aortic balloon counter-pulsation pumps in subarachnoid hemorrhage patients with refractory symptomatic vasospasm: a successful case report and review of the literature $V$ McCredie (Toronto)* S Ramachandran (Toronto) B Lo (Toronto) RL Macdonald (Toronto) A Baker (Toronto)

Background: Subarachnoid hemorrhage is a devastating disease; up to $15 \%$ die from the initial rupture and approximately one third of those who survive remain dependent with respect to daily activities. Secondary complications include delayed cerebral ischemia (DCI) and myocardial dysfunction. The use of mechanical augmentation, with intra-aortic balloon counter-pulsation pump (IABP) technology, for severe left ventricular failure has been reported in the literature. Methods: We report the case of a 43-yearold patient with a ruptured anterior communicating aneurysm who went on to develop severe vasospasm. This was complicated by severe left ventricular failure in the presence of pharmacological hemodynamic augmentation. An IABP was inserted successfully and the patient made a full recovery. Results: A review of the literature revealed only eight publications in the past 16 years, with a total number of 15 patients reportedly treated with IABP for refractory vasospasm and myocardial dysfunction. Only one of these 15 patients made a full recovery (GOS 5). Our case is the second documented patient to make a full recovery. Conclusions: Contemporary therapies to prevent DCI, including pharmacological hemodynamic augmentation and cerebral balloon angioplasty, may be insufficient or exhausted in a subset of patients. Our case demonstrates the effective use of an IABP as rescue therapy.

\section{P.050}

SAH with multiple intracranial aneurysms: pitfalls in endovascular treatment

BH Wang (London)* B VanAdel (London) E Dyer (London) D Lee (London) D Pelz (London) M Boulton (London) S Lownie (London)

Background: Ruptured intracranial aneurysms are the most common cause of spontaneous subarachnoid hemorrhage (SAH), accounting for $75-80 \%$ of cases. When an aneurysm is identified, it is important to assess for additional aneurysms. The incidence of multiple aneurysms ranges from $20-30 \%$. With ruptured aneurysms, the primary therapeutic goal is to secure the aneurysm by isolating it from the circulation in order to prevent re-rupture. Method: We present four patients with spontaneous SAH and multiple intracranial aneurysms. All were treated initially with endovascular coiling immediately after conventional cerebral angiography. Results: Three out of four patients were subsequently found to have the wrong aneurysm coiled. Two of the patients went on to urgent microsurgical clipping of the remaining aneurysm(s) and were found to have had the wrong aneurysm coiled. A third patient also had the wrong aneurysm coiled and experienced re-rupture, requiring a second coiling procedure to secure the ruptured aneurysm. Additional morbidity was incurred in these cases as a result. 
Conclusion: When multiple aneurysms are identified in SAH, it may be a challenge to correctly identify the ruptured one, which must be treated first. Little is reported in the literature regarding this problem. This report highlights this dilemma, which seems to be increasing along with the increased use of endovascular aneurysm coiling - a technique less likely to confirm rupture site as compared to surgical clipping.

\section{P.051}

\section{Molecular alterations in hippocampus underlying the loss of} LTP after subarachnoid hemorrhage

SM Han (Toronto), H Wan (Toronto) G Kudo (Toronto) WD Foltz (Toronto) DC Vines (Toronto) DE Green (Toronto) $T$ Zoerle (Toronto) A Tariq (Toronto) S Brathwaite (Toronto) J D'Abbondanza (Toronto) J Ai (Toronto) R Macdonald (Toronto)*

Patients who survive aneurysmal subarachnoid hemorrhage (SAH) often have deficits in learning, memory and executive function. We have previously reported that rodents with SAH develop cognitive deficits and loss of long-term potentiation (LTP). Here we investigate the potential mechanisms underlying the loss of LTP. SAH was created by injection of $300 \mu 1$ of fresh unheparinized arterial blood into the prechiasmatic cistern of Sprague-Dawley rats. In the dendritic area of CA1 neurons, the number of synapses was significantly decreased in SAH models compared to controls. Similarly, the expression of GluR1, GluR2 and CaM kinase II was significantly decreased in SAH rats. A decreased level of superoxide but increased level of NO was detected in SAH animals as compared to controls. These results suggest that the loss of LTP after SAH in rat model may be due to a synaptic plasticity rather than cell death. Decreased immunoreactivity to GluR1, GluR2 and CaM kinase II in $\mathrm{SAH}$ animals suggests reduction in key protein mediators necessary for the maintenance of LTP may simultaneously contribute to the loss of LTP. Decreased superoxide and increased nitric oxide (NO) levels in SAH models suggests oxidative stress is involved in the loss of LTP. The identification of potential mechanisms involved would provide novel therapeutic targets and a better understanding the causative factors of cognitive deficit in patients with SAH.

\section{P.052}

Avoid delay in giving IV tPA to achieve timely reperfusion with bridging therapy

S Mishra (Calgary)* M Almekhlafi (Calgary) J Desai (Calgary) V Nambiar (Calgary) M Eesa (calgary) B Menon (Calgary) M Goyal (calgary) A Demchuk (Calgary)

Background: There is suspected advantage of bridging (IV+IA) therapy. We studied whether bridging therapy influenced successful reperfusion compared to the endovascular approach alone. Methods: We analysed anterior circulation ischemic stroke patients treated with stentriever devices, with or without IV tPA, between January 2011 to June 2012. Patient clinical and radiologic data were collected. Outcomes included 24-hour NIHSS improvement and sICH (SITS-MOST definition). Results: Summarized in table. IV tPA resulted in delay of 26 minutes on average, in the time from imaging to endovascular reperfusion compared to the IA only group ( $\mathrm{p} 0.03$ ). However, this delay did not significantly impact the median improvement in 24-hour NIHSS, as these patients were still treated relatively early from onset time (median of 130 minutes). Incidence of sICH was slightly higher in the bridging group, but did not reach significance (p 0.5). Conclusion: Randomized trials should be designed to avoid delays caused by IV tPA administration to achieve timely endovascular reperfusion. Our findings do not identify any added risks associated with the use of bridging approach.

Table 1 (P.052)

\begin{tabular}{|l|c|c|}
\hline & IV+IA & IA alone \\
\hline Number of patients & 64 & 41 \\
\hline Median age (IQR) & $68(19.5)$ & $68(18)$ \\
\hline Median baseline NIHSS & $18(9)$ & $17(7)$ \\
\hline Median baseline ASPECTS & $8(2)$ & $8(3)$ \\
\hline Proportion with cervical ICA occlusion & $10.9 \%$ & $4.9 \%$ \\
\hline Proportion with terminal ICA occlusion & $12.5 \%$ & $7.3 \%$ \\
\hline Proportion with M1 MCA occlusion & $81.3 \%$ & $48.8 \%$ \\
\hline Proportion with M2 MCA occlusion & $15.6 \%$ & $17.1 \%$ \\
\hline Median door to imaging (mins) & $20.5(14)$ & $20.5(11)$ \\
\hline Median imaging to puncture (mins) & $65(32)$ & $44(43) \#$ \\
\hline Median puncture to reperfusion (mins) & $42(44)$ & $39(51)$ \\
\hline Median imaging to reperfusion (mins) & $113(62)$ & $87(62) \#$ \\
\hline Median onset to IV tPA (mins) & $130(111)$ & $\mathrm{NA}$ \\
\hline Median onset to reperfusion (mins) & $250(123)$ & $305(237)$ \\
\hline Proportion with TICI 2b-3 reperfusion & $78.1 \%$ & $80.5 \%$ \\
\hline Proportion with TICI 2c \& 3 reperfusion & $48.4 \%$ & $39 \%$ \#\# \\
\hline Median 24-hour NIHSS & $6(11)$ & $9(10)$ \\
\hline Median 24-hour ASPECTS & $7(3)$ & $6(4)$ \\
\hline Median NIHSS drop in 24 hours & $11(11)$ & $8(6) \dagger$ \\
\hline Proportion with 50\% or more drop in NIHSS in 24 hours & $70.3 \%$ & $53.7 \%{ }^{*}$ \\
\hline Symptomatic ICH in 24-hour CT (SITS-MOST) & $7.8 \%$ & $4.8 \% *^{* *}$ \\
\hline & & $\begin{array}{l}* \\
\text { Chi-square p0.08 }\end{array}$ \\
\hline
\end{tabular}

\section{$\mathbf{P . 0 5 3}$}

Aneurysm coiling- 16 years experience in a single Canadian center

A Bata (Halifax)* W Maloney (Halifax) $R$ Vandorpe (Halifax) $J J$ Shankar (Halifax)

Background: Endovascular coiling of aneurysms has now become a preferred treatment in most of the Canadian centers. We were one of the early adopter of this technique in Canada. The purpose of this study was to evaluate our endovascular coiling experience for intracranial aneurysms. Material and Methods: We retrospectively reviewed our endovascular database in the department of Neuroradiology to assess all interventional cases done in our center. The distribution of intracranial aneurysms, the method and outcome of treatment were recorded. Results: From 1997 to 2012, we had done a total of 1269 endovascular procedures. Out of these 767 were endovascular treatments for intracranial aneurysms. Out of these 197 aneurysms were located in the posterior circulation and others in the anterior circulation. The most common location for the treated aneurysms was anterior communicating artery $(n=220)$ followed by basilar tip $(n=118)$ and posterior communicating artery $(n=117)$. Most of the aneurysms were treated either with conventional coiling or balloon assisted coiling. Stent assisted coiling was used in only 37 patients. SILK flow diverter was used in 23 patients. Conclusion: Our study shows the evolution of endovascular technique in the treatment of intracranial aneurysm over the last 16 years in a medium size Canadian center. 


\section{$\mathbf{P . 0 5 4}$}

Concurrent Solitaire FR use and carotid stenting in acute tandem carotid occlusion setting

IM Alnaami (Edmonton)* R Owen (Edmonton) J Rempel (Abha)

Background: Treatment of tandem acute internal carotid artery (ICA) occlusion and middle cerebral artery (MCA) occlusion represents a challenge from a neurointerventional point of view. Multiple modalities and approaches are described in the literature with limited evidence on each. Method: Three patients presented with acute stroke symptoms and signs. Computed tomography (CT) and $\mathrm{CT}$ angiogram revealed tandem ICA and MCA occlusions. Endovascular treatment was aimed to address MCA occlusion and achieve recanalization distally as a first step; utilizing the Solitaire FR revascularization device. After MCA recanalization was restored, the ICA occlusion was treated with balloon angioplasty and stenting during the same setting. Results: Three patients (51-yearold male, 49 and 73-year-old females) were treated utilizing the technique described above. Near complete or complete recanalization was achieved during the procedure and confirmed with ultrasound during follow- up. Follow-up CT head revealed only small basal ganglia infarcts with sparing of the remainder of the MCA territory in all treated patients. Conclusions: The described endovascular approach above is successful and technically feasible. As the phrase "time is brain" goes, the authors suggest this approach offers earlier cerebral reperfusion and potentially saves more neuronal tissue as opposed to the reversed approach (ICA stenting followed by MCA recanalization).

\section{$\mathbf{P . 0 5 5}$}

Platelet-mediated changes to neuronal glutamate receptor expression at sites of microthrombosis following experimental subarachnoid hemorrhage

\section{JD Bell (Toronto)* H Wan (Toronto) RL Macdonald (Toronto)}

Glutamate is important in the pathogenesis of brain damage after cerebral ischemia and traumatic brain injury. Notably, brain extracellular and cerebrospinal fluid as well as blood glutamate concentrations increase after experimental and clinical trauma. While neurons are one potential source of glutamate, platelets also release glutamate as part of their recruitment which might mediate neuronal damage. This study investigates the hypothesis that platelet microthromboemboli release glutamate that mediates excitotoxic brain injury and neuron dysfunction after subarachnoid hemorrhage (SAH). We used two models, primary neuronal cultures exposed to activated platelets, as well as a whole animal subarachnoid hemorrhage preparation. We demonstrate that thrombin-activated platelet-rich plasma releases glutamate, which exceeds concentrations of 300 micromolar. When applied to neuronal cultures, this activated plasma is neurotoxic, and attenuated in part by glutamate receptor antagonism. We also demonstrate that exposure to thrombin-activated platelets induces a marked downregulation of the surface glutamate receptor GluR2, a marker of excitotoxicity exposure and a possible mechanism of neuron dysfunction. Linear regression demonstrated that seven days following $\mathrm{SAH}$ in the animal there was a strong correlation between proximity to microthrombi and reduction of surface glutamate receptors. This novel data suggests that platelet-mediated microthrombosis contributes to neuronal glutamate receptor dysfunction and might therefore influence clinical outcome following subarachnoid hemorrhage.

\section{P.056}

Prophylactic magnesium sulfate for cerebral vasospasm in aneurysmal subarachnoid hemorrhage: systematic review and meta-analysis

D Reddy (Hamilton)* A Fallah (Toronto) F Farrokhyar (Hamilton) J Petropoulos (Hamilton) RL Macdonald (Toronto) D Jichici (Hamilton)

A systematic review was conducted to determine the evidence for the use of prophylactic magnesium sulfate for the prevention of cerebral vasospasm in aneurysmal subarachnoid hemorrhage. Search methods: Medline, Embase, Cochrane library, clinicaltrials.gov and controlled-trials.com were searched with a comprehensive search strategy. 2035 records were identified in the initial search and 1574 remained after removal of duplicates. Selection criteria: Randomized, parallel group, controlled trials were included. Studies included at least one of the pre-specified outcome measures. Nine studies were included in the quantitative analysis. Results: The summary effect for GOS and MRS is a RR of 0.93 (95\% CI $0.82-1.06)$. The RR for Mortality is 0.95 (95\% CI 0.76-1.17). Delayed cerebral ischemia has a RR of 0.54 (95\% CI $0.38-0.75)$, which is the only outcome with a statistically significant summary effect measure, favouring magnesium treatment. Delayed ischemic neurological deficit has a RR of 0.93 (95\% CI 0.62-1.39). TCD vasospasm has a RR of 0.72 (95\% CI 0.51-1.03). The outcome dependent GRADE quality of evidence ranges from low to high, though is high for patient important outcomes of mortality and unfavourable functional outcome. Authors' Conclusion: Current evidence does not support the use of Magnesium Sulfate for the prophylaxis of cerebral vasospasm in aneurysmal subarachnoid hemorrhage.

\section{P.057}

Microsurgical excision and decompression of giant coiled aneurysms: report of 2 cases K Elayoubi (Montréal)* F Patrice (Montréal) MW Bojanowski
(Montréal)

Objective: Endovascular therapy with coil embolization and flow diverters for giant aneurysms may be complicated by severe mass effect. We present the surgical treatment of such complications. Material and method: Two patients underwent microsurgical excision of coils to relieve severe mass effect after endovascular treatment for giant aneurysms. The first patient is a 57-year-old women treated for a ruptured giant basilar artery aneurysm with multiple embolizations and insertion of a flow diverter. She developed a pseudobulbar syndrome followed by severe tetraparesis and respiratory distress requiring endotracheal intubation. The second patient is a 66-year-old woman who underwent endovascular treatment for a giant unruptured left MCA aneurysm with coils and flow diverter. She developed dysphasia, right hemiparesis and eventually intracranial hypertension not responding to corticosteroids and osmotherapy. Results: Both patients underwent coils excision without compromising the flow diverters. Both 
patients had significant improvement of their clinical condition with significant reduction of brain oedema surrounding the aneurysms. Conclusion: Surgical intervention of giant coiled aneurysms exerting significant mass effect is warranted in selective cases, even when faced with severe neurological impairment. The surgical pitfalls will be presented.

\section{Trauma, Critical Care}

\section{P.058}

The Mini-Mental State Examination and the Montreal Cognitive Assessment after traumatic brain injury: an early predictive study

A Alturki (Montreal)* E de Guise (Montreal) J LeBlanc (Montreal) $M$ Champoux (Montreal) C Couturier (Montreal) J Lamoureux (Montreal) M Desjardins (Montreal) J Marcoux (Montreal) M Maleki (Montreal) M Feyz (Montreal)

Background: to compare results of the Montreal Cognitive Assessment (MoCA) to those of the Mini-Mental State Examination (MMSE) in persons with traumatic brain injury (TBI) and to predict outcome at discharge from the acute care setting. Methods: The MoCA and the MMSE were administered to 214 patients with traumatic brain injury (TBI) during their acute care hospitalization in a Level I trauma center. Outcome was measured with the Disability Rating Scale (DRS). A linear regression determined that the MoCA, the MMSE, TBI severity, education level and presence of diffuse injury. Results: the model determined that the MoCA, the MMSE, TBI severity, education level and presence of diffuse injuries predicted $57 \%$ of the total variability of the DRS scores. The model without the MMSE had a $\mathrm{R}^{\wedge} 2$ of $53.7 \%$ and the model without the MoCA had a $\mathrm{R}^{\wedge} 2$ of $55.0 \%$. The models without the MMSE or the MoCA had a $\mathrm{R}^{\wedge} 2$ of $24.9 \%$. Conclusion: the MoCA is not a better predictor of outcome as assessed with the DRS than the MMSE. Perhaps it would be the case at a later time in the recovery process that is after the acute stage when cognitive deficits of patients with TBI are more subtle. Further studies on the MoCA are therefore needed in the later stages of recovery post TBI.

\section{P.059}

Hospitalized traumatic brain injury patients are susceptible to dehydration during heat waves

\section{OJ Lasry (Montréal) M Paci (Montreal)*J Marcoux (Montreal)}

Background: The literature has documented the association of elevated environmental temperatures and dehydration in disabled geriatric and psychiatric hospitalized patients. Hospitalized Traumatic Brain Injury (TBI) patients represent a disabled population that can suffer from dehydration when environmental temperatures rise. Methods: We retrospectively analyzed all patients admitted to the Neurosurgery Ward at the Montreal General Hospital with a diagnosis of Traumatic Brain Injury during the months of July and August of 2012. Seventy patients were included for whom all serum creatinine, urea and urea/creatinine ratios were collected. Subgroup analysis regarding age, comorbidities, TBI severity, need for an operative intervention and the main traumatic lesion found on head computed tomography was also completed. Results: Linear regression analysis showed a linear increase in urea/creatinine ratios when compared to rising mean environmental temperatures. This association approached statistical significance $(\mathrm{p}=0.09)$ in severe TBI patients. Furthermore, there were 14 temperature peaks over the two months. In all instances where temperature peaks lasted five days or more, severe TBI patients had a significant $(p<0.05)$ rise in their urea/creatinine ratios when compared to mild/moderate TBI patients. Conclusions: This is the first study to identify hospitalized traumatic brain injury patients as a susceptible population to dehydration. Policies and protocols instituted for other disabled populations should be adapted to hospitalized TBI patients to prevent morbidity from dehydration.

\section{$\mathbf{P . 0 6 0}$}

Decompressive craniectomy in traumatic brain injury: does size matter?

\section{P Martel (Montreal)* M Maleki (Montreal) J Marcoux (Montreal)}

Background: The neurotrauma setting, decompressive craniectomy for refractory ICP is still widely used even if the DECRA trial showed that the surgical cohort treated with bifrontotemporoparietal craniectomy was associated with more unfavorable outcomes. Likewise to the RESCUEicp trial, our institution recommends large unilateral or bilateral frontotemporoparietal craniectomy. We believe that this technique achieves better bony decompression. Recent work also suggested that craniectomy size impacts on ICP control and outcome. Methods: We identified around fifty cases of severe TBI requiring craniectomy at the Montreal General Hospital over the last ten years. We correlated the craniectomy size to different clinical variables, including ICP control, quantity of CSF removed with extra ventricular drain, ICU stay and quantity and length of post-operative hyperosmolar therapy given. Results: We project to demonstrate that cases treated with larger craniectomy size provide overall better ICP control, require less medical therapy and perhaps have a better outcome. Preliminary results show that craniectomy size varies considerably between the cases. Conclusions: We believe that large craniectomy size could be beneficial and we aim at giving intraoperative tools to assess the adequacy of the bone flap removed, since measuring the craniectomy diameter is impossible before having achieved the procedure itself.

\section{P.061}

Subgaleal hematoma in craniectomized patients: an unreported life-threatening complication of decompressive craniectomy

\section{P Martel (Montreal)* M Maleki (Montreal) J Marcoux (Montreal)}

Background: craniectomy is commonly used in acute-care neurosurgery. In fact, it is now becoming the standard of care for refractory cases of raised intracranial pressure due to severe traumatic brain injury. Many complications of this procedure are well known and treatment algorithms are already recognized. Formation of a subgaleal hematoma in such patient is a rare unreported complication. It can result in rapid neurological deterioration because the underlying brain tissue is now vulnerable and left unprotected without any rigid skull. If left untreated, such a hematoma can progress to uncal herniation. Methods: We report three cases of early and three cases of delayed subgaleal hematoma formation in craniectomized patients. We describe the circumstances 
and the steps taken in each scenario to treat this complication. Results: Several risk factors could be identified, such as coagulopathies and probably the use of low-molecular weight heparin for DVT prophylaxis. Conclusions: Subgaleal hematoma formation in a craniectomized patient is a life-threatening situation that necessitates urgent surgical attention. We propose a treatment algorithm that considers bedside reopening of the skin incision and drainage of the subgaleal hematoma in an attempt to decrease the intracranial pressure while awaiting definitive surgical treatment.

\section{P.062}

Analysis of risk factors for minor brain injury in patients presenting with a facial fracture

A Ranger (London)* A Grant (London) B Young (London) A Kirschner (London) A Yazdani (London)

Background: Minor brain injuries (BI) are increasingly recognized as an important health problem. They have received lessthan-deserved attention in the setting of facial fractures; once major $\mathrm{BI}$ is excluded, cognitive and neurological monitoring often ceases. We sought to determine prospectively the incidence of minor BI, presenting characteristics, and risk factors to determine which patients should be followed more closely. Methods: We identified 100 consecutive patients with facial fractures at a level 1 trauma center. A clinical questionnaire was used to identify those with major versus minor BI. Patients were analyzed using group comparisons of continuous data by Student's t-test and nominal and ordinal data by Pearson $\chi 2$. Logistic regression analysis was performed to identify variables associated with minor BI. Results: 38 patients met criteria for minor BI. Impaired consciousness occurred in 37. 26 and 16 reported pre- and post-injury amnesia, scoring lower on cognitive functions (orientation, concentration, short-term and delayed memory) (all p < 0.05), and standardized assessment of concussion score $(p=0.016)$. Motor vehicle collisions $(M V C)$ and combined facial fractures resulted in at least a minor BI. Mechanism of injury ( $p=0.030$ ) was the only significant predictor of minor BI, though fracture type approached significance $(\mathrm{p}=0.066)$. Conclusions: Facial fractures are frequently associated with undetected minor BI. Suspicion is especially warranted among those in MVCs or with combined facial fractures.

\section{P.063}

Does white cell count at presentation have any implication in traumatic brain injury?

WS Muhammad (Mississauga) M Qadeer (Mississauga) ME Bari (Mississauga) SA Khan (Mississauga)* I Patoli (Mississauga)

Objective: To study the association of high white cell count with severity of head injury \& outcomes in patients with isolated head injury. Methods: It was a retrospective observational study. We reviewed medical charts \& electronic database of patients with isolated head injury from 1 Jan 2006 - 31 June 2012. Demographic variables, Presentation GCS, Revised trauma score \& total white cell counts at presentation were recorded. Glasgow outcome scale was then applied on follow up. Data was analyzed on SPSS19. Results: A total of 121 patients were included in the study. Mean age of our population was 38.86 years $( \pm 16.71)$. For the purpose of analysis we divided the population into three groups on the basis of GCS \& compared for various variable $\&$ mean white cell count. Mean white cell count in mild, moderate and severe head injury was $13.81 \pm 6.12$, $18.17 \pm 5.67,19.37 \pm 7.17$ respectively with statistically significant difference between mild \& moderate head injury groups ( $p$ value $<0.001)$. We found TLC to be negatively correlated with GCS on arrival with statistical significance $(\mathrm{r}=-0.238)$. However we did not find any significant association with unfavorable outcomes or GOS. Conclusion: Total white cell count is associated with severity of head injury. However study does not find a significant association with outcomes. Studies on larger sample size is required. Key Words: Isolated head injury, Total white cell count, GCS.

\section{$\mathbf{P . 0 6 4}$}

The management of traumatic subarachnoid hemorrhage and vasospasm with milrinone

\section{OJ Lasry (Montréal)* J Marcoux (Montreal)}

Background: Traumatic subarachnoid hemorrhage (tSAH) is the most common traumatic lesion amongst traumatic brain injury patients. The literature has confirmed that cerebral vasospasm occurs at high rates in these patients and that delayed ischemic symptoms can occur and worsen patient outcomes. Unfortunately, there is limited literature on the management of post-traumatic vasospasm. Methods: We applied the "Montreal Neurological Hospital Protocol" by Angle et al, which uses Milrinone to reverse vasospasm in aneurysmal subarachnoid hemorrhage, to 3 mild TBI patients with tSAH and vasospasm that developed clinical delayed ischemic symptoms. The Modified Fisher Scale was used to grade the tSAH. Radiological confirmation with CT-Angiography was available in one patient. Results: Two of the three patients had their deficits completely reversed after Milrinone was started. The third case remained with residual neurological deficits but had an overall improvement. The Glasgow Outcome Scale for the three patients was favorable (GOS $=4-5)$. There were no reported complications from the use of Milrinone in these patients. Conclusion: The "Montreal Neurological Hospital Protocol" was successfully used in our TBI patients and appeared safe. This is the first report of Milrinone use in TBI patients for the treatment of cerebral vasospasm. This report emphasizes that larger prospective studies are warranted to establish the efficacy and safety of Milrinone for post-traumatic vasospasm.

\section{P.065}

Brainstem reversible hypertensive encephalopathy: a case report

M Labidi (Quebec)* M Dubé (Quebec) M Brisson (Quebec) M Savard (Quebec)

Background: Posterior reversible encephalopathy syndrome (PRES) is an acute neurotoxicity syndrome. Classically, MRI shows bilateral signal abnormalities in cortical and subcortical regions of the posterior circulation in relation with vasogenic edema. Methods: Case report. Results: A 48-year-old women presented following an episode of generalized seizures. She previously had progressive pancephalic headaches, intermittent confusion and disorientation. Her neurological examination revealed no focal deficit, including cranial nerves. However, her blood pressure was measured at 225/110 mmHg. An emergent MRI showed marked hyperintensity of the brainstem extending from the lower thalami to the upper cervical cord on T2WI and FLAIR sequences. With intensive 
antihypertensive therapy, her mental status considerably improved and, by day 7, she had completely recovered. On day 9, a repeat MRI showed a near resolution of T2/FLAIR hyperintensities in the brainstem and in the periventricular area. Conclusion: Patients with PRES may present with isolated brainstem edema. In these cases, there may be a "clinico-radiologic dissociation", with MRI findings far more extensive than would suggest the clinical presentation. MRI is the diagnostic tool of choice; typically, vasogenic edema with mass effect is present and T2WI, FLAIR and DWI can help differentiate PRES from ischemic, infectious, tumoral or other conditions.

\section{P.066}

Prognostic quality of MRI in severe traumatic brain injury patients

\section{G Alzhrani (Montreal)*}

There is no precise tool to accurately define the prognosis of severe TBI patients. Magnetic resonance imaging (MRI) provides more sensitive ways to define the extent of brain injury in the early phase of TBI than computed tomography (CT) do. To our knowledge, no study reported the predictive value of MRI in isolation from other important predictors of outcome such as GCS score, hypotension and pupils reactions. We conducted a retrospective study on all severe TBI patients admitted to the Montreal General Hospital who had an MRI post injury. GCS, ISS, pupil reactivity, occurrence of hypotension, age, CT scan grade, and MRI (grade and number of lesions) were collected and findings were correlated with outcome (GOS). Eighty-five patients were included. The location of lesions did not correlate with outcome. However, the number of axonal lesions had a significant relationship with the prediction of death $(\mathrm{p}=0.033)$, vegetative state $(\mathrm{p}=0.006)$, or good outcome $(\mathrm{p}=0.008)$. Furthermore, the number of lesions had a higher predictive value than GCS, hypotension or CT scan findings ( $\mathrm{p}=$ 0.002). This study infirm previous conception that brainstem lesions had a predictive value. However it provides clear relationship between the burden of axonal injury in severe TBI patients and the outcome, and this is in isolation of the other known predictors of outcome for severe TBI.

\section{P.067}

Do patients with combined traumatic brain injury and brachial plexus injury have poorer outcome than patients with an uncombined injury?

\section{Jacques (Montreal)* J Louppe (Montreal) J Marcoux (Montreal)}

Among factors affecting the outcome in brachial plexus injuries, delay to referral to surgery, cerebral plasticity, as well as cognitive possibilities in the rehabilitation stage are of utmost importance. These factors can be modified by a simultaneous brain injury, but few reports have focused on this specific issue. From our traumatic brain injury database, we performed an observational study on 22 patients, who sustained a combined brain and brachial plexus injury between the years of 2000 to 2011. Average initial GCS was 11,3. Glasgow Outcome Scale was favorable in all but one patient. Time to referral/brachial plexus surgery, brain imaging findings and neuropsychological assessment were studied. Outcome concerning the limb function was compared to a control population of brachial plexus injury patients. Cognitive changes and cerebral plasticity are discussed as possible causes of poor outcomes. We believe that cognitive rehabilitation is as important as physical therapy in this subset of patients.

\section{General Neuroradiology}

\section{P.068}

fMRI-driven DTT in the assessment of corticospinal tract in patient with glioma

X Jia (Yinchuan)* Y Yu (Yinchuan) X Wang (Yinchuan) $\mathrm{H} \mathrm{Ma}$ (Yinchuan) $Q$ Zhang (Yinchuan) $X$ Huang (Yinchuan) $H$ Xia (Yinchuan)

Background: fMRI-driven diffusion tensor tractography(DTT) has provided the ability to evaluate the spatial relationship between the corticospinal tract and tumor borders. The main objective of this study was to improve the preoperative assessment of the corticospinal fibers in patients with gliomas involving the motor cortical areas. Methods: Nineteen patients with gliomas involving the motor cortical areas underwent fMRI and DTI scan. We tracked the fibers from seed point selected in the white matter adjacent to the location of fMRI activation, and the target point was placed in the cerebral peduncle. Karnofsky performance status (KPS) was evaluated in patients before and after surgery. Results: In the seventeen patients of nineteen cases, we successfully tracked fibers by choosing seed and target point. What is more, DTT can indicate preoperatively the possibility of the extent of surgical resection. The post-operative average KPS of the 17 patients increased by more than 10 scores. Conclusions: Incorporating fMRI into DTT show a maximum benefit in surgical treatment and will enhance the accuracy of operative.

\section{DEMENTIA}

\section{P.069}

Less education predicts anticholinesterase discontinuation in dementia patients

S Saleh (Saskatoon)* A Kirk (Saskatoon) DG Morgan (Saskatoon) C Karunanayake (Saskatoon)

Objective: We investigated patient socio-demographic, clinical and functional factors predicting cholinesterase inhibitor discontinuation by patients presenting to a memory clinic in Saskatoon, Saskatchewan. Methods: Data collection began in March 2004 at the Rural and Remote Memory Clinic where family physicians referred their non-institutionalized patients. Neurological and neuropsychological assessment, patient and caregiver questionnaires provided the socio-demographic, clinical and functional variables. Univariate logistic regression analysis was used to examine possible associations between each independent variable and the binary outcome variable of treatment discontinuation. Multivariate logistic regression was used to determine predictors of cholinesterase inhibitor discontinuation within six months of drug initiation. Results: Our sample consisted of the first 63 patients $(60.3 \%$ female) for whom we prescribed a cholinesterase inhibitor. The mean age at clinic day was 74.56 years 
$(\mathrm{SD}=7.78)$. We found that years of formal education was the only variable significantly associated with cholinesterase inhibitor discontinuation by six months. The more years of formal education, the lower the rate of drug discontinuation by six months. Conclusions: Likelihood of cholinesterase inhibitor discontinuation by six months was predicted by fewer years of formal education.

\section{P.070}

\section{Benign mesial temporal lobe epilepsy}

AA AlQassmi (London)* M Jones (London) S Mirsattari (London) J Burneo (London) RS McLachlan (London)

Benign mesial temporal lobe epilepsy (bTLE) is often not well recognized. It requires at least 24 months of seizure freedom with or without antiepileptic medication. Seizure onset tends to be in adolescence or adulthood, and $40 \%$ show evidence of hippocampal sclerosis in long standing bTLE. We describe the clinical features of a benign form of temporal lobe epilepsy in 24 patients (6 Males). The mean age was 32.2 years (range 28-80), and the follow-up period exceeded two years. Neurological examinations were performed at every follow up visit (4-12 months). All patients had at least two EEGs and a MRI. All patients had a mild epileptic disorder and achieved seizure freedom at onset of treatment, which persisted for at least 24 months. Sixteen patients (73\%) had MTS, 79\% had significantly older age at onset, and two patients (8\%) had early onset. EEGs were normal in four, showed temporal slowing in 17 and temporal spikes in three. Aetiology consisted of a viral encephalitis in one, but was unknown in 23. In three patients, we attempted discontinuation of AEDs after a long period of remission (5-8 years), but all had recurrence within two to four weeks. Not all temporal lobe epilepsy, even with MTS, is refractory to medication. Lifelong treatment is advocated.

\section{General Neurology}

\section{P.071}

Inflammatory focal myositis with IgG4 infiltrate in a patient with rheumatoid arthritis: a case report

MC Zlatescu (London)* M Alturkustani (London) LC Ang (London) AE Thompson (London) SL Venance (London)

Background: IgG4-related disease (IgG4-RD) is a recently defined entity with multi-organ involvement characterized by serum IgG4 elevation and tissue IgG4-positive plasma cell infiltration. The literature suggests IgG4-related autoimmune pancreatitis as the prototype, however other commonly affected sites are the digestive tract, salivary gland, orbit, lymph nodes, and retroperitoneum. To our knowledge there is no description in the literature of $\mathrm{IgG} 4$ infiltrates in muscle. Methods: We describe a 64-year-old female with stable rheumatoid arthritis treated with golimumab. She presented with new onset of bilateral temporalis muscle pain, tightness around her temples and focal tenderness and firmness on palpation of the muscle. CK and EMG were normal. Results: Histopathologically, a temporalis muscle biopsy revealed extensive inflammatory reaction with severe loss of myofibers, marked fibrosis, lymphoplasmacytic aggregates and prominent $\mathrm{IgG} 4$ plasmacytic infiltrate ( $>10$ IgG4 per high-power field) in addition to elevated serum levels of IgG4. Clinically the patient improved over
6 months without any additional immunomodulation. Conclusions: This unusual case of inflammatory focal myositis with involvement of temporalis muscle extends the spectrum of IgG4-RD. Further studies are necessary to delineate the role of $\mathrm{IgG} 4$ in the pathogenesis. However, physicians need to be aware of this entity as biopsy and serum IgG differential are critical in making the diagnosis.

\section{P.072}

Cognitive function in cerebellar ataxia: an assessment of MSA$\mathrm{C}$ and sporadic ataxia

\section{P Jacova (Vancouver) SD Spacey (Vancouver)*}

Background: The integrity of cognitive function in Multiple System Atrophy Cerebellar Variant (MSA-C) and Sporadic Ataxia (SA) is uncertain. We used the Montreal Cognitive Assessment (MoCA) to evaluate cognitive function in these disease groups. Methods: Subjects and age matched controls were recruited at the UBC Neurogenetics Clinic. History of neurodegenerative diseases other than MSA-C and SA were exclusion criteria. The MoCA and International Cooperative Ataxia Rating Scale (ICARS) were administered. Disease groups were each compared to controls using Mann-Whitney U Test. Correlations of MoCA with ICARS were examined with Spearman rank coefficient. Results: There were 6 subjects with MSA-C $(\mathrm{M}$ age $=64.2 \pm 9.2,4 \mathrm{M} / / 2 \mathrm{~F}, \mathrm{M}$ disease duration $(\mathrm{DD})=4.8 \pm 2.2), 14$ with $\mathrm{SA}(\mathrm{M}$ age $=61.4 \pm 7.0,7 \mathrm{M} / / 7 \mathrm{~F}, \mathrm{M}$ $\mathrm{DD}=11.9 \pm 5.7)$ and 14 controls $(\mathrm{M}$ age $=57.0 \pm 8.2,7 \mathrm{M} / / 7 \mathrm{~F})$. MoCA scores were significantly lower for MSA-C and SA $(M=19.0 \pm 3.5$, $\mathrm{p}=0.000, \mathrm{M}=23.4 \pm 3.7, \mathrm{p}=0.004$, respectively), compared to controls $(\mathrm{M}=27.4 \pm 1.3)$. The MSA-C group scored significantly lower than the SA group $(\mathrm{p}=0.033)$. MoCA scores were inversely correlated with ICARS for MSA-C ( $\mathrm{r}=-0.250, \mathrm{p}=0.633)$ and for controls ( $\mathrm{r}=-$ $0.463, \mathrm{p}=0.095)$ but not for SA $(\mathrm{r}=0.003)$. Conclusion: This study demonstrates early, moderate cognitive impairment in MSA-C. By contrast, in SA cognitive impairment appears to be milder, later and unrelated to cerebellar ataxia severity.

\section{P.073}

C-ANCA associated vasculitis presenting as isolated focal leptomeningeal enhancement: report of a case and review of the literature

M Gagné Brosseau (Sherbrooke)* S Gosselin (Sherbrooke) F Evoy (Sherbrooke)

We report the case of a 63-year-old Caucasian man who presented with a five-month history of stereotyped episodes of left perioral tingling spreading to the left hand over a few seconds, sometimes associated with left lower face asymmetry, dystonic position of the left fingers and heaviness in the left arm. His past medical history included bilateral carotid endarterectomy and right common carotid artery stenting many years ago for bilateral transient ischemic attacks as well as renal angioplasty, high blood pressure, dyslipidaemia and coronary artery disease. Brain magnetic resonance imaging (MRI) demonstrated right frontoparietal leptomeningeal enhancement with adjacent cortex signal abnormality. A pancorporal positron emission tomography (PET) scan showed hypermetabolism in the right frontoparietal region and no other hypermetabolic zone. Two electroencephalograms (EEGs) performed during hospital stay were normal. Extensive serologic 
investigation revealed isolated positive cytoplasmic anti-neutrophil cytoplasmic antibodies (C-ANCA). Meningeal biopsy demonstrated inflammation with abundant lymphocytes, compatible with leptomeningeal vasculitis. Levetiracetam $500 \mathrm{mg}$ twice daily was started for the probable epileptic nature of the episodes. Also, Prednisone 50mg once daily was prescribed and within four weeks, the enhancement almost completely resorbed and the episodes stopped. This case illustrates the rare but possible limited form of ANCA-associated vasculitis.

\section{P.074}

Familial hemiplegic migraine presenting with encephalopathy and seizures

\section{A Jahangirvand (Saskatoon)* W Fitzpatrick (Saskatoon)}

Background: We report a case of familial hemiplegic migraine type 1 (FHM-1) presenting with encephalopathy, hallucinations and new onset focal seizures. Method: Case report Results: A 26-yearold female with long-standing history of presumed FHM-1 presented to hospital with right temporal headache accompanied by visual aura and left hemiparesis. Three days after onset she became encephalopathic with profound visual and auditory hallucinations. She also developed recurrent episodes of focal motor seizure activity, with occasional secondarily generalized tonic-clonic seizure. Laboratory analysis and lumbar puncture was unremarkable. Brain CT and MRI revealed dramatic diffuse right hemisphere cerebral edema. EEG showed profound right hemisphere delta activity and occasional epileptiform discharges. Keppra and acetazolamide were initiated and she gradually returned to baseline within 14 days. Repeat MRI in one month revealed complete resolution of cerebral edema. Genetic testing identified the responsible CACNA1A gene mutation. Discussion: Hemiplegic migraine is a rare form of migraine with motor aura. It can occur as a sporadic or familial disorder. FHM-1 is the most common familial form, caused by mutation in the CACNA1A gene. Weakness can last days. Although uncommon, patients are at risk of major episodes of encephalopathy and severe attacks can include seizures. During an attack imaging may reveal ipsilateral cerebral edema and EEG may show diffuse slow waves contralateral to the motor deficit.

\section{P.075}

\section{Altered mTOR signaling and mitochondrial respiration in the} BTBR mouse model of autism

$Y$ Ahn (Calgary)* $M$ Narous (Calgary) J Smith (Calgary) $R$ Tobias (Calgary) SA Masino (Hartford) JM Rho (Calgary)

Background: BTBR T+tf/J (BTBR) mice exhibit core features of autistic spectrum disorder (ASD) including: abnormal social interactions, cognitive inflexibility and repetitive motor behaviors. While the pathogenesis of ASD remains obscure, the mammalian target of rapamycin (mTOR) signaling pathway is increasingly being implicated. As such, we hypothesized that the mTOR pathway might be impaired in BTBR mice. Methods: Western Blot: Lateral temporal neocortices were collected from P35 BTBR mice and probed with antibodies against P-AKT, P-mTOR, P-S6, and P4EBP1. Mitochondrial Bioenergetics: Total mitochondria were isolated from neocortex of similarly aged BTBR and B6 mice. Bioenergetic profiles were established using a standard Oxytherm and the Seahorse Bioscience XF24 Flux Analyzer. Results: In BTBR mice $(n=3)$, mTOR signaling phospho-proteins were significantly decreased compared to B6 controls $(n=5 ; P<0.05)$. Further, BTBR mice demonstrated increased basal respiration compared to B6 animals ( $\mathrm{n}=4$ per group, $\mathrm{P}<0.05)$ and increased oxygen consumption rates (OCR) $(\mathrm{n}=7$ per group, $\mathrm{P}<0.001)$. Conclusions: Our results provide additional evidence that alterations in mTOR signaling may play a role in the pathogenesis of ASD. Additionally, we show intrinsic enhancement of mitochondrial respiration, which may reflect a compensatory mechanism. These results suggest a mechanistic connection between mTOR signalling and mitochondrial function in BTBR mice.

\section{P.076}

\section{Infiltrative brain tumor mimicking acute viral encephalitis}

A Jahangirvand (Saskatoon)* AP Masiowski (Saskatoon) S SavediaCayabyab (Saskatoon) A Rajput (Saskatoon)

Background: We report a case of an infiltrative anaplastic astrocytoma presenting as acute encephalitic illness. Method: Case report Results: A previously healthy 49-year-old gentleman presented to hospital upon waking in the morning with sudden onset of headache, mild fever, and confusion. Initial brain MRI revealed increased signal in the temporal lobes with no enhancement or mass effect. Cerebrospinal fluid showed normal cells and chemistry with elevated opening pressure. The patient initially improved after medical treatment with a presumed diagnosis of herpes simplex encephalitis (HSE). Extensive testing did not reveal a viral source and detailed investigation for paraneoplastic and other autoimmune conditions was negative. There was no relief with trial of steroids or IVIG and a ventriculoperitoneal shunt was eventually placed to manage high-pressure headaches. After 3 months, the patient was readmitted with new onset focal seizures. A follow-up brain MRI revealed marked increase signal change and new corresponding diffusion restriction. Stereotactic brain biopsy revealed an infiltrative anaplastic astrocytoma. Discussion: HSE is the major cause of sporadic encephalitis with a predilection for the temporal lobe. While the initial presentation was consistent with HSE, it can be difficult to distinguish an infiltrative temporal lobe lesion early on. If definitive diagnosis using PCR assays for common viruses cannot be found, a stereotactic brain biopsy should be considered in patients with temporal lobe lesions.

\section{P.077}

\section{Insulin neuritis: an unusual form of diabetic polyneuropathy}

LW Ferguson (Saskatoon)* C Voll (Saskatoon)

Background: We report a case of suspect "insulin neuritis, an acute painful polyneuropathy which may complicate rapid correction of hyperglycemia with insulin. Methods: Case report Results: A 74-year-old male was referred to the neurology service with rapidly progressive weakness and dysesthetic extremity pain. He had known type 2 diabetes mellitus, managed with diet only prior to admission. He was admitted to hospital with pneumonia and subcutaneous insulin was prescribed to manage uncontrolled severe hyperglycemia. He developed a rapidly progressive quadriparesis with diffusely painful extremities within three days of insulin administration. Sensorium, cognition and cranial nerve examinations were normal. His motor exam was limited by pain with tactile stimulation of the extremities and with movement of the 
limbs. Upper and lower extremity strength was graded as $2 / 5$ proximally and distally. Biceps and triceps reflexes were hypoactive (1+) while knee and ankle were absent. Plantar reflexes were mute. Pain and vibration sensation was impaired below the knees bilaterally and proprioception was impaired with distal lower extremities. Nerve conduction studies showed absent motor and sensory responses in upper and lower extremities. Conclusions: This case highlights an under-reported rare form of diabetic neuropathy. While appropriate glycemic control is important, overly rapid correction with insulin may lead to a severe painful polyneuropathy, sometimes termed "insulin neuritis".

\section{P.078}

\section{A rare case of MAMA}

\section{White (Saskatoon) C Gervais (Saskatoon)*}

Background: We report a case of Multifocal Acquired Motor Axonopathy (MAMA) in a young woman. Methods: Case report Results: Our patient is a 35-year-old caucasian female. She experienced onset of symptoms at 21 . They involved slowly progressive muscle wasting \& weakness of finger extension in the fingers of her right, then left hand. It gradually spread up both arms, involving biceps, triceps, infraspinatus and deltoids bilaterally. Two inital sets of EMG/NCS demonstrated a multifocal non-myotomal motor axonal process involving denervation without conduction block in the arms. At age 32 she noticed weakness of right ankle dorsiflexion. Over the past three years her weakness has spread to bilateral hip abduction, ankle dorsiflexion \& ankle eversion. Reflexes are absent in upper limbs, 3/4 at the knees, 2/4 at ankles, with downgoing plantars. She has no associated sensory, respiratory, bladder, or bulbar symptoms. Comorbidities include Psoriasis, Raynaud's Phenomenon \& Crohn's disease. MRI Brain/Spine, LP, Anti-GM1 antibodies, Lyme Serology and Genetic testing for SMA are unremarkable. She failed to respond to trials of IVIG, Prednisone \& Imuran. MAMA is a rare entity in the literature, and report of these cases is important in developing better identification and understanding of disease.

\section{P.079}

\section{Anti-NMDA-receptor encephalitis: case series and analysis}

\section{RR Magun (Hamilton)* DJ Sahlas (Hamilton)}

Objective: We have performed a Case Series analysis of recent admissions to McMaster University Hospitals of paraneoplastic encephalitis due to antibodies against the Anti-N-methyl-Daspartate receptor (anti-NMDA-R). Background: Anti-NMDAreceptor encephalitis is an immune-mediated syndrome that is increasingly being recognized cause of seizure or psychiatric conditions requiring hospital admission. Previous reports have described young females presenting with symptom of personality changes, focal neurological deficits, seizures, or autonomic dysfunction. In the past it has often reported in patients with ovarian teratomas. Design/Methods: Case Series Analysis Results: We describe an array of clinical presentations of anti-NMDA-R encephalitis over the past several years at McMaster University. Complex partial seizures, personality change, and psychiatric symptoms were common presentations of this disorder. A majority of patients showed dramatic improvement with five day course of IVIG at $2 \mathrm{~g} / \mathrm{kg}$. Many of the patients had no underlying tumour identified at time of presentation. Conclusions: These findings indicate antibody-associated immune response against NR1/NR2 heteromers of the NMDA receptor (NMDAR) is a potentially severe yet reversible medical condition. Often it may precede the detection of any tumor such as ovarian teratoma by several years and therefore follow-up screening is indicated.

\section{Multiple Sclerosis}

\section{P.081}

\section{Clinical MRI correlation in RRMS patients on natalizumab}

MC Zlatescu (London)* SA Morrow (London)

Background: Several studies have evaluated the efficacy of natalizumab in relapsing remitting MS (RRMS) patients but clinical and neuroradiological correlation has been insufficiently studied. Methods: We retrospectively reviewed the clinical course and MRI results in patients treated with natalizumab. The main parameters included the clinical course and the number and volume of Gadolinium-enhancing, new or enlarging T2 hyperintense on natalizumab. Results: Thirty five patients were reviewed. While on natalizumab, 19/35 patients had 0 relapses, 10/35 1 relapse, 1/35 2 relapses and 5/35 demonstrated continuous progression. Of the 11 relapses, 2 correlated with new MRI lesions; 2 relapses were optic neuritis and another transverse myelitis and thus may not have been visualized radiologically, leaving $6 / 11$ relapses without corresponding MRI activity. Overall, new MRI lesions were detected only in 6/35 (17\%) patients. EDSS scores improved or remained stable while on natalizumab in $24 / 35$ (68\%) patients. Of the patients who evolved to secondary progressive MS (SPMS), 4/5 did not show any inflammatory activity on the MRI, while $1 / 5$ had multiple new lesions over time with continuous EDSS progression (no clear relapses). Conclusions: Our review demonstrates that natalizumab suppresses both clinical and MRI activity in RRMS but that MRI activity does not always predict clinical activity. However, progressive worsening of clinical symptoms in the absence of MRI activity is suggestive of a conversion to SPMS.

\section{P.082}

Treatment of natalizumab-associated PML-IRIS - a case report $N$ Parks (Halifax)* $T$ Campbell (Halifax) C Maxner (Halifax) $V$ Bhan (Halifax)

Background: Nearly all cases of progressive multifocal leukoencephalopathy (PML) among multiple sclerosis (MS) patients treated with natalizumab develop immune reconstitution inflammatory syndrome (IRIS). Treatment for natalizumabassociated PML-IRIS is not well established. Suppression of the immune response by corticosteroids for IRIS may promote PML progression. Maraviroc, a CCR5 inhibitor with immunomodulating properties, may be effective in preventing IRIS. Methods: We describe a case of natalizumab-associated PML-IRIS treated with maraviroc and methylprednisolone. Results: A 50-year-old man with relapse-onset MS treated with natalizumab for five years presented with subacute onset of progressive cognitive impairment. PML was diagnosed following detection of a nonenhancing lesion in the left temporal subcortical white matter on MRI and JC virus in the 
cerebrospinal fluid. Natalizumab was removed by plasma exchange and he received mirtazapine (in vitro activity against JC virus). Maraviroc $300 \mathrm{mg}$ PO bid was prescribed for prevention of IRIS. However, IRIS was diagnosed three weeks later, with classic MRI findings and accompanying clinical worsening (marked aphasia and right arm paresis). He received intravenous methylprednisolone followed by a slow taper of oral prednisone. Subsequently, he demonstrated significant clinical improvement. Conclusions: Natalizumab-associated PML-IRIS may develop despite prophylactic treatment with maraviroc while corticosteroids retain a role in treatment of IRIS.

\section{General/Intracranial NeUrosurgery}

\section{P.083}

Aneurysmal bone cyst of the temporal bone presenting with headache and partial facial palsy

SN Kletke (Hamilton)* A Algird (Hamilton) A Alobaid (Hamilton) KK Reddy (Hamilton)

Aneurysmal bone cysts are benign bony lesions that rarely affect the skull base. We describe a case of an otherwise healthy 23-yearold woman who presented with a pulsatile noise in the left ear, followed by a four-week history of throbbing headache with nausea. There was no associated emesis, visual or auditory changes, or other neurological features. Neurological examination revealed a partial left lower motor neuron facial palsy. Computed tomography and magnetic resonance imaging demonstrated a large lesion in the left middle cranial fossa skull base with erosion of the petrous temporal bone. Based on preoperative imaging studies, the lesion was interpreted to likely be consistent with a meningioma. An orbitozygomatic approach was utilized for tumor resection. Pathological evaluation demonstrated evidence of an aneurysmal bone cyst. Postoperatively, the patient had improvement in the lower motor neuron facial paresis and there was no worsening of hearing. She had no other new deficits and was discharged home on the third post-operative day. A thorough literature search identified a few case descriptions of aneurysmal bone cyst in this location. The authors reviewed all available literature and discuss the clinical significance and management of this lesion.

\section{P.084}

Rate of intraventricular hemorrhage post-ventricular peritoneal (VP) shunt insertion

AO Alobaid (Hamilton)* MR Bennardo (Hamilton) JK Multani (Hamilton) E Kachur (Hamilton) A Cenic (Hamilton)

Background: We determined the rate of intraventricular hemorrhage (IVH) and intracranial hemorrhage (ICH) post-VP shunt surgery since current literature on this is lacking. Our objective is to determine the rate of IVH and ICH from post-op CT radiological reports after primary insertion or first proximal revision. Methods: We carried out a retrospective analysis of medical records from VP shunt patients at Hamilton General Hospital from 2006 to 2012. Statistical analysis was done using SPSS 19. Results: A total of 277 patients were identified. Of these, 122 cases included primary VP insertions and 27 cases included first revisions for a total of 149 cases. The mean age at VP shunt operation is 49 years. Thirty-four percent $(n=51)$ of patients had post-op hemorrhages following VP shunt surgery (19.5\% IVH, $8.7 \%$ $\mathrm{ICH}$ and $6.0 \%$ both). The rate of IVH post-operatively for primary or revision surgery was $22 \%$ and $42.3 \%$, respectively. The rate of IVH between these two surgeries was found to be significantly different $(\mathrm{p}=0.046)$. All other comparisons were found to be nonsignificant. Conclusion: We report here the rate of IVH and ICH post-VP shunt surgery. The rate of IVH is significantly higher for VP shunt revisions when compared to primary surgeries.

\section{P.086}

Programmable valve in the treatment of secondary tonsillar herniation due to lumboperitoneal shunt

FB Maroun (St. John's)* D Buckley (St. John's) R Avery (St. John's)

Background: When severe, the treatment of idiopathic intracranial hypertension includes, among other modalities, the insertion of a lumboperitoneal shunt. Tonsillar herniation seen from over drainage is frequently asymptomatic. Method: We present a case of an 18-year-old male who was diagnosed with benign intracranial hypertension at the age of eight years. Because of severe papilledema, headache and 6th nerve palsy, an insertion of lumboperitoneal shunt was carried out with resolution of his symptoms and signs. Ten years later he presented with a four month history of neck pain and occipital headaches. His neurological examination was normal. MRI showed a marked tonsillar herniation with syringomyelia and edema of the upper cervical cord. The lumboperitoneal shunt was revised and a programmable valve was inserted at 2.0. Improvement of symptoms was noted immediately after. Conclusion: Symptomatic tonsillar herniation after lumboperitoneal shunt can be treated in a less aggressive manner by an insertion of a programmable valve controlling CSF outflow rather than posterior fossa decompression or ventriculoperitoneal shunt.

\section{P.087}

Patients' anxiety around incidental MRI findings: a qualitative study H Jagadeesh (Toronto)* M Bernstein (Toronto)

Background: Incidental findings are a common on MRI. Our study examined how patients are told about their incidental finding, anxiety until the neurosurgical consultation, and afterward. Methods: Qualitative research methodology was used. Thirty-two participants were interviewed using open-ended questions. Answers were transcribed and analyzed for themes. Results: The level of patient satisfaction for the initial breaking of the news averaged 4.1 (range 1-5). Four themes were identified: 1) Emotional stresses over incidental findings are partially dependent on how the news was communicated; 2) Breaking worrisome news is best done in person, but telephone communication can sometimes be acceptable; 3) Patients are divided about how much information they wish to get about incidental findings before going for a MRI; 4) Waiting for the neurosurgical consultation is a stressful time without adequate support. Conclusions: When dealing with an unexpected MRI finding, patients are anxious about the situation. Our study exposes ways the experience could be made more comfortable for patients right from the start, from being told the news in a calm and 
sympathetic manner, to providing support for patients while they wait for a meeting with a neurosurgeon, to expediting the neurosurgical consultation.

\section{P.090}

\section{Microvascular decompression of the optic nerve}

A Jack (Edmonton)* CJ O'Kelly (Edmonton) MM Chow (Edmonton)

Background: Intracranial dolichoectasia affects primarily elderly patients with vascular risk factors. Reported rates vary between 0.06-5.8\%, however most remain asymptomatic. Symptomatic compression of the optic apparatus by a tortuous internal carotid artery (ICA) is exceedingly rare. Reported here is a patient who presented with a visual field deficit due to supraclinoidal carotid artery compression of the right optic nerve. Case Presentation:A 36year-old woman presented with onset of a right monocular nasal visual field deficit. Laboratory and ophthalmological work-up revealed no discernible cause, however magnetic resonance imaging (MRI) and angiography demonstrated distortion of the right optic nerve by the ipsilateral supraclinoidal ICA. A pterional approach for craniotomy was proposed for microvascular decompression. Intraoperatively, the optic nerve and ophthalmic segment of the carotid were tethered by arachnoidal adhesions. Following surgery, untethering and microvascular decompression of the nerve proved to result in visual field improvement. Conclusion: Visual field loss due to optic nerve compression from an ectactic carotid artery is rare, however should be considered in the absence of more common causes. Here, microvascular decompression was performed with a modest improvement in visual field testing.

\section{P.091}

Predicting success of endoscopic third ventriculostomy on clinical grounds: validation of the ETV success score in a "closed-skull" population

$M$ Labidi (Quebec)* G Lapointe (Quebec) P Lavoie (Quebec) $S$ Obaid (Montreal) A Weil (Montreal) M Bojanowski (Montreal) A Turmel (Quebec)

Introduction: Endoscopic third ventriculostomy (ETV) has become the first line of treatment in obstructive hydrocephalus. The Toronto group (Kulkarni et al, 2009) developed the ETV Success Score (ETVSS) to predict the clinical response following ETV based on age, previous shunt and hydrocephalus etiology in a pediatric population. However, the use of the ETVSS has not been validated for a population comprising adults. Objectives: The objective of this study is to validate the ETVSS in a "closed-skull" population, including patients aged two years or older. Methods: In this retrospective observational study, medical charts of all consecutive cases of ETV performed in two university hospitals were reviewed for demographic and clinical variables. The ETVSS was calculated for all patients. Discriminative properties along with calibration of the ETVSS will be established for our population. Results (preliminary): 197 primary ETVs were incuded in our study. The mean age of was 41 years ( 3 years to 85 years old). ETV was successful at 6 months in 159 patients $(80.7 \%)$. Neither age nor previous shunt were significantly associated with unsuccessful ETV. However better outcomes were achieved in patients with aqueductal stenosis, tectal compressions and other tumoral hydrocephalus than in cases secondary to myelomeningocele, infection or haemorrhage $(p=0.03)$. Conclusion: Etiology may be the predominant prognostic factor in adults and children with closed fontanels undergoing ETV.

\section{P.092}

\section{Peripheral nerve sheath tumor: dealing with the unexpected}

\section{Jacques (Montreal)* M Hebert-Blouin (Montreal)}

Neurofibromas and schwannomas are the most common peripheral nerve sheath tumour encountered. They are well defined and progress very slowly. Imaging follow-up is being done annually, but immediate intervention should be considered if the mass is continuing to grow, becomes painful and or is associated with neurological deficit. The radiological criteria are well known, but in certain cases, imaging fails to reveal the nature of these lesions and therefore unforeseen pathology can be anticipated. We will discuss five patients who presented with presumed benign peripheral nerve sheath tumours clinically and confirmed with imaging. When in fact these patients were later found to have the following pathologies; leimysarcoma, malignant transformation of a neurofibroma, synovial sarcoma, angiolymphoid hyperplasia and a castleman syndrome. The Intraoperative and postoperative strategies will be reviewed as well as their outcome so far.

\section{SPINE}

\section{P.093}

Complications in halo vest treatment of cervical spine injuries $R$ Nguyen (Calgary)* S Casha (Calgary) RJ Hurlbert (Calgary)

Background: Halo vest immobilization (HVI) is the gold standard for external fixation of the cervical spine. Numerous studies cite high rates of HVI complications including infections, pneumonia, and non-union. Compared to other centers, our center has orthotists that apply all halo braces. Hypothesis: Application of halo vests in a standardized manner results in higher success and lower complication rates. Methods: Retrospective chart review of cervical spine traumas requiring HVI presenting to the Foothills Medical Center, Calgary, between 1999 and 2012. Results: 900 patients were identified. To date, 148 patients have been analyzed. 128 patients required HVI only; 20 patients required halo and surgical fixation. Seven failed HVI requiring further surgical management. Medical complications include pin site infections (16.2\%), pneumonia (13.5\%), dysphagia (6.8\%), and DVT(2\%). Discussion: HVI is a common treatment for cervical spine injuries. High complication rate has deterred many from considering its use. One of the most common medical complications of HVI is pin-site infections, and reported to be between $8-36 \%$. The failure rate of HVI is reported to be as high as $20-30 \%{ }^{1}$. In a center where halo vests are placed by experienced orthotists with regular follow-up, the medical complication rate is consistent with the literature. However, the success of halo vest treatment is significantly higher.

\section{REFERENCE}

1. Bransford, RJ et al. Halo vest treatment of cervical spine injuries. Spine 2009;34 (15): 1561-6. 


\section{P.094}

Can intraoperative monitoring predict post-operative course in extra-medullary spinal tumor?

\section{IM Alnaami (Edmonton)* $S$ Husak (Edmonton) $J$ Norton (Edmonton)}

Background: Intraoperative monitoring (IOM) is been used over the last couple of decades to guide extend of surgical resection and avoid major neurological deficit. Very little is known whether IOM can predict post-operative course of patients undergoing extramedullary spinal tumor resection. Method: Eight patients underwent surgical resection of intradural extra-medullary spinal tumors under IOM. All patients are followed up in clinic. Results: Nine patients (five males and four females) with age range 16-78 years are included. The tumors include six schwannomas, one meningioma, one lipoma, and one myxopapillary ependymoma. IOM showed a mean reduction in latency of the motor evoked potential (MEPs) intraoperatively of $20 \mathrm{~ms}$, with around $2 / 3$ of this reduction occurring upon the immediate debulking, and the remaining up until the time of skin closure. Conclusions: IOM can be utilized not only as an adjunct to guide surgical resection of extra-medullary spinal tumor, in addition; it can be a helpful tool to predict post-operative course and potentially the speed of recovery.

\section{P.095}

Spinal subdural hematoma in the setting of atlanto-occipital dislocation: the case for early aggressive management

Z Tymchak (Saskatoon)* M Kelly (Saskatoon) A Woo (Saskatoon) K Meguro (Saskatoon)

Background: Traumatic spinal subdural hematomas (SSDH) are rare. Few cases have been reported in the setting of atlanto-occipital dislocation. Methods: We present the case of a 31-year-old gentleman who sustained polytrauma from a motor vehicle accident. He presented with partial loss of brainstem reflexes and hemodynamic instability, yet was able to follow simple commands. Imaging revealed atlanto-occipital subluxation and SSDH extending from the foramen magnum to $\mathrm{C} 4 / 5$. There was little to no supratentorial pathology. The patient was treated with an occiput-C4 decompression and fusion and evacuation of the SSDH. Results: The patient underwent an extended stay in hospital but was eventually weaned from his tracheostomy and has since been transferred to our rehabilitation centre. He was able to communicate verbally and displayed good cognitive capacity. Conclusions: Patients suffering traumatic atlanto-occipital injuries with or without SSDH often die prior to reaching medical care. Our experience suggests that survivors should be managed aggressively regardless of their clinical presentation. This is particularly true in those patients with injuries confined to the cervico-medullary region as they may have better functional outcomes given the relative sparing of the higher cortical centres.
P.096

Familial lumbar spondylolysis and spondylolisthesis

FB Maroun (St. John's)* A Engelbrecht (St. John's) V Sahajpal (St. John's) TG Hogan (St. John's) B Fernandez (St. John's)

Background: The etiology of idiopathic spondylolysis and listhesis is unclear. The possibility of a genetic cause has been incriminated. Method: We report a large family with five members including a mother, father and three children, who have been diagnosed with spondylolysis and associated spondylolisthesis at the first lumbar and sacral segments. They all presented with back pain and some with sciatica requiring surgical intervention in three children. Clinical, radiological and operative findings will be presented. Conclusion: The occurrence of this anomaly affecting the parents and the three children, clearly raises the possibility of an autosomal dominant inheritance. A detailed genetic study is being assessed.

\section{Pediatrics (NeUrology, Neurosurgery)}

\section{P.097}

The epidemiology of chronic and intermittent ataxia in children in Manitoba, Canada

M Salman (Winnipeg)* E Lee (Winnipeg) A Tjahjadi (Winnipeg) B Chodirker (Winnipeg)

Background: Chronic ataxia is caused by several disorders. The epidemiology of chronic ataxia has not been studied systematically. Our aim was to determine the epidemiology of pediatric chronic ataxia in Manitoba. Methods: Multiple sources and disease codes were used to identify children (0-16 years old) with chronic ataxia (>2 months duration) seen at Winnipeg Children's Hospital during 1991-2008. Patients with tumors, isolated vestibular or peripheral nerves diseases were excluded. Results: We identified 184 patients (mean age (SD) 15 (7.7)y, M=F) with ataxia. Median age at the presenting symptom onset was 1.25 years and at axia onset 3.1 years. Median duration of follow-up was 6.4 years. During the study period, the crude incidence rate was 5.62/10000; the crude prevalence rate was 6.62/10000; and the crude mortality rate $0.446 / 10000$. The commonest presenting symptoms were developmental delay, ataxia, or seizures. The most common diagnoses (known in 129) were Angelman syndrome $(\mathrm{N}=16)$, ataxia telangiectasia $(\mathrm{N}=13)$, mitochondrial disease $(\mathrm{N}=9)$, Friedreich ataxia $(\mathrm{N}=7)$, stroke $(\mathrm{N}=7)$, and familial/genetic episodic ataxia $(\mathrm{N}=7)$. Conclusions: Chronic ataxia is a relatively common earlypresenting symptom in childhood. A specific diagnosis is possible in $70 \%$ of patients. The mortality rate is relatively low and the disease burden is high with significant co-morbidities e.g. developmental delay and epilepsy. 
P.098

The syndrome of infantile-onset saccade initiation delay (congenital ocular motor apraxia)

M Salman (Winnipeg)* K Ikeda (London)

Objective: Infantile-onset saccade initiation delay (ISID) is a defect in initiating volitional saccades. Several abnormalities are reported in ISID including developmental delay. Our aim was to quantify these abnormalities. Methods: Detailed review of the English medical literature revealed 383 patients with possible ISID. Patients with inadequate information, Joubert syndrome, neurodegenerative disorders or acquired SID were excluded whenever possible. Details on the remaining 325 patients were analyzed. Results: Head thrusts were reported in $80.6 \%$ of patients. Spontaneous saccades were present in $17.2 \%$. Impaired smooth ocular pursuit was reported in $37.5 \%$, strabismus in $15.7 \%$, and nystagmus in $10.2 \%$. Deficits in the fast phases of the vestibuloocular reflex and optokinetic response were reported in $41.2 \%$ and $72.6 \%$ respectively. Hypotonia was reported in $38.8 \%$, developmental delay in $45.2 \%$, speech/language delay in $41.2 \%$, and ataxia/clumsiness in $53 \%$. Neuroimaging $(\mathrm{N}=234)$ was normal in $32.9 \%$. Infratentorial abnormalities were reported in $40.2 \%$ and cerebrum in $18.8 \%$. Analysis on 288 patients using stricter inclusion criteria had minimal impact on the results. Conclusions: Significant abnormalities are associated with ISID. This suggests that ISID is a neurological syndrome rather than primarily a disorder of saccadic initiation. The high prevalence of various abnormalities in these patients is consistent with widespread brain dysfunction.

\section{P.099}

Comparison between radiologic feature of infantile glioblastoma and desmoplastic tumors: BC Children's Hospital (BCCH) experience and review of literature

\section{AA Bader (Vancouver)*}

Background: On standard MRI scans, two of the commoner supratentorial infantile tumours, glioblastoma (GBM) and the benign desmoplastic infantile glioma (DIG) may look similar. We hypothesized that diffusion MRI would show restricted diffusion in infantile GBM but not in DIG, thus distinguishing these 2 tumours. Methods: Retrospective review of MRI features of infantile GBM and DIG at BCCH between 1982-2012, supplemented by literature review of MRI characteristics of these tumours. Results: Of 70 infantile brain tumors at BCCH, 2 GBM and 3 DIG met inclusion criteria. All cases were supratentorial, with cystic-solid consistency. On diffusion MR studies, both GBM but none of the DIG had restricted diffusion. Literature review revealed 30 cases of infantile GBM and 42 cases of DIG with MRI findings described. The tumor was cystic-solid in $33 \%$ of GBM and $80 \%$ of DIG. Enhancement was heterogeneous in $30 \%$ of GBM cases and $20 \%$ of DIG cases. Diffusion MRI studies in 2 published cases of infantile GBM had restricted diffusion. Only 1 report described diffusion scans in DIG, but interpretation was difficult. Conclusions: Although reported rarely in the literature, diffusion MRI seemed to distinguish between infantile GBM and DIG better than other radiographic parameters.

\section{P.100}

Chemotherapy-induced peripheral neuropathy among pediatric oncology patients

MJ Purser (Ottawa)* D Johnston (Ottawa) H McMillan (Ottawa)

Background: Vinca alkaloids and platinum-containing chemotherapeutic drugs have the potential to cause chemotherapyinduced peripheral neuropathy (CIPN). This study was to determine the frequency of CIPN among children who were treated for ALL, lymphoma, brain tumors or Wilms tumor. Method: This retrospective cohort study reviewed 252 patients treated at the Children's Hospital of Eastern Ontario from 2001-2011. Patients were included if they developed clinical symptoms of CIPN such as limb paraesthesia, weakness and/or ataxia during chemotherapy and their treating neurologist or oncologist deemed that their symptoms were due to a peripheral cause. Patients were excluded if an MR imaging identified a central cause for their symptoms. Results: CIPN occurred in children at a frequency of $18.8 \%$ (29/154) for ALL; 9.4\% (3/32) for lymphoma; $17.9 \%(5 / 28)$ for Wilms tumor; and $23.7 \%$ (9/38) for brain tumor. Nerve conduction studies (NCS) were completed for 8/46 CIPN patients (all tumor types) and were abnormal in all but one patient. Among surviving CIPN patients (41/46), 93\% showed no clinical deficits at their last examination. Conclusions: The frequency of CIPN at our center (9-24\%) is less than that previously reported in adults receiving chemotherapy. Our study shows that children with CIPN have a favorable clinical outcome.

\section{P.101}

\section{Autism spectrum disorder in a term birth NICU population}

A Winkler-Schwartz (Montreal)* J Garfinkle (Montreal) MI Shevell (Montreal)

Background: Non-specific perinatal and neonatal risk factors have been associated with autism development; however exclusively term at-risk infants remain underrepresented in the literature. This study examines the incidence and neonatal risk factors for autism spectrum disorder (ASD) in term neonatal intensive care unit (NICU) survivors. Methods: Infants were selected for retrospective chart analysis from a single university-practice database of neonates admitted to the NICU and followed by paediatric neurology. Term infants ( $\geq 37$ weeks), born between 1991 and 2008 with $\geq$ two years follow-up were included. Principle outcomes were: ASD, cerebral palsy (CP), global developmental delay (GDD), and epilepsy. Results: 180 infants were selected from a database of 564 neonates. $10(5.6 \%)$ developed ASD, 52 (29\%) CP, 77 (43\%) GDD and 47 (26\%) epilepsy. Seventy-one (40\%) developed no adverse outcomes. Seven patients with ASD exhibited at least one other adverse outcome. Emergency caesarean section (OR, 4.12; 95\% CI, 1.0216.56) and foetal distress (OR, 5.81; 95\% CI, 1.20-28.27) were associated with ASD. Conclusions: In term NICU survivors, ASD occurs with greater incidence than in the general population, often with comorbidities, and is associated with non-specific neonatal risk factors. This highlights the importance of screening NICU survivors, particularly when comorbidities are present. 


\section{P.102}

Sport participation in epileptic children: a review of the literature

E Lewis (Ottawa) M Veilleux (Ottawa)* C Weisser (Ottawa) E Sell (Ottawa)

Background: Over the last few decades, a more permissive approach has been taken towards epileptic youth and participation in sports. Despite this progress, most studies show that epileptic children are less physically active than non-epileptic children. Design/Methods: A systematic review of Medline and SportDiscus using EBSCOhost interface was conducted. No limits were imposed for language, study design or publication date. Database searching identified 162 records for screening. Consensus of two reviewers was required to exclude a study. This resulted in 85 records and, ultimately, 33 records were included for review. Results: Providing seizures are reasonably controlled, most sports are acceptable to play, although some may require additional supervision. Studies have revealed that there is no increased injury risk among epileptic children who participate in contact sports. Not only does sport restriction impact a child from a psychosocial standpoint, but a sedentary lifestyle in this population presents greater health risks than sport participation itself. Conclusions: This review suggests that further studies should examine the epidemiology regarding seizure rates and triggers among young epileptic athletes within their respective competitive sports. This would enable physicians to provide future young athletes suffering from epilepsy with more precise data regarding risks and benefits.

\section{P.103}

\section{Brain injuries in children presenting with facial fractures}

\section{A Ranger (London)* A Grant (London) G Bowden (London) B Young (London) A Yazdani (London)}

Background: Increasing attention is being paid to the importance of minor brain injuries (BI) in children. With facial fractures, associated minor BI may be missed, particularly when major BI has been excluded. We identified the rate and characteristics of minor and major BI in children presenting with facial fractures. Methods: Over nine months, 100 consecutive patients presenting with facial fractures to our level-1 trauma centre were identified. A clinical questionnaire and checklist were completed from examination and chart review, to identify and compare; major, minor, and no BI. Statistical methods were utilized to determine risk factors for BI. Results: Among 27 eligible children, ranging in age from 6 to 18 years, $59.2 \%$ suffered a minor $(n=8)$ or major $(n=8)$ BI. Injuries resulted from assaults, falls, sports and motor vehicle accidents. Younger age was associated with an increased injury rate $(\mathrm{p}<0.05)$. Every facial fracture type was associated with a BI, except single orbital floor fractures. All patients with Lefort 2 or 3 fractures had $\mathrm{BI}(\mathrm{p}=0.008)$, as did patients with additional non-cranial injuries $(\mathrm{p}=0.004)$. Minor BI patients exhibited impaired delayed memory $(\mathrm{p}<0.001)$ weeks after the event. Mechanism and patient age were strong predictors of BI. Conclusions: BI are common sequelae of facial fractures in children. Vigilance and validated criteria for BI are necessary, as minor BI are particularly missed in this setting.

\section{P.104}

Epilepsy outcome in pediatric supratentorial cavernomas: a review of 26 cases

$R$ Rébillard (Montreal)* P Diadori (Montreal) A Larbrisseau (Montreal) L Crevier (Montreal) C Marcier (Montreal) L Carmant (Montreal)

Background: The prevalence of cerebral cavernomas (CC) is estimated at $0,4-0,8 \%$ in the general population, one fourth presenting in pediatric patients. Seizures are the most frequent symptom of supratentorial CC. We wanted to evaluate the epilepsy outcome of children with CC comparing surgical versus conservative therapy. Our hypothesis is that surgical resection is associated with a better outcome in seizure control. Methods: We conducted a chart review of all pediatric supratentorial CC cases treated at Sainte-Justine Hospital from 1998-2012. Results: Sixteen patients received conservative treatment and ten underwent resection. The surgical indication was acute hemorrhage in seven and refractory seizures in three children. In the surgical group, 7/10 patients experienced seizures and at last follow-up, only one $(14,3 \%)$ was on AED and still had seizures while six $(85,7 \%)$ were seizure and AED free. In the conservative treatment group, 11/16 patients experienced seizures, two $(18,2 \%)$ were still having seizures, four $(36,4 \%)$ were on AED and seizure free and five $(45,4 \%)$ were seizure and AED free. Conclusion: Our results suggest that there is no clear difference in seizure control between the surgical and conservative group. However, children who undergo surgery are more likely to be off medication.

\section{P.105}

\section{Chorioamnionitis in children with cerebral palsy}

\section{A Shevell (Montreal)* R Benini (Montreal) M Shevell (Montreal) $M$ Oskoui (Montreal)}

Background: The neuroinflammatory response to prenatal infection has been inconsistently linked to periventricular white matter injury and subsequent neurologic impairment. We aimed to investigate the association between histological chorioamnionitis and maternal and child factors in a cohort of children with cerebral palsy. Methods: We conducted a retrospective observational study on a cohort of children with cerebral palsy who were identified from a large population-based Cerebral Palsy Registry. Factors associated with histological chorioamnionitis were explored. Results: In 455 children with cerebral palsy, 54 had histologic chorioamnionitis reported on placental pathology $(11.9 \%)$, which was the most common placental pathology reported. These children were born significantly more prematurely (mean 33.0 weeks, SD 6.2, p 0.0003 ) and at a lower birth weight (mean 2171.9g, SD 1123.3, p 0.004) than the rest of the cohort. Although periventricular white matter injury and spastic diplegic type of cerebral palsy were more often reported in these children on univariate analysis, neuroimaging and cerebral palsy subtype were no longer significant once gestational age was adjusted for. Placentas with chorioamnionitis were also more likely to have a mass above the 90th percentile. Conclusions: Chorioamnionitis is associated with premature birth with no observed additional effect on cerebral palsy subtype or neuroimaging. The significance of larger placental mass in this population is unclear. 
P.108

A survey of infantile-onset facioscapulohumeral muscular dystrophy in Canada

JK Mah (Calgary)* K Selby (Vancouver) G Smith (Kingston) H Kolski (Edmonton) L McAdam (Toronto) J Vajsar (Toronto) G Yoon (Toronto) C Group (Calgary)

Background: Facioscapulohumeral dystrophy (FSHD) is caused by contraction of D4Z4 repeats on chromosome 4q. The prevalence across Canada remains undefined. Methods: CPNG members were surveyed to determine the clinical and genetic features of infantile FSHD (iFSHD). Results: 20/24 (83\%) physicians from 18 CPNG centers participated in the survey. Eleven (4 males, 7 females) individuals with genetically confirmed iFSHD were identified; their mean age was 19.5 (range 8.7-50.9) years. The mean length of D4Z4 was 22 (range 15-38) kb. Facial weakness was present in all but one case. The mean age at onset of facial, scapular, hip, and foot weakness was $1.0,5.8,6.5$, and 8.0 years respectively, except for one woman with congenital facial diplegia and relative sparing of other muscles until later. Four (36\%) became wheelchair-dependent (mean age $=17$ years); two $(18 \%)$ required non-invasive nocturnal ventilation (mean age $=23$ years), and five $(50 \%)$ had neurosensory hearing loss. The prevalence of iFSHD is estimated to be $<2 \%$ of all FSHD. Conclusions: Infantile FSHD is rare and is associated with a severe phenotype. The diagnosis may be delayed due to underrecognition of its unique features. This knowledge is critical to the development of successful clinical care models for individuals with FSHD across Canada. See table below.

Table 1 (P.108)

\begin{tabular}{|c|c|l|}
\hline $\begin{array}{c}\text { Genetically } \\
\text { confirmed cases }\end{array}$ & $\begin{array}{c}\text { Suspected } \\
\text { cases }\end{array}$ & \multicolumn{1}{|c|}{ Physician(s) \& site } \\
\hline 2 & 1 & Jean Mah, Alberta Children's Hospital, Calgary, AB \\
\hline 1 & 1 & Kathy Selby, British Columbia Children's Hospital, Vancouver, BC \\
\hline 0 & 0 & Edward Leung, Children's Hospital, Winnipeg, MB \\
\hline 0 & 0 & Joe Dooley, Izaak Walton Killam Hospital, Halifax, NS \\
\hline 2 & 0 & Garth Smith, CDC Hotel Dieu Hospital, Kingston, ON \\
\hline 0 & 0 & Mark Tarnopolsky, McMaster University, Hamilton, 0N \\
\hline 0 & 0 & Craig Campbell, London Health Sciences Centre, London, ON \\
\hline 0 & 0 & Gillian Hogan, Erinoak Kids Centre, Mississauga, ON \\
\hline 0 & 0 & $\begin{array}{l}\text { Chantal Poulin \& Maryam Oskoui, Montreal Children's Hospital, } \\
\text { Montreal, QC }\end{array}$ \\
\hline 0 & 1 & Guy D'Anjou, CHR Sainte-Justine, Montreal, QC \\
\hline 0 & 0 & $\begin{array}{l}\text { Hugh McMillan \& Anna McCormick, Children's Hospital of Eastern } \\
\text { Ontario, Ottawa, ON }\end{array}$ \\
\hline 5 & 0 & $\begin{array}{l}\text { Grace Yoon, Jiri Vajsar, \& Brenda Banwell, Hospital for Sick Children, } \\
\text { Toronto, ON }\end{array}$ \\
\hline 0 & 2 & Laura McAdam \& Doug Biggar, Bloorview Kids Rehab, Toronto, ON \\
\hline 0 & 0 & David Buckley, Janeway Child Health Center, St. John's, NL \\
\hline 1 & 0 & Hanna Kolski, Stollery Children's Hospital, Edmonton, AB \\
\hline Total = 11 & Total = 5 & Response rate = 20/24 (83\%) physicians \\
\hline & &
\end{tabular}

P.109

Epilepsy evolution in focal cortical dysplasia and tuberous sclerosis complex

TI Aljared (Montreal)* R Dudley (Montreal) M Jabiri (Montreal) J Montes (Montreal) M Shevell (Montreal) C Saint-Martin (Montreal) $P$ Wintermark (Montreal) $J$ Atkinson (Montreal) J Farmer (Montreal)

Background: FCD and TSC are the commonest malformations of cortical development causing intractable epilepsy. Natural history of epilepsy in these disorders and outcomes is not fully elucidated. Method: Ten-year retrospective chart-review of FCD and TSC cases at Montreal Children's Hospital (major referral centre for neurological disorders). We included all cases involved neurosurgery, neurology and neuroradiology. Results: Thus far, we reviewed 32 charts (24 FCD, 8 TSC). Presentation age ranged from birth to 13-years. In FCD Group, all but one had epilepsy $(75 \%$ intractable). The prevalent potential risk factors: febrile seizures and family history. One third had a developmental delay. Fifteen patients $(63 \%)$ required surgery, mainly lesionectomies. Of the six patients who had well-documented follow-up more than one year, three were completely seizure-free. The commonest pathology was FCD type 3. In TSC group, five -of eight- patients had epilepsy (4 intractable). No significant risk factors were found. Genetic testing was confirmatory in three cases only. Two patients had developmental delay. Two required surgery. Conclusion: This work helps in a better understanding of the natural history of epilepsy in these disorders, and success of medical/surgical interventions. Further work would help to address variability in these two disorders in epilepsy development and control.

\section{P.110}

Autistic regression in a toddler: a rare presentation of antiNMDAR encephalitis

$O$ Scott (Edmonton)* L Richer (Edmonton) K Forbes (Edmonton) $M$ Eliyashevska (Edmonton) HR Goez (Edmonton)

Background: Anti-NMDA receptor encephalitis has been typically associated with psychiatric changes (predominantly aggression), seizures, language dysfunction, dystonia, or dyskinesias. Methods: Case report. Results: A 33-month-old boy presented with decreased appetite, irritability, and insomnia, following an upper respiratory tract infection. Over the following month he lost previously-acquired language skills and social interest, finally becoming mute. Repetitive left hand movements and posturing appeared. Toe-walking and wring movements of the left wrist were observed on neurological exam. Laboratory investigations were normal, apart from mild elevation of ALT and AST, and increased CSF IgG index. Brain imaging was normal, and EEG revealed a non-specific background slowing with no seizures. Subsequently, CSF came back positive for NR1 anti-NMDAR antibodies. The patient was treated with Intravenous Immunoglobulins followed by a course of oral steroids, resulting in reacquisition of language and social skills, and resolution of left hand posturing. Conclusions: Our case emphasizes the significance of suspecting anti-NMDAR encephalitis as the cause of autistic regression, even in an age group where the diagnosis of autism is first made. We caution physicians to maintain a high index of suspicion, especially in cases where deterioration is rapid, or following a febrile illness. 


\section{P.111}

Acquired infantile bilateral striatal necrosis: a rare yet treatable disorder

$O$ Scott (Edmonton)* DS Hartfield (Edmonton) $R$ Tang-Wai (Edmonton) HR Goez (Edmonton)

Background: Acquired Infantile Bilateral Striatal Necrosis (IBSN), sometimes reported as post-infectious, manifests as a movement disorder and basal ganglia imaging abnormalities. A defect in biotin metabolism has been implied in its aetiology. Methods: Case report. Results: A 4-year-old boy presented with decreased level of consciousness and involuntary movements following febrile pharyngitis. Neurological examination showed reduced mentation, posturing of left extremities, and involuntary tongue movements. Deep tendon reflexes were brisk and clonus was elicited in the lower limbs. Babinski and Hoffman reflexes were positive on the left, as well as Palmomental, Glabellar and Snout reflexes. Laboratory workup was unremarkable, except for mild protein and WBC elevation in CSF. Cultures and serology were negative. Brain MRI revealed cortical edema, which subsequently subsided, and abnormal signals in the lentiform and caudate nuclei bilaterally, which became more prominent over the next few days. Patient was started on biotin, resulting in significant improvement in both clinical picture and MRI findings. Conclusions: Due to its rarity, the diagnosis of Acquired IBSN is not commonly considered. We suggest physicians consider IBSN in patients presenting with acute movement disorder following febrile infections, as timely treatment with biotin is effective and may result in rapid improvement.

\section{P.112}

Prognostication and the contribution of MRI in hypoxicischemic injury in infants

E Bell (Montreal)* L Rasmussen (Montreal) M Shevell (Montreal) J Yager (Edmonton) A Synnes (Vancouver) B Mazer (Montreal) S Miller (Toronto) A Majnemer (Montreal) N Muhajarine (Saskatoon) I Chouinard (Montreal) E Racine (Montreal)

Background: Magnetic resonance imaging (MRI) could improve prognostication in neonatal brain injury but without consensus about the value of MRI biomarkers, practice may remain inconsistent. Methods: A vignette-based survey of Canadian neonatologists and pediatric neurologists. Two clinical vignettes of neonatal hypoxicischemia assessed the: (1) usefulness of MRI biomarkers in prognostication and (2) certainty of prognosis based on MRI biomarkers. Assessment of the impact of MRI biomarkers on general practice evaluated: (1) the contribution of MRI biomarkers in practice; (2) their use in consulting with parents; (3) access to MRI in the Canadian context; and (4) confidence in inter-subjective reliability. Results: Early analysis based on a sample of $\mathrm{N}=43(16 \%$ response rate) suggests $63 \%$ of participants agree on the prediction of future disability (severe disability in vignette 1 , moderate disability in vignette 2 ) with remaining participants varying in their prognostication of the cases (from death to mild or no disability). Although $77 \%$ of participants reported applying MRI routinely in practice for cases of this nature, the timing of these examinations was varied. Other areas of variability in general practice and physician perspectives will be explored. Conclusions: New MRI measures generate clinical variability and interact with challenging ethical dilemmas.

\section{P.113}

The cost of pediatric primary headache: a retrospective review of 100 cases in a Canadian pediatric tertiary care centre

JW Evans (Ottawa)* E Lewis (Ottawa) R Gandhi (Ottawa) D Coyle (Ottawa) A Sumner (Ottawa) E Sell (Ottawa)

Objective: Compare direct costs of primary headache in a Canadian pediatric tertiary care centre before and after neurological consultation. Background: Pediatric primary headache results in a substantial number of referrals to pediatric neurologists. Wait times are prolonged as primary headache is rarely considered urgent. Methods: Retrospective chart review of patients aged $2-18$ years referred to neurology at the Children's Hospital of Eastern Ontario (CHEO) between 2004-2010. Medical data was collected prior to and following neurology consultation. Results: One hundred patient records were reviewed. Average costs of ER visits and imaging were $\$ 80.08$ and $\$ 70.55$ per patient prior to and after neurology consultation $(t=8.37, \mathrm{df}=99, \mathrm{p}<0.0005)$. Average wait time from referral to consultation was 101 days (range: 1 to 323). Patients averaged 0.41 and 0.16 visits to ER prior to and after neurology consultation, respectively ( $\mathrm{t}=2.808$, df 99, $\mathrm{p}<0.005$ ). Nonneurologists ordered significantly more $\mathrm{CT}$ scans $(\mathrm{N}=23)$ relative to neurologists $(\mathrm{N}=13 ; \mathrm{t}=1.7$, df 99, $\mathrm{p}<0.05)$; whereas neurologists ordered significantly more MRIs $(\mathrm{N}=21)$ relative to nonneurologists $(\mathrm{N}=8 ; \mathrm{t}=2.57$, df 99, $\mathrm{p}<0.01)$. Conclusion: Prolonged wait time to neurology consultation likely increases direct costs and ordering of CT scans for pediatric primary headache. Future research should examine the effect of decreasing wait times in this population.

\section{P.115}

\section{Cerebral diaschisis in neonatal arterial ischemic stroke}

J Roe (Calgary) T Rajapakse (Calgary) G De Veber (Toronto) X Wei (Calgary) A Kirton (Calgary)*

Objectives: Diffusion MRI (DWI) corticospinal changes remote from neonatal arterial ischemic stroke (NAIS) correlate with physiology and outcome. We hypothesized DWI can quantify acute alterations in other remote connected structures ("diaschisis"). Methods: Children from Calgary and SickKids Stroke Programs with unilateral NAIS, DWI $<10$ days from term birth, and Pediatric Stroke Outcome Measure $>12$ months (mos) were studied. Image J thresholding quantified diaschisis in connected structures using validated methods. Atrophy was measured volumetrically on MRI $>12$ mos (OsiriX). Primary outcome (total diaschisis, TD) was corrected for stroke volume and regionalized by structure. Associations to MRI timing and outcome were sought (nonparametric statistics). Results: Twenty neonates met criteria (55\% male). Median age at MRI was 72 hours. Diaschisis was observed in $16(80 \%)$. Thalamic diaschisis was common $(100 \%)$, followed by callosal $(50 \%)$ and striatal (15\%). Perilesional diaschisis estimates were variable. Structures manifesting acute diaschisis atrophied on follow-up imaging. TD correlated with stroke volume $(\mathrm{p}=0.001)$ but not with age at MRI. TD correlation to poor outcome $(\mathrm{p}=0.01)$ did not persist when corrected for infarct 
volume. Method reliability was good $(\rho>0.80)$. Conclusion: Diaschisis is common and measurable in NAIS. Potentially an early imaging biomarker of network injury, larger studies are required to determine clinical relevance.

\section{P.116}

Robotic quantification of proprioceptive deficits in perinatal stroke

A Kirton (Calgary)* J Yajure (Calgary) I Leah (Winnipeg) J Roe (Calgary) S Scott (Kingston) S Dukelow (Calgary)

Background: Perinatal stroke causes most hemiplegic cerebral palsy. Sensory dysfunction has been ignored and objective measurement tools are limited. Robotic technology can quantify complex sensory function in adult stroke but has not been applied to kids. Methods: Children from the Alberta Perinatal Stroke Project had MR confirmed unilateral perinatal stroke and upper extremity functional deficit. A bilateral exoskeletal robot (KINARM) tested planar upper limb movements in an augmented reality environment. Primary outcomes were 2 dimensional variability, shift, and contraction/expansion scores of a position-matching task. Clinical measures of sensory function (touch, proprioception, graphesthesia, stereognosis) were scored. Matched controls (age/gender) were tested. Results: Five children (median 14 yrs, 3 male) with perinatal stroke (3 PVI, 2 arterial) were compared to 7 controls. Stroke children demonstrated marked impairment in position matching including variability $(6.48 \pm 1.4 \mathrm{vs} 3.89 \pm 0.7 \mathrm{~cm})$ and shift $(5.05 \pm 2.2 \mathrm{vs} 2.00 \pm 1.3 \mathrm{~cm}, \mathrm{p}=0.001)$. Contraction/expansion ratios were also abnormal $(0.56 \pm 0.27 \mathrm{vs} 0.31 \pm 0.22 ; \mathrm{p}=0.09)$. Clinical sensory scores were lower but correlated poorly with robotic measures and motor function. Assessments were well tolerated with no adverse events. Conclusion: Robotic quantification of proprioception is feasible in perinatal stroke. Sensitivity and quantification appear superior to clinical exam. Disordered proprioception is an under-recognized component of disability and a novel therapeutic target.

\section{P.117}

Glial scaring after perinatal stroke: quantification and correlation to outcome

\section{SR Shinde (Calgary) X Wei (Calgary) A Kirton (Calgary)*}

Background: Perinatal stroke diseases cause most hemiparetic cerebral palsy: Neonatal Arterial Ischemic Stroke (NAIS), arterial presumed perinatal stroke (APPIS), and periventricular venous infarction (PVI). Gliosis, a reaction to brain injury, may be both a marker of timing and a barrier to novel therapies. We hypothesized that gliosis is measureable and associated with poor outcome. Methods: Children from the Alberta Perinatal Stroke Project were included with: (1) unilateral NAIS, APPIS, or PVI, (2) axial FLAIR MRI >24 months of age, and (3) >24 months follow-up (Pediatric Stroke Outcome Measure). Novel ImageJ software protocols quantifying gliosis were developed and validated. Gliosis scores (GS) corrected for infarct and brain volumes were compared across stroke types and outcomes. Results: Forty-three children were studied. GS were comparable between NAIS (438 \pm 171$)$ and APPIS $(407 \pm 373 \mathrm{p}=0.86)$ and between all arterial and PVI $(414 \pm 336$ vs. $360 \pm 540, p=0.69)$. Arterial GS was associated with good motor outcome $(\mathrm{p}=0.003)$ and good overall outcome $(\mathrm{p}=0.022)$. PVI GS was not associated with outcome. Method reliability was excellent $(\rho=0.99)$. Conclusion: MRI quantification of gliosis is feasible in perinatal stroke. Comparable gliosis in NAIS and APPIS provides indirect evidence of similar timing. The association of gliosis with good outcome warrants further study.

\section{P.118}

Ependymoma in children under 3 years of age: the Canadian experience

C Wilbur (Vancouver)* J Hukin (Vancouver) C Dunham (Vancouver) C Hawkins (Toronto) U Tabori (Toronto) A Carret (Montreal) L Lafay-Cousin (Calgary) $P$ McNeely (Halifax) D Eisenstat (Edmonton) B Wilson (Edmonton) D Johnston (Ottawa)

Background: Ependymomas represent approximately 30\% of infant brain tumors and prognosis remains poor. Consensus is lacking around management in this age group, as radiotherapy is controversial. Methods: This was a multicentre, retrospective review of children $<36$ months of age diagnosed with intracranial ependymoma. Twelve Canadian centres participated in data collection and pathology was reviewed centrally. Results: 74 children were identified: $44.6 \%$ were alive at a median follow-up (FU) of 7.8 years (range 1.3-16.6). Children with incomplete resection (IR) and gross total resection (GTR) had similar survival$40.7 \%$ (median FU 6.7 years; $1.3-16.6$ ) and $46.8 \%$ (median FU 8.8 years; 4.0-15.6) respectively. Amongst those with IR, $66.7 \%$ were alive who received radiation at diagnosis, versus $27.8 \%$ amongst those who did not. In those $<24$ months old, only $28.3 \%$ were alive (median FU 7.7 years; 1.3-15.6), versus $71.4 \%$ (median FU 7.9 years; 2.7-16.6) in the older group. The majority of patients $<24$ months had an additional high risk factor. Patients aged 24-36 months tended to do well across all treatment groups. All 13 patients who did not receive radiation at relapse died. Conclusions: Children with an IR who did not receive radiation had poor survival. Patients $<24$ months of age had a bad prognosis. Further prospective trials are required to determine the best treatment approach.

\section{P.119}

\section{Normal verbal abilities in a child with Joubert syndrome}

\section{E Kay (Kingston)* A MacDonald (Kingston)}

Background: Joubert syndrome is a genetic disorder characterized by abnormal breathing patterns, hypotonia, ataxia, abnormal eye movements, developmental delay, and cerebellar abnormalities. Although cognitive and language disability have historically been described in Joubert syndrome, a previous study suggested that motor and speech delay and abnormal eye movements may make assessment of intelligence and verbal skills difficult leading to underestimation of abilities in these domains. A literature review revealed three previous case reports illustrating normal verbal abilities in Joubert syndrome: a 20-year-old female with verbal IQ = 93, three children with normal language, and two adult brothers with normal, although slow, reading, writing, and speech. Case: Our patient presented at age 3 with characteristic clinical findings of hypotonia, ataxia, abnormal eye movements and developmental delay, as well as the typical "molar tooth sign" on cerebellar MRI. Although delayed speech was part of her initial presentation she was found to have verbal abilities well within the normal range on psychometric testing at age 10 . Gross and fine motor domains 
showed significant disability. Conclusions: This case further illustrates the variability in verbal abilities in Joubert syndrome and the need for testing methods and educational tools that compensate for the associated motor difficulties.

\section{P.120}

Syringomyelia in scoliotic children: rare and under estimated etiology

AA Al Jishi (Montreal)* J Farmer (Montreal)

Introduction: Sytingomyelia is relatively a common referral disease to neurosurgery clinics. The majority of the patient presents with scoliotic deformity and other associated motor or sensory dysfunction. They often correlated to tethered cord or Chiari malformation. Other rare etiologies are seldom reported in which patients might regrettably be mismanaged. Methodology: A 13-yearold girl presented with a two-year history of syringomyelia-related scoliotic deformity. She was operated for a presumptive diagnosis of Chiari malformation at another hospital. She was referred to us with progressive post operative weakness. The initial MRI at our hospital showed a holocord hemorrhagic syrinx with different ages of blood. Result: Adjusted MRI sequences for her scoliotic spine revealed an intramedullary cavernoma at the apex of her scoliosis. No associated vascular malformation was seen in catheter spinal angiogram. The lesion was resected safely with no post operative complications. Early post operative follow-up showed good gain of motor function with stable scoliosis. Conclusion: Surgeons should be vigilant toward unusual presentations in scolitic children. Such cases perhaps can benefit from a multidisciplinary team approach in which an accurate diagnosis is established and proper treatment can be planned in order to facilitate patient's functional outcome. 
Abualela, Hanadi .............P.008

Agnihotri, Sameer ...........P.029 Ahmed, Syed Uzair I.08, P.041 Ahn, Younghee................P.075 Ai, Jinglu .................046, P.051 Akl, Elie A...........................09

Al Hinai, Qasim ....G.04, P.023 Al Jishi, Ahmed A..F.05, P.120 Al-Najjar, Mohammad .....G.01 Algird, Almunder .............F.09, I.02, P.083

Alharbi, Mohammed .......P.015 Aljared, Tariq I................P.109 Almekhlafi, Mohammed A...... D.01, D.02, D.03, D.04, D.05, D.08, P.052

Almenawer, Saleh A. .......G.11

Alnaami, Ibrahim M................ P.054, P.094

Alobaid, Abdullah O.. $\mathrm{P} 083, \mathrm{P} 084$

AlQassmi, Amal A.................... .P.009, P.070

Alrashidi, Saad M. ...........P.048

Alshafai, Nabeel S. .........P.013

Alturki, Abdulahman ......P.058

Alturkustani, Murad ........P.071

Alzhrani, Gmaan ..............P.066

Andrade, Andrea V............. 04

Ang, Lee C..........................071

Apel, Sabrina .......................09

Arcaro, Justin A. ...............P.006

Aref, Mohammad H................. .E.05, F.09

Armstrong, Melissa J.......H.06

Ashworth, Nigel L. ..........P.035

Atkins , Edward J.............P.034

Atkinson, Jeffrey .............P.109

Audet, Marie-Ève.............P.047

Avery, Roger ........P.024, P.086

Azad, Meghan .....................04

Bader, Ahmad A ...............P.099

Baker, Andrew .....P.043, P.049

Banfield, Laura .................. 01

Barber, Evan .............08, P.041

Bari, Muhammad E..........P.063

Barnard, Mollie ..................H.01

Barresi, Rita .......................03

Basamh, Mohammed ........F.03

Bata, Adil ............... B.01, P.053

Baweja, Reena.....................05

Beadon, Katie E..............P.038

Beaulieu, Chandree .H.11, J.07

Bell, Emily ..........................112

Bell, Joshua D...................055

Bello-Espinosa, Luis .........J.10

Benini, Ruba ......................105

Bennardo, Michael R. E.05, F.09, P.084

Bernstein, Mark ....G.10, P.087

Bhan, Virender ...P.082
Bhandari, Mohit .....C.01, G.11

Bhanot, Kunal .....................11

Bhatnagar, Neera................ 01

Bhayana, Shelly ..............P.023

Bing, Fabrice ........................09

Bingaman, William ...........H.05

Bishay, Kirles....................H.07

Bishay, Peter ......................07

Bland, Jeremy .....................035

Blough, Michael ...............G.01

Blume, Warren T. ............P.008

Bojanowski, Michel W. P.057, P.091

Bok, Levinus ....................... 01

Bosma, Rachael..................04

Bouffet, Eric ......................06

Boulton, Mel ...................P.050

Bourque, Pierre ......F.08, P.018

Bouthillier, Alain ..............H.02

Bowden, Gregory .............P.103

Bowes, Lynette..................P.024

Boycott, Kym...................03, ..H.11, J.07, P.018

Brandman, David ............E.02, .....E.10, E.11, G.07, G.09

Brathwaite, Shakira .......P.051

Brien, Susan ....................G.09

Brisson, Mélanie ..............P.065

Brooks, Brian ......................10

Buckley, David.................P.086

Bulacio, Juan........................05

Bulman, Dennis ...............H.11,

J.07, P.018

Burneo, Jorge .......P.009, P.070

Burrell, Kelly .......P.026, P.029

Burton , Jodie ........G.04, P.023

Busby, Mark........................03

Cadotte, David W................04

Cairncross, J. G.................G.01

Campbell, Trudy ..............P.082

Carlson, Helen....................... 10

Carmant, Lionel ................P.104

Caron, Jean-Louis .................06

Carret, Anne-Sophie.........P.118

Casha, S ..........................P.093

Casserly, Courtney S.......P.012

Cenic, Aleksa ..................P.084

Chakraborty, Pranesh ........J.07

Champoux , Marie-Claude .....

.P. 058

Chan, Jennifer .....................01

Chapman, Kristine ...................

P.037, P.038

Chau, Vann ............................. 03

Chen, Robert ....................... 02

Chertkow, Howard ............H.08

Chesnelong, Charles .........G.01

Cheung, Christina ..........P.007

Chinnery, Patrick F. ..........A.03

Chodirker, Bernard ........... 04 ,

.P.097
Chou, Kelvin ......................H.06

Chouinard, Isabelle .........P.112

Chow, Michael M. ..........P.090

Christie, Sean ..................E.02, E.10, E.11, P.048

Chu, Joseph Y....................11

Chuback, Jennifer E.........G.11

Claassen, Jans ....................H.04

Clarke, David B.....G.07, G.09

Clarkin, Chantalle ............P.011

Cochrane, Douglas .............J.08

Connolly, Mary B.....J.03, J.11

Connors, Robert ................H.04

Consortium, Forge ............H.11

Corenblum, Bernard ......P.023

Costello, Fiona ......G.04, P.023

Coupland, Stuart ................F.08

Couturier, Céline ................058

Coyle, Doug .......................113

Cresswell, Silke ..............P.037

Crevier, Louis .......................09

Crevier, Louis ................P.104

Cusimano, Machael .........P.013

D'Abbondanza, Josephine A... P.046, P.051

da Costa, Leodante d. ......D.10

Dakson, Ayoub ...................02,

E.10, E.11

Damji, Omar ...C.02, J.05, J.09

Darsaut, Tim E...........01, I.04, ...I.09, I.10, I.11

Dash, Dianne ....................P.004

de Guise , Elaine ...........P.058

De Veber, Gabrielle ..........P.115

Del Bigio, Marc .................H.10

Demchuk, Andrew M.............. ....D.01, D.02, D.03, D.04, ....D.05, D.07, D.08, P.052

Demos, Michelle K.......... 03 ,

(1)

Denovan-Wright, Eileen M. .... ..P.019

deRibaupierre, Sandrine ..G.09

Desai, Jamsheed ............D.01, ....D.02, D.03, D.05, P.052

Desjardins, Monique ......P.058

deVeber, Gabrielle ..............J.04

Di Ieva, Antonio D. ..........G.03

Diadori, Paola ..................P.104

Doja, Asif ............................011

Dowlatshahi, Dariush......P.042

Dowling, Michael .............J.06

Dubé, Mireille ...................P.065

Dudley , Roy ....................P.109

Duff-Canning, Sarah .........H.06

Dukelow, Sean .................P.116

Dunham, Christopher ......P.118

Dunn, Sandra ......................06

Dupré, Nicolas .................P.003

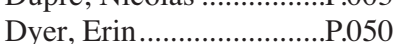

Dyment, David ..................H.11
Ebrahim, Shanil ......C.01, E.09

Edlow, Jonathan A. ...........H.01

Eesa, Muneer ...................D.01, D.02, D.04, D.07, .D. 08, P.052

Eisenstat, David ...............P.118

Elayoubi, Karim .....I.06, P.057

Eliyashevska, Myroslava P.110

Elliott, Cameron A............F.02

Elliott, Hannah R..............A.03

Emsley, Hedley C. ............A.03

Engelbrecht, Andre .........P.096

Eslinger, Paul ......................06

Estabrooks, Lorna ....F.10, F.11

Evans, Jeanette .......J.02, P.113

Everett, Chris ......................03

Evoy, François .................P.073

Falenchuk, Olesya............P.013

Fallah, Aria ............. .01, E.09,

......................................056

Farmer, Jean-Pierre

P.109, P.120

Farooq, Faraz ....................018

Farrokhyar, Forough .... E.05, F.09, P.056

Farrugia, Maria E................03

Fehlings, Michael G. ............... B. 04, E.09

Ferguson, Leslie W. ... P. 021, P.077

Fernandez, Bridget .I.07, P.096

Feyz, Mitra ........................058

Fierstra, Jorn ....................D.10

Findlay, Max ............. .03, I.11

Fiorella, David ................P.040

Fisher, Joseph A................D.10

Fitzpatrick, Wendi ............P.074

Foltz, Warren D..................051

Foote, Clary J.......................09

Forbes, Karen ....................P.110

FORGE Canada Consortium,

The .......................................... 07

Fourney, Daryl .................. 01, ...E.03, E.04, E.08

Fox, Susan............................06

Friedman, Jan M. .................03

Fryer, Alan ....................... C.03

Gagné Brosseau, Marie-Sarah.

P073

Gallagher, Clare M. ..........F.11

Gandhi, Rohit .........J.02, P.113

Ganesh, Aravind....H.09, P.014

Gano, Dawn ..........................03

Garfinkle, Jarred .............P.101

Gentili, Fred .....................P.028

Geraghty, Michael T.

.H.11, J.07

Gervais, Charles B..... ..D.09, P.005, P.078

Geschwind, Michael .......P.001

Gevry, Guylaine

.I.09 
Giacobbe, Peter ...................02

Gibson, Gillian .................P.037

Gill, David ..........................06

Gillis, Christopher C. .......... 08

Glass, Hannah C....................03

Goez, Helly R.......P.110, P.111

Gonzalez-Martinez, Jorge A....

........................................05

Gooderham, Peter A........P.038

Gospe, Sidney ..................A.01

Gosselin-Lefebvre, Stéphanie.. .P.001, P.002

Gosselin, Sylvie ...............P.073

Gould, Peter .....................P.001

Goyal, Mayank ...............D.01, .....D.02, D .03, D .04, D .05, ....D.06, D.07, D.08, P.052

Grant, Aaron.........P.062, P.103

Green, David E................051

Griffin, Helen .......................03

Group, Canadian Pediatric

Neuromuscular .................P.108

Guilbert, François .................06

Guillemette, Annie ..........P.001

Gumin, Joy ......................P.029

Gururangan, Sridharan .....G.06

Guyatt, Gordon H. .............01

Hader, Walter ........................11

Haghir , Edris ....................045

Haji, Faizal A........G.07, G.09

Hamani, Clement ..............B.02

Hamilton, Mark G....F.10, F.11

Hammond, Robert R.......P.007

Han, Sang Myung ............P.051

Hardy, Steven A.................03

Hartfield, Dawn S. ..........P.111

Hartmann, Hans ..............A.01

Hawkins, Cynthia..G.06, P.118

Hayman-Abello, Brent....P.007

Hebert-Blouin, Marie-Noelle...

.P.092

Hernandez-Ronquillo, Lizbeth H.03, P.004

Hnenny, Luke ......................01

Hogan, Thomas G. ..........P.096

Holliman, Damian ............P.028

Horvath, Gabriella ...C.03, J.11

Horvath, Rita......................03

Hosier, Gregory W. P.019, P.020

Houlden, David ........D.10, F.08

Huang, Lijia .....................P.018

Huang, Xue-yin................P.068

Huang, Yuexi ......................02

Hudson, Judith ....................03

Hukin, Juliette ..................P.118

Hunter, Gary ...................... 03 , P.005, P.034, P.045

Hurlbert, R. J.....................093

Husak, Sharon ...................094

Hynynen, Kullervo ..............02

Ibrahim, George M................. .C.01, E.09, I.05
Ichord, Rebecca ...................06

Ikeda, Kristin .........H.04, P.098

Irani, Sarosh R...............P.001

Ivanishvili, Zurab ................04

Ja Ja, Blessing ..................P.044

Jabado, Nada ......................06

Jabiri, M............................109

Jack, Andrew B.03, I.01, P.090

Jacob, Jacob ...........................07

Jacobson, Dustin ...............G.11

Jacova, Patrizio ................P.072

Jacques, Line ........P.067, P.092

Jagadeesh, Harshita .........P.087

Jaggumantri, Sravan ........A.01

Jahangirvand, Alex ....

..P.034, P.045, P.074, P.076

Jalali, Shahrzad ....P.029, P.031

James, Matthew T. .............F.01

Jette, Nathalie ......................0.01

Jia, Xiao-xiong ...................068

Jichici, Draga ..........I.02, P.056

Johnston, Dean .................P.038

Johnston, Donna ...P.100, P.118

Jones, Michelle-Lee... P.008, P.009, P.070

Jones, Steven J....................03

Kachur, Edward .................084

Kalra, Sanjay.......................07

Karunanayake, Chandima........ .P.069

Kassam, Amin ........F.08, P.042

Kaufmann, Anthony..........H.10

Kay , Emily .....................P.119

Kelly, Michael E. ...............08, P.040, P.041, P.095

Kennedy, Nancy .................H.06

Kerr, Richard ........................01

Khan, Osaama H. .P.027, P.028

Khan, Salman A..............P.063

Khezri, Navid .......................044

Kia, Maryam ........................07

Kindrachuk, Danica...........E.08

Kirk, Andrew .....................069

Kirschner, Aaron ................062

Kirton, Adam ..........C.02, J.05, ........J.06, J.09, J.10, P.115, P.116, P.117

Kletke, Stephanie N. .......P.083

Klironomos, George.........P.028

Kolski, Hanna ...................P.108

Kongkham, Paul..............P.027

Korshunov, Andrey ..........G.06

Koshly, Dilip ..............10, F.11

Kotsovsky, Oleg ......C.02, J.09

Kudo, Gen ..........................051

Kulkarni, Abhaya V..........C.01

Kwiatkowski, Brenda ......D.09

Labidi, Moujahed .P.065, P.091

Lafay-Cousin, Lucie .......P.118

Laforce, Robert J. ............P.001

Lam, Susy .........................D.11

Lamoureux , Julie ..........P.058

Lancu-Gontard, Dana .........I.06
Lang, Anthony ......H.06, P.015

Lang, Frederick F...........P.029

Lapointe, Geneviève .......P.091

Larbrisseau, Albert .........P.104

Lasry, Oliver J......P.059, P.064

Lau, Jonathan .....................033

Lavoie, Pascale ................P.091

Leah, Ion ...........................116

LeBlanc , Joanne .............P.058

Leduc, Karine...................P.003

Lee, Donald H.....P.007, P.050

Lee, Esther ...........................097

Lee, Suzi................................. 07

Lehman, Anna...............01, J.11

Leung, Stan L..................P.006

Levine, Mitchell ...............P.043

Lewis, Evan .J.02, P.102, P.113

Liang, Jianxiang ................. 01

Liang, Shuli ......................... 01

Lillquist, Yolanda ................. 11

Lim, Jordan ........................044

Lipsman, Nir A.02, B.02, G.10

Litvan, Irene .........................06

Liu, Victor ............................08

Lo, Benjamin W.............P.043, .P.044, P.049

Lochmuller, Hanns..............03

Louffat-Olivares, Ada .....P.044

Louppe, Jean- Marie .......P.067

Lownie, Steve ..................P.050

Lowry, Noel .....................P.004

Lozano, Andres M..A.02, B.02

Luchman, H. A....................01

Luu, Judy ...........................F.01

Ma, Hui ..............................068

MacDonald, Athen ...........P.119

Macdonald, R. Loch.........I.05, .P.043, P.044, P.046,

..P.049, P.051, P.055, P.056

MacKenzie, Alex ...............018

MacKenzie, Mark ................02

Madhani, Jamila ..............P.037

Magun, Rakesh R............P.079

Mah, Jean K...................P.108

Mai, Lauren M................P.012

Majewski, Jacek ......H.11, J.07

Majnemer, Annette ..........P.112

Makoyeva, Alina ..................09

Maleki, Mohammad.........F.03, ...F.07, P.058, P.060, P.061

Maloney, William............P.053

Mansouri, Alireza ...C.01, E.09

Mapstone, Mark ................H.06

Marcadier, Janet .................H.11

Marcier, Claude...............P.104

Marcoux, Judith ................F.03, ....F.04, F.07, P.058, P.059,

..P.060, P.061, P.064, P.067

Maroun, Falah B. .............I.07, P.024, P.086, P.096

Marras, Connie......H.06, P.015

Marshall, Fred ...................H.06

Martel, Philippe....P.060, P.061
Martin, Charmaine .............. 02

Masino, Susan A................075

Masiowski, A P. ...............076

Mason, Warren P..............P.027

Masood, Shaheen ..............C.04

Mast, Benjamin .................H.06

Matte, Marie ..........G.07, G.09

Maxner, Charles ...............P.082

Mazer, Barbara ..................P.112

McAdam, Laura ..............P.108

McCredie, Victoria A................ .P.044, P.049

McDonell, Laura ...............H.11

McLachlan, Richard S............. P.007, P.009, P.070

McMillan, Hugh .....J.07, P.100

McNeely, P. Daniel .........P.118

Meaney, Christopher ........H.06

Mechor, Brad ........G.04, P.023

Meguro, Kotoo .....................095

Megyesi, Joseph ................033

Mendelsohn, Danny ..........G.10

Menon, Bijoy ...................D.01, ....D.02, D.04, D.06, D.07, D. 08, P.052

Metz, Luanne .......................09

Mikulis, David J....B.04, P.015

Miller, Steven P. .....J.03, P.112

Mineyko, Aleksandra .........J.10

Ming, Alec ........................A.03

Mirsattari, Seyed M. H.04, P.006, P.007, .P.009, P.070

Mishra, Sachin ................D.01, ....D.02, D.03, D .05, P.052

Modi, Jayesh ..........D.04, D.08

Moharir, Mahendranath ..P.011

Moien Afshari, Farzad ....H.01, H.03, P.004, P.005

Monsalves, Eric ...............P.032

Montes, Jose.....................P.109

Morgan, Debra G............P.069

Morrish, William...............D.01

Morrow, Sarah A.............P.081

Muhajarine, Nazeem .......P.112

Muhammad, Waqas S. ...P.063

Multani, JK .........................084

Murphy, Tyler........................11

Murray, Gerald ....................07

Najm, Imad ..........................05

Nambiar, Vivek ..............D.01, .....D.02, D.03, D.05, P.052

Narous, Mariam ...............P.075

Nguyen, Dang K. ..H.02, P.003

Nguyen, Rita ....................P.093

Nguyen, Thanh .....................06

Nguyen, Thanh.................P.042

Nichol, Alan ........................05

Noble, Barbara ..................... 07

Norton, Jonathan .............P.094

O'Kelly, Cian J. .................03,

...F.02, P.090

Obaid, Sami .........................091 
Oskoui, Maryam ..............P.105

Owen, Richard .................P.054

Paci, Michael ..................P.059

Packer, Roger J...............G.06

Pang, Trudy D..................H.01

Parks, Natalie .....................082

Patel, Millan .........................11

Patoli, Iqra .........................063

Patrice, Finet .....................057

Patten, Scott ....................H.09

Pelz, David ..........................050

Persad, Carol .......................H.06

Petropoulos, Jo-Anne ......P.056

Pfeffer, Gerald....................03

Pfister, Stefan ......................06

Phillips, David ..................H.10

Plecko, Barbara .................A.01

Polis, Thomas ......................08

Pontifex, Carly ..................G.01

Poon, Candice ...................G.01

Poskitt, Kenneth J. ...............03

Prince, Ron...............F.10, F.11

Purser, Matthew J. ..........P.100

Qadeer, Mohsin ................P.063

Qazi, Abdul A. ..................D.07

Qazi, Emmad ...................D.07

Rabinstein, Alejandro......P.002

Racine, Eric .....................P.112

Radunovic, Aleksandar ....A.03

Rajapakse, Thilinie.J.05, P.115

Rajput, Alexander P.021, P.076

Rajput, Michele................D.09

Ramachandran, Shruthi...P.049

Raman, Ashok ...................A.03

Ramaswamy, Vijay ..........G.06

Ramesh, Aravind V..........A.03

Ramkumar, Jon ...............P.044

Ranger, Adrianna .P.062, P.103

Rasmussen, Lisa Anne ....P.112

Ravishankar, Nidhi .........P.025

Raymond, Jean..........I.01, I.03, ....I.04, I.06, I.09, I.10, I.11

Rébillard, Rose-Marie.....P.104

Reddy, Deven ........C.01, P.056

Reddy, Kesava....................05, .......E.07, F.09, G.11, P.083

Reginold, William.H.06, P.015

Remke, Marc .....................06

Rempel, Jeremy .............P.054

Rho, Jong M........................075

Richer, Lawrence ................110

Ritchie, Krista .................... 01

Ritsma, Benjamin R........P.042

Rivest, Donald ....................003

Rizek, Philippe ...............P.007

Robert, Antony .....................03

Roberts, Derek J. ..................01

Rodriguez, Amadeo ...........F.09

Roe, Jamie ................. .05, J.09,

...........................115, P.116

Ross, Colin ........................... 03

Rossetti, Andrea ..............P.002

Rothberg, Brandon ............H.06
Roy, Daniel ................03, I.04, I. I.06, I.10

Rubenfeld, Gordon ..........D.10

Rudmik, Luke .......G.04, P.023

Rutka, James T................... 01

Ryu, Won Hyung A...................

...........................04, P.023

Sahajpal, Viki ..................P.096

Sahlas, Demetrios J. ................. ..I.02, P.079

Saint-Martin, C .................109

Salazkin, Igor .......................09

Saleh, Solin .......................069

Salehi, Fateme ....................033

Salman, Michael .............. .04, P.097, P.098

Salvarinova, Ramona .......J.01, ........................................ J.11

Sankar, Tejas ....................... 02

Sargent, Michael A............... 03

Sarkozy, Anna ...................A.03

Savard, Martin ...............P.001, ..P.002, P.003, P.047, P.065

Savedia-Cayabyab, Sheila ....... .................................P.076

Sawyer, Sarah ...................H.11

Schüeller, Ulrich ..................06

Schwartz, Michael L........A.02

Schwartzentruber, Jeremy ......... H.11, J.07

Scott, Ori ..............P.110, P.111

Scott, Steve ........................116

Selby, Kathryn .........J.11, P.108

Sell, Erick ....J.02, P.102, P.113

Seres, Peter ...........................07

Serletis, Demitre ...............H.05

Shane, Hawksworth ...........F.06

Shankar, Jai J. .....................01, ……...................048, P.053

Sharma, Julia D ..................05

Shen, Yaoqing ....................03

Shevell, Allison .................P.105

Shevell, Michael I.

..P.101, P.105, P.109, P.112

Shinde, Siddharth R.

......................... .09, P.117

Shuaib, Ashfaq …...D.04, D.08

Shyr, Casper ......................... 01

Sinclair, Graham .............A.01, J.01, J.11

Sinclair, John .......................08

Singh, Sanjay K..............P.029

Singhal, Ashutosh ..............J.08

Sly, William............................ 01

Smith, Amanda ................H.11,

.........................J.07, P.018

Smith, Garth ........................108

Smith, Jickie.......................075

Snead, Carter....................... 01

Spacey, Sian D...............P.072

Spears, Julian ....................P.044

Spiess, Michael .................E.01

Stahnisch, Frank W.........P.014
Starreveld, Yves ....G.04, P.023

Steinbok, Paul ...................... 08

Stewart, Teri...........D.04, D.08

Stockler, Sylvia ....................11

Stotts, Grant ..........................042

Straub, Volker .....................03

Stroman, Patrick ................B.04

Stuckless, Teri ...................P.024

Sulman, Erik P. ..................029

Sumner, Amanda ...............P.113

Suri, Mohnish ...................... 03

Susan, Herman T.................01

Switzer, Jasmin P..............E.06

Sylvia, Stockler ........A.01, J.01

Synnes, Anne.....................P.112

Tabori, Uri .........................118

Takroni, Radwan .............P.044

Tang-Wai, David ...............H.06

Tang-Wai, Richard ...........P.111

Tariq, Asma ......................P.051

Taylor, Michael D............G.06

Teal, Philip .......................P.038

Tellez-Zenteno, Jose F. ..H.03, P.004

Teutonico, Federica ...........C.01

Thibault-Halman, Ginette ....... E.02, E.10, E.11

Thompson, Andrew E....P.071

Tjahjadi, Anindita ...........P.097

Tobias, Rose ....................P.075

Toyota, Brian .........G.05, G.08

Trudelle, Anne-Marie ......P.047

Tu, Albert D. .......................08

Turgeon, Chantal A............F.08

Turmel, André .....................091

Tymchak, Zane ....................08, ..P.040, P.095

Tymianski, Michael .........D.10

Urbaneja, Geberth ....F.10, F.11

Vaidya, Sujit........................03

Vajsar, Jiri .......................P.108

Vallance, Hilary ...................J.11

van Hove, Johan ...............A.01

van Karnebeek, Clara ...............

.........A.01, C.03, J.01, J.11

VanAdel, Brian...............P. 050

Vandorpe, Robert ................053

Vann, Chau .........................J.11

Vartanian, Alenoush ................. ...G.02, P.029

Veilleux, Marc ..................P.102

Veinot, John ....................P.018

Venance, Shannon L................. P.012, P.071

Verreault, Steve ..............P.047

Vescan, Allan ...................P.028

Vines, Douglass C...........P.051

Voll, Christopher L.

..D.09, P.077

Volny, Ondrej ..................D.01, .D.02, D.03, D.05

Vrbancic, Mirna .................004

Waheed, Abdul ..................... 01
Walling, Simon .................... 01

Walter, Stephen D............C.01

Wan, Hoyee ...........P.051, P.055

Wang, Bill H. .......P.033, P.050

Wang, Xiao-dong .............P.068

Warman Chardon, Jodi ............. ..H.11, P.018

Wasserman, Wyeth .............J.01

Watherhouse, Karen..... ..H.03, P.004

Wei, Xing Chang ..P.115, P.117

Weil, Alexander ..................091

Weill, Alain .......I.04, I.06, I.10

Weiss, Sam ...........................01

Weisser, Caroline .............P.102

White, Chris M. ..............P.078

Whiting, Sharon ...............P.011

Wiebe, Samuel ......................01

Wilbur, Colin ......................118

Wilgenbusch, Chelsea...............

.............................03, E.04

Williams, Elizabeth ............J.06

Williamson, Jackie ..........P.023

Wilson, Beverly ...............P.118

Wilson, Ian J. .....................03

Winer, John .......................... 03

Winkler-Schwartz, Alexander.. ..F.04, P.101

Wintermark, P ....................109

Wong, Peter ........................... 03

Woo, Allan .........................095

Woodside, Blake ................ 02

Woulfe, John .......................018

Wu, Adam ..........................004

Wu, Wei ...............................01

Xia, He-chu .........................068

Yager, Jerome ...................P.112

Yajure, Janice ..................P.116

Yarascavitch, Blake A.............. E.07, G.11

Yau, Ivanna .........................J.04

Yavin, Daniel ........................01

Yazdani, Arjang ....P.062, P.103

Yen Tran, Thi Phuoc .......P.003

Yip, Stephen.........................05

Yoon, Grace .....................P.108

Young, Brian .........P.062, P.103

Young, Bryan ....................H.04

Yu, Yang ..............................068

Zadeh, Gelareh................G.02, ...P.026, P.027, P.028, P.029

Zadikoff, Cindy .................H.06

Zhang, Qing-hua ..............P.068

Zhang, Yuqing ....................... 09

Zlatescu, Magdalena C. P. 071, P.081

Zoerle, Tommaso …...........051 


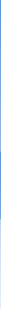

